\title{
AFFINE HIGHEST WEIGHT CATEGORIES AND AFFINE QUASIHEREDITARY ALGEBRAS
}

\author{
ALEXANDER S. KLESHCHEV
}

\begin{abstract}
Koenig and Xi introduced affine cellular algebras. Kleshchev and Loubert showed that an important class of infinite dimensional algebras, the KLR algebras $R(\Gamma)$ of finite Lie type $\Gamma$, are (graded) affine cellular; in fact, the corresponding affine cell ideals are idempotent. This additional property is reminiscent of the properties of quasihereditary algebras of Cline-Parshall-Scott in a finite dimensional situation. A fundamental result of Cline-Parshall-Scott says that a finite dimensional algebra $A$ is quasihereditary if and only if the category of finite dimensional $A$-modules is a highest weight category. On the other hand, S. Kato and Brundan-Kleshchev-McNamara proved that the category of finitely generated graded $R(\Gamma)$-modules has many features reminiscent of those of a highest weight category. The goal of this paper is to axiomatize and study the notions of an affine quasihereditary algebra and an affine highest weight category. In particular, we prove an affine analogue of the Cline-Parshall-Scott Theorem. We also develop stratified versions of these notions.
\end{abstract}

\section{INTRODUCTION}

Koenig and Xi 35 have introduced a notion of an affine cellular algebra. It is shown in 32 that an important class of infinite dimensional graded algebras, the so-called Khovanov-Lauda-Rouquier (KLR) algebras $R(\Gamma)$ of finite Lie type $\Gamma$, satisfy the graded version of the Koenig-Xi definition (see also 33 . for type $\left.\Gamma=\mathrm{A}_{\infty}\right)$. In fact, a stronger property of $R(\Gamma)$ is established in [32, namely that the affine cell ideals are idempotent. This additional property is reminiscent of the properties of quasihereditary algebras of Cline, Parshall and Scott in a finite dimensional situation, see [12].

A fundamental result of Cline, Parshall and Scott [12, Theorem 3.6] says that a finite dimensional algebra $A$ is quasihereditary if and only if the category of finite dimensional $A$-modules is a highest weight category. On the other hand, in [25] and [7] it is proved, still under the assumption that $\Gamma$ is a finite Lie type, that the category of finitely generated graded $R(\Gamma)$-modules has many features reminiscent of those of a highest weight category.

The goal of this paper is to axiomatize and study the notions of an affine quasihereditary algebra and an affine highest weight category. In particular, we prove an affine analogue of the Cline-Parshall-Scott Theorem. We also develop stratified versions of these notions.

In fact, we work in a larger generality. Let $\mathscr{B}$ be a class of Noetherian positively graded connected algebras. We introduce the notion of a $\mathscr{B}$-quasihereditary

2010 Mathematics Subject Classification. 16G99.

Research supported by the NSF grant DMS-1161094 and the Humboldt Foundation. 
algebra $H$ and a $\mathscr{B}$-highest weight category $\mathbf{C}$. Note that a $\mathscr{B}$-quasihereditary algebra $H$ does not have to be positive graded; for example, KLR algebras are not. Note also that we never assume that algebras in $\mathscr{B}$ are commutative.

If $\mathscr{B}$ consists only of the ground field $F$, then $\mathscr{B}$-quasihereditary boils down to essentially (a graded version of) the usual quasihereditary and $\mathscr{B}$-highest weight to essentially (a graded version of) the usual highest weight. If $\mathscr{B}$ is the class of all affine algebras (i.e. finitely generated positively graded commutative algebras), then we write affine quasihereditary instead of $\mathscr{B}$-quasihereditary and affine highest weight instead of $\mathscr{B}$-highest weight. Similarly, if $\mathscr{B}$ is the class of positively graded polynomial algebras, we write polynomial quasihereditary instead of $\mathscr{B}$-quasihereditary and polynomial highest weight instead of $\mathscr{B}$-highest weight, and so on.

Most known interesting examples of $\mathscr{B}$-quasihereditary algebras, including the finite Lie type KLR algebras $R(\Gamma)$, are affine and even polynomial quasihereditary. But for more general Lie types $\Gamma$ the class $\mathscr{B}$ would have to be extended. We prove in this paper that $\mathscr{B}$-quasiheredity implies finiteness of the global dimension of the corresponding algebra, provided the algebras in the class $\mathscr{B}$ have finite global dimension. It has been proved by various methods that the finite Lie type KLR algebras have finite global dimension [24, 25, [40], 7, Appendix], [32]. On the other hand, already for affine Lie types this is false.

We also develop weak versions of the notions of $\mathscr{B}$-quasihereditary and $\mathscr{B}$ highest weight. In the standard version, certain $\mathscr{B}$-modules are required to be free finite rank, while in the weak version, we only require that they are finitely generated. Moreover, we study more general notions of $\mathscr{B}$-stratified, standardly $\mathscr{B}$-stratified, and properly $\mathscr{B}$-stratified algebras and categories. These are defined for more general classes of algebras $\mathscr{B}$ and partial preorders instead of partial orders. If $\mathscr{B}$ is a class of connected algebras, then $\mathscr{B}$-quasihereditary is essentially the same as $\mathscr{B}$-properly stratified and weakly $\mathscr{B}$-quasihereditary is essentially the same as $\mathscr{B}$-stratified which is automatically $\mathscr{B}$-standardly stratified.

Module categories over KLR algebras are not the only examples of affine highest weight categories. The category of finitely generated graded modules over current algebras studied by Chari, Ion, Loktev, Pressley and many others, is another example, see 10.3 and references therein. Conjecturally, many similar categories for important positively graded Lie algebras (in characteristic 0 or $p$ ) are $\mathscr{B}$-quasihereditary for appropriate classes $\mathscr{B}$. Other examples include S. Kato's geometric extension algebras, S. Kato's categories related to Kostka systems, and Khovanov-Sazdanovich categorification of Hermite polynomials, see Section 10.

We now describe the contents of the paper in more detail. The preliminary Section 2 reviews the necessary basic material on graded algebras, modules, and categories. We introduce the notion of a Laurentian algebra - the graded algebra whose graded dimension is a Laurent series. We show that Laurentian algebras are graded semiperfect, have finite dimensional irreducible modules, and have only finitely many irreducible modules up to isomorphism and degree shift. In $\sqrt[92.3]{2}$, we review some facts about graded categories. We use $\cong$ to denote an isomorphism in a graded category and $\simeq$ to denote an isomorphism up to a degree shift.

In Section 3, we introduce Noetherian Laurentian graded abelian categories. A key example of such a category is the category $H$-mod of finitely generated graded modules over a left Noetherian Laurentian algebra $H$. In fact, a Noetherian 
Laurentian category, which has a finite set of simple objects up to isomorphism and degree shift, is always graded equivalent to $H$-mod for some left Noetherian Laurentian algebra $H$, see Theorem 3.9 .

Let $\mathbf{C}$ be a Noetherian Laurentian category and $\{L(\pi) \mid \pi \in \Pi\}$ be a complete set of simple objects in $\mathbf{C}$ up to isomorphism and degree shift. For a subset $\Sigma \subseteq \Pi$, we define a truncation functor $\mathcal{Q}^{\Sigma}: \mathbf{C} \rightarrow \mathbf{C}(\Sigma)$, where $\mathbf{C}(\Sigma)$ is the Serre subcategory consisting of all objects that belong to $\Sigma$, see 3.2 , where standard properties of the truncation factor are studied.

In Section 4, we fix a partial order ' $\leq$ ' on $\Pi$. We define the standard objects $\Delta(\pi):=\mathcal{Q}^{\Pi_{\leq \pi}}(P(\pi))$, where $P(\pi)$ is the projective cover of $L(\pi)$ for every $\pi \in \Pi$. We also define the proper standard objects $\bar{\Delta}(\pi)$, see (4.2) and compare e.g. to [16]. In $\$ 4.2$, we relate standard and proper standard modules to standardization functors following ideas of Losev and Webster [38].

Section $[5$ is devoted to the definition and fundamental properties of $\mathscr{B}$-highest weight (and $\mathscr{B}$-stratified) categories. A $\mathscr{B}$-highest weight category is defined in Definition 5.2 as a Noetherian Laurentian category such that $P(\pi)$ has filtration $P(\pi) \supset K_{0} \supset K_{1} \supset \ldots$ with $P(\pi) / K_{0} \simeq \Delta(\pi)$ and $K_{i} / K_{i+1} \simeq \Delta(\sigma)$ with $\sigma>\pi$ for all $i \geq 0$; for every $\pi \in \Pi$, the algebra $B_{\pi}:=\operatorname{End}(\Delta(\pi))^{\text {op }}$ belongs to $\mathscr{B}$; and the right $B_{\pi}$-modules $\operatorname{Hom}(P(\sigma), \Delta(\pi))$ are free finite rank for all $\pi, \sigma \in \Pi$.

In this introduction, we state the main results for $\mathscr{B}$-highest weight categories and $\mathscr{B}$-quasihereditary algebras only, ignoring the fact that many of them also hold for weakly $\mathscr{B}$-highest weight categories and weakly $\mathscr{B}$-quasihereditary algebras as well as various $\mathscr{B}$-stratified versions. The reader is referred to the body of the paper for more general results. So from now until the end of the introduction, we assume that $\mathbf{C}$ is a $\mathscr{B}$-highest weight category with respect to a partial order ' $\leq$ ' on the set $\Pi$ where $\{L(\pi) \mid \pi \in \Pi\}$ is a complete set of simples as above.

Theorem A. Let $\pi, \sigma \in \Pi$.

(i) $\operatorname{End}(L(\pi)) \cong \operatorname{End}(\bar{\Delta}(\pi)) \cong F$.

(ii) $[\bar{\Delta}(\pi): L(\pi)]_{q}=1$ and $[\Delta(\pi): L(\pi)]_{q}=\operatorname{dim}_{q} B_{\pi}$.

(iii) $\operatorname{dim}_{q} \operatorname{Hom}(P(\sigma), \bar{\Delta}(\pi))=\operatorname{rank}_{q} \operatorname{Hom}(P(\sigma), \Delta(\pi))_{B_{\pi}}$. In particular, the multiplicity $[\bar{\Delta}(\pi): L(\sigma)]_{q}$ is finite.

(iv) $\bar{\Delta}(\pi) \cong \Delta(\pi) / \Delta(\pi) N_{\pi}$, where $N_{\pi}$ is the graded Jacobson radical of $B_{\pi}$. More generally, $\Delta(\pi) N_{\pi}^{n} / \Delta(\pi) N_{\pi}^{n+1} \cong\left(\operatorname{dim}_{q} N_{\pi}^{n} / N_{\pi}^{n+1}\right) \bar{\Delta}(\pi)$. In particular, $\Delta(\pi)$ has an exhaustive filtration $\Delta(\pi) \supset V_{1} \supset V_{2} \supset$... such that $\Delta(\pi) / V_{1} \cong \bar{\Delta}(\pi)$ and each $V_{i} / V_{i+1} \simeq \bar{\Delta}(\pi), i=1,2, \ldots$, and in the graded Grothendieck group $[\mathbf{C}]_{q}$, we have $[\Delta(\pi)]=\left(\operatorname{dim}_{q} B_{\pi}\right)[\bar{\Delta}(\pi)]$.

For the proof (and strengthening of) Theorem A see Propositions 5.6 and 5.7 . Important homological properties of $\mathbf{C}$ are contained in the following theorem. For $\pi \in \Pi$ we denote $d_{\pi}:=$ gl. $\operatorname{dim} B_{\pi}$ and $d_{\Pi}:=\max \left\{d_{\pi} \mid \pi \in \Pi\right\}$. Moreover, for $\Sigma \subseteq \Pi$ we denote

$$
l(\Sigma):=\max \left\{l \mid \text { there exist elements } \sigma_{0}<\sigma_{1}<\cdots<\sigma_{l} \text { in } \Sigma\right\} .
$$

Theorem B. Let $\pi \in \Pi$ and $X$ be an object in $\mathbf{C}$.

(i) If $\operatorname{Ext}^{1}(\Delta(\pi), X) \neq 0$ then $[X: L(\sigma)]_{q} \neq 0$ for some $\sigma>\pi$. In particular, $\operatorname{Ext}^{1}(\Delta(\pi), \Delta(\sigma)) \neq 0$ implies $\pi<\sigma$.

(ii) If $\operatorname{Ext}^{i}(\bar{\Delta}(\pi), X) \neq 0$ then $[X: L(\sigma)]_{q} \neq 0$ for some $\sigma \geq \pi$. In particular, $\operatorname{Ext}^{1}(\bar{\Delta}(\pi), \bar{\Delta}(\sigma)) \neq 0$ implies $\pi \leq \sigma$. 
(iii) proj. $\operatorname{dim} \Delta(\pi) \leq l\left(\Pi_{\geq \pi}\right)$.

(iv) The global dimension of $\mathbf{C}$ is at most $2 l(\Pi)+d_{\Pi}$.

For the proof (and strengthening of) Theorem B see Lemmas 5.10, 5.17, 55.26 and Corollary 5.25 ,

Section 6 is devoted to $\mathscr{B}$-quasihereditary algerbas. Let $H$ be a left Noetherian Laurentian algebra. A (two-sided) ideal $J \subseteq H$ can be considered as a left $H$ module. The ideal $J$ is called $\mathscr{B}$-heredity if $\operatorname{Hom}_{H}(J, H / J)=0$, and as left $H$ modules we have $J \cong m(q) P(\pi)$, for some graded multiplicity $m(q) \in \mathbb{Z}\left[q, q^{-1}\right]$ and some $\pi \in \Pi$, such that $B_{\pi}:=\operatorname{End}_{H}(P(\pi))^{\text {op }}$ is an algebra in $\mathscr{B}$, and $P(\pi)$ is free finite rank as a right $B_{\pi}$-module with respect to the natural action of $B_{\pi}$ as the endomorphism algebra. The algebra $H$ is called $\mathscr{B}$-quasihereditary if there exists a finite chain of ideals $H=J_{0} \supsetneq J_{1} \supsetneq \cdots \supsetneq J_{n}=(0)$ with $J_{i} / J_{i+1}$ a $\mathscr{B}$-heredity ideal in $H / J_{i+1}$ for all $0 \leq i<n$.

Theorem C. Let $H$ be a left Noetherian Laurentian algebra. Then the category $H$-mod of finitely generated graded $H$-modules is a $\mathscr{B}$-highest weight category if and only if $H$ is a $\mathscr{B}$-quasihereditary algebra.

We refer the reader to Theorem 6.7 for a strengthening and refinement of Theorem C. Theorem $\mathrm{C}$ implies that, up to a graded equivalence, $\mathscr{B}$-highest weight categories with finite sets $\Pi$ are exactly the categories of finitely generated graded modules over $\mathscr{B}$-quasihereditary algebras. This is of course an analogue of the Cline-Parshall-Scott Theorem mentioned above.

In Section 7, we study proper costandard modules $\bar{\nabla}(\pi)$ and $\Delta$-filtrations, under the additional assumption that $\Pi_{\leq \pi}$ is finite for every $\pi \in \Pi$ (which holds in all interesting examples we know). A $\Delta$-filtration of an object $V \in \mathbf{C}$ is an exhaustive filtration $V=V_{0} \supseteq V_{1} \supseteq V_{2} \supseteq \ldots$ such that each $V_{n} / V_{n+1}$ is of the form $q^{m} \Delta(\pi)$. By Lemma 5.12(iii) for every $\pi \in \Pi$ there are only finitely many $n$ with $V_{n} / V_{n+1} \simeq \Delta(\pi)$, and the multiplicity $(V: \Delta(\pi))_{q}$ is a well-defined Laurent polynomial. We skip the precise definition of $\bar{\nabla}(\pi)$ referring the reader to $₫ 7.3$. A version of Theorem 7.13 yields key properties of the proper costandard modules:

Theorem D. Assume that $\Pi_{\leq \pi}$ is finite for every $\pi \in \Pi$. Fix $\pi, \sigma \in \Pi$. Then:

(i) The object $\bar{\nabla}(\pi) \in \mathbf{C}$ has finite length, soc $\bar{\nabla}(\pi) \cong L(\pi)$, and all composition factors of $\bar{\nabla}(\pi) /(\operatorname{soc} \bar{\nabla}(\pi))$ are of the form $L(\kappa)$ for $\kappa<\pi$.

(ii) We have

and

$$
\operatorname{dim}_{q} \operatorname{Hom}(\Delta(\sigma), \bar{\nabla}(\pi))=\delta_{\sigma, \pi}
$$

$$
\operatorname{Ext}^{1}(\Delta(\sigma), \bar{\nabla}(\pi))=0 .
$$

(iii) If $\sigma<\pi$, then

$$
\operatorname{Ext}^{1}(L(\sigma), \bar{\nabla}(\pi))=0 .
$$

(iv) If $V \in \mathbf{C}$ has a $\Delta$-filtration, then

$$
(V: \Delta(\pi))_{q}=\operatorname{dim}_{q^{-1}} \operatorname{Hom}(V, \bar{\nabla}(\pi)) .
$$

(v) (Genralized BGG Reciprocity) $(P(\pi): \Delta(\sigma))_{q}=[\bar{\nabla}(\sigma): L(\pi)]_{q^{-1}}$.

We have the (usual) useful properties of $\Delta$ - and $\bar{\nabla}$-filtrations: 
Theorem E. Assume that $\Pi$ is countable and $\Pi_{\leq \pi}$ is finite for every $\pi \in \Pi$. Let $V$ be an object of $\mathbf{C}, W$ be a direct summand of $V$, and $0 \rightarrow V^{\prime} \rightarrow V \rightarrow V^{\prime \prime} \rightarrow 0$ be a short exact sequence in $\mathbf{C}$. Then:

(i) $V$ has a $\Delta$-filtration if and only if $\operatorname{Ext}^{1}(V, \bar{\nabla}(\pi))=0$ for all $\pi \in \Pi$.

(ii) If $V$ has a $\Delta$-filtration then $\operatorname{Ext}^{i}(V, \bar{\nabla}(\pi))=0$ for all $\pi \in \Pi$ and $i>0$.

(iii) Suppose that $V \in \mathbf{C}$ has finite length. Then $V$ has $a \bar{\nabla}$-filtration if and only if $\operatorname{Ext}^{1}(\Delta(\pi), V)=0$ for all $\pi \in \Pi$.

(iv) If $V$ and $V^{\prime \prime}$ have $\Delta$-filtrations, then so does $V^{\prime}$.

(v) If $V$ and $V^{\prime}$ have finite $\bar{\nabla}$-filtrations, then so does $V^{\prime \prime}$.

(vi) If $V$ has a $\Delta$-filtration, then so does $W$.

(vii) If $V$ has a finite $\bar{\nabla}$-filtration, then so does $W$.

These results are strengthened and proved in Theorem 7.21, Lemma 7.22, Corollaries 7.23 and 7.24 .

Section 8 is devoted to a $\mathscr{B}$-analogue of Dlab-Ringel Standardization Theorem [18, Theorem 2]. We refer the reader to Theorem 8.3 for the precise statement. The idea is to axiomatize the properties of the standard modules in a graded abelian $F$-linear category $\mathbf{C}$. In this way, one gets the notion of a $\mathscr{B}$-standardizing family. Standardization Theorem then claims that given a $\mathscr{B}$ standardizing family $\Theta$, there exists a $\mathscr{B}$-quasihereditary algebra $H$, unique up to a graded Morita equivalence, such that the full subcategory category Fil $(\Theta)$ of objects in $\mathbf{C}$ with finite $\Theta$-filtrations and the category $\operatorname{Fil}(\Delta)$ of graded $H$ modules with finite $\Delta$-filtrations are graded equivalent.

In Section 9, we connect the notions of affine quasihereditary as defined in this paper and affine cellular as defined in [35. Affine cellularity assumes the existence of a 'nice' antiinvolution on an algebra, while no such assumption is made in our definition of affine quasiheredity. The main result of the section is Proposition 9.8 , which says that an affine quasihereditary algebra with a 'nice' antiinvolution $\tau$ is affine cellular.

In Section 10, we discuss examples.

Comments on the existing literature. The theory built in this paper is similar in spirit to the one developed by Mazorchuk in [39]. However, there are several crucial distinctions. Only positively graded algebras are treated in [39], which excludes our first motivating example - the KLR algebras. More general affine highest weight categories with infinite sets of simple objects are not considered in [39], which seems to exclude our other motivating example-representation theory of current algebras. The analogues of the results of Sections 3, 6, 8, 9 are not considered in [39]. On the other hand, in this paper we do not address questions related to Koszulity, which are studied in [39].

As we were preparing this article, the preprints [26] and [42] have been released. While the definitions in [26] seem to differ from ours, the theory developed there also covers one of our motivating examples, namely representation theory of current algebras. The approach of [42] is rather general but it does not seem to provide nice homological properties that we need. Another big difference is that in our picture gradings are built in and play a crucial role.

Acknowledgement. I am grateful to Steffen Koenig for many useful discussions and to Volodymyr Mazorchuk for drawing my attention to [39. 


\section{Preliminaries}

2.1. Graded algebras. By a grading we always mean a $\mathbb{Z}$-grading. Fix a ground filed $F$, and let $H$ be a graded $F$-algebra. All idempotents are assumed to be degree zero. All modules, ideals, etc. are assumed to be graded, unless otherwise stated. In particular, for a (graded) $H$-module $V, \operatorname{rad} V$ is the intersection of all maximal (graded) submodules, and soc $V$ is the sum of all irreducible (graded) submodules. We denote by $N(H)$ the (graded) Jacobson radical of $H$.

We write $q$ for both a formal variable and the upwards degree shift functor: if $V=\bigoplus_{n \in \mathbb{Z}} V_{n}$ then $q V$ has $(q V)_{n}:=V_{n-1}$. More generally, given a formal Laurent series $f(q)=\sum_{n \in \mathbb{Z}} f_{n} q^{n}$ with non-negative coefficients, $f(q) V$ denotes

$$
f(q) V:=\bigoplus_{n \in \mathbb{Z}} q^{n} V^{\oplus f_{n}} .
$$

A graded vector space $V$ is called locally finite dimensional if the dimension of each graded component $V_{n}$ is finite. Then we define the graded dimension of $V$ to be the formal series

$$
\operatorname{dim}_{q} V:=\sum_{n \in \mathbb{Z}}\left(\operatorname{dim} V_{n}\right) q^{n} .
$$

A graded vector space $V$ is called bounded below if $V_{n}=0$ for all $n \ll 0$. A graded vector space $V$ is called Laurentian if it is locally finite dimensional and bounded below. In this case $\operatorname{dim}_{q} V$ is a formal Laurent series, hence the name.

For $H$-modules $U$ and $V$, we write $\operatorname{hom}_{H}(U, V)$ for degree preserving $H$-module homomorphisms, and set $\operatorname{Hom}_{H}(U, V):=\bigoplus_{n \in \mathbb{Z}} \operatorname{Hom}_{H}(U, V)_{n}$, where

$$
\operatorname{Hom}_{H}(U, V)_{n}:=\operatorname{hom}_{H}\left(q^{n} U, V\right)=\operatorname{hom}_{H}\left(U, q^{-n} V\right) .
$$

We define $\operatorname{ext}_{H}^{d}(U, V)$ and $\operatorname{Ext}_{H}^{d}(U, V)$ similarly. If $U$ is finitely generated, then $\operatorname{Hom}_{H}(U, V)$ coincides with the set of $H$-homomorphisms from $U$ to $V$ in the ungraded category. Similar argument applies to $\operatorname{Ext}_{H}^{d}$ provided $U$ has a resolution by finitely generated projective modules, in particular if $U$ is finitely generated and $H$ is left Noetherian.

We denote by $H$-mod the category of finitely generated (graded) $H$-modules with morphisms given by hom $_{H}$. We write $\cong$ for an isomorphism in this category. For $M, N \in H$-mod, we write $M \simeq N$ to indicate that $M \cong q^{n} N$ for some $n \in \mathbb{Z}$.

2.2. Semiperfect and Laurentian algebras. Now we assume that $H$ is (graded) semiperfect, i.e. every finitely generated (graded) $H$-module has a (graded) projective cover.

Lemma 2.2. 15] The following are equivalent:

(i) $H$ is (graded) semiperfect;

(ii) $H_{0}$ is semiperfect;

(iii) $H / N(H)$ is (graded) semisimple Artinian, and idempotents lift from $H / N(H)$ to $H$

We fix a complete irredundant set of irreducible $H$-modules up to isomorphism and degree shift:

$$
\{L(\pi) \mid \pi \in \Pi\} .
$$

By the semiperfectness of $H$, the set $\Pi$ is finite. For each $\pi \in \Pi$, we also fix a projective cover $P(\pi)$ of $L(\pi)$. Let

$$
M(\pi)=\operatorname{rad} P(\pi) \quad(\pi \in \Pi),
$$


So that $P(\pi) / M(\pi) \cong L(\pi)$ for all $\pi \in \Pi$.

If $\operatorname{End}_{H}(L(\pi))$ is finite dimensional over $F$ then by the graded version of the Wedderburn-Artin Theorem [43, 2.10.10] the irreducible module $L(\pi)$ is finite dimensional. Finally, if $\operatorname{End}_{H}(L(\pi))=F$ for all $\pi \in \Pi$, then $H / N(H)$ is a finite direct product of (graded) matrix algebras over $F$ and we have

$$
{ }_{H} H=\bigoplus_{\pi \in \Pi}\left(\operatorname{dim}_{q} L(\pi)\right) P(\pi) .
$$

A graded algebra $H$ is called Laurentian if it is so as a graded vector space, i.e. locally finite dimensional and bounded below. In this case $\operatorname{dim}_{q} H$ as well as $\operatorname{dim}_{q} V$ for any finitely generated $H$-module are Laurent series.

Lemma 2.6. [31, Lemma 2.2] Let $H$ be a Laurentian algebra. Then:

(i) All irreducible $H$-modules are finite dimensional.

(ii) $H$ is semiperfect; in particular, there are only finitely many irreducible $H$-modules up to isomorphism and degree shift.

From now on, we work with a Laurentian algebra $H$. Since $H$ is semiperfect by the lemma, we can adopt the notation (2.3). We have that $H / N(H)$ is a finite direct sum of matrix algebras over division rings which are finite over $F$, in particular, $H / N(H)$ is finite dimensional.

Lemma 2.7. Let $N$ be the Jacobson radical of $H$. Then for every $m \in \mathbb{Z}$ there exists $k=k(m)$ such that $N^{k} \subseteq H_{\geq m}$.

Proof. Since there are only finitely many irreducible $H$-modules and they are all finite-dimensional, there is $n>0$ such that $H_{\geq n}$ annihilates all irreducibles, and hence $H_{\geq n} \subseteq N$, and so the two-sided ideal $H H_{\geq n} H$ generated by $H_{\geq n}$ is also contained in $N$. Since $N / H H_{\geq n} H$ is the Jacobson radical of the finite dimensional algebra $H / H H_{\geq n} H$, it is nilpotent. It follows that $N^{i} \subseteq H H_{\geq n} H$ for some $i$. Since $H$ is Laurentian, the result follows.

Laurentian algebras inherit the following pleasant property from semiprimary algebras, cf. [17, Statement 6]:

Lemma 2.8. Let $J$ be an ideal in $H$. Then $J^{2}=J$ if and only if $J=H e H$ for some idempotent $e \in H$.

Proof. The 'if-part' is clear. Conversely, suppose that $J^{2}=J$. Working in the finite dimensional algebra $\bar{H}:=H / N$, where $N$ is the Jacobson radical of $H$, we have $((J+N) / N)^{2}=(J+N) / N$. By the finite dimensional result [17, Statement $6]$, there is an idempotent $\bar{e} \in H / N$ such that $(J+N) / N=\bar{H} \bar{e} \bar{H}$. Since $H$ is semiperfect by Lemma 2.6, we have $\bar{e}=e+N$ for some idempotent $e \in H$, thanks to Lemma 2.2. Thus $J+N=H e H+N$. Then we also have for any $i$ that

$$
J+N^{i}=(J+N)^{i}=(H e H+N)^{i}=H e H+N^{i} .
$$

Making $i$ very large, looking at the degrees, and using Lemma 2.7, we now deduce that $J=H e H$.

If $V \in H$-mod, then, picking homogeneous generators $v_{1}, \ldots, v_{s}$, we have an exhaustive decreasing filtration with finite dimensional subquotients:

$$
V=H\left(v_{1}, \ldots, v_{n}\right) \supseteq H H_{\geq 1}\left(v_{1}, \ldots, v_{n}\right) \supseteq H H_{\geq 2}\left(v_{1}, \ldots, v_{n}\right) \supseteq \ldots
$$


Now take any exhaustive filtration $V=V_{0} \supseteq V_{1} \supseteq V_{2} \supseteq \ldots$ with finite dimensional subquotients. Since $V$ is bounded below, for each $m$ there exists $M$ such that $\left(V_{k} / V_{k+1}\right)_{m}=0$ for all $k>M$. So for each $n$ there exists $N$ such that $q^{n} L(\pi)$ is not a composition of $V_{k} / V_{k+1}$ for all $k>N$ and all $\pi \in \Pi$. This shows that the graded multiplicity

$$
[V: L(\pi)]_{q}:=\sum_{k \geq 0}\left[V_{k} / V_{k+1}: L(\pi)\right]_{q}
$$

is a Laurent series. The multiplicity does not depend on the choice of an exhaustive decreasing filtration with finite dimensional subquotients, since it can be described in invariant terms as follows:

$$
[V: L(\pi)]_{q}=\operatorname{dim}_{q} \operatorname{Hom}_{H}(P(\pi), V) / \operatorname{dim}_{\operatorname{end}}(L(\pi)),
$$

In particular, we can speak of composition factors of $V$. We can now embed the Grothendieck group $[H$-mod] into the free $\mathbb{Z}((q))$-module on the basis $\{[L(\pi)] \mid$ $\pi \in \Pi\}$ of classes irreducible modules, where $\mathbb{Z}((q))$ is the ring of formal Laurent series.

2.3. Graded categories. Following [8], we define a graded category as an additive category $\mathbf{C}$ equipped with an adjoint pair $\left(q, q^{-1}\right)$ of self-equivalences, called degree shift functors. For example, $H$-mod is a graded category. Just like $H$-mod, for objects $V, W$ in a graded category $\mathbf{C}$, we write $V \simeq W$ to indicate that $V \cong q^{n} M$ for some $n \in \mathbb{Z}$. Given a Laurent polynomial $f(q)=\sum_{n \in \mathbb{Z}} f_{n} q^{n}$ with non-negative coefficients and an object $V$ in $\mathbf{C}$, denote

$$
f(q) V:=\bigoplus_{n \in \mathbb{Z}} q^{n} V^{\oplus f_{n}} .
$$

We denote by hom $\mathbf{C}\left(C, C^{\prime}\right)$ the abelian group of morphisms from an object $C$ to an object $C^{\prime}$ in $\mathbf{C}$, and set:

$$
\operatorname{Hom}_{\mathbf{C}}\left(C, C^{\prime}\right):=\bigoplus_{n \in \mathbb{Z}} \operatorname{Hom}_{\mathbf{C}}\left(C, C^{\prime}\right)_{n}
$$

where

$$
\operatorname{Hom}_{\mathbf{C}}\left(C, C^{\prime}\right)_{n}:=\operatorname{hom}_{\mathbf{C}}\left(q^{n} C, C^{\prime}\right) \cong \operatorname{hom}_{\mathbf{C}}\left(C, q^{-n} C^{\prime}\right) \quad(n \in \mathbb{Z}) .
$$

In fact, this allows one to define an extended category $\hat{\mathbf{C}}$, enriched in graded abelian groups, with the same objects as $\mathbf{C}$ and morphisms given by Hom. We note that $\operatorname{End}_{\mathbf{C}}(C):=\operatorname{Hom}_{\mathbf{C}}(C, C)$ is a graded ring for any object $C$. If $\mathbf{C}$ is abelian, we define $\operatorname{Ext}_{\mathbf{C}}^{i}\left(C, C^{\prime}\right)$ similarly:

$$
\operatorname{Ext}_{\mathbf{C}}^{i}\left(C, C^{\prime}\right):=\bigoplus_{n \in \mathbb{Z}} \operatorname{Ext}_{\mathbf{C}}^{i}\left(C, C^{\prime}\right)_{n}
$$

where $\operatorname{Ext}_{\mathbf{C}}^{i}\left(C, C^{\prime}\right)_{n}:=\operatorname{ext}_{\mathbf{C}}^{i}\left(q^{n} C, C^{\prime}\right)$ for any $n \in \mathbb{Z}$.

Let $\left(\mathbf{C}, q_{\mathbf{C}}\right),\left(\mathbf{D}, q_{\mathbf{D}}\right)$ be graded categories. A graded functor from $\mathbf{C}$ to $\mathbf{D}$ is an additive functor $\mathcal{F}: \mathbf{C} \rightarrow \mathbf{D}$ equipped with a natural isomorphism $q_{\mathbf{D}} \circ$ $\mathcal{F} \stackrel{\sim}{\longrightarrow} \mathcal{F} \circ q_{\mathbf{C}}$. Using adjunctions one gets from this canonical isomorphisms $q_{\mathbf{D}}^{n} \circ \mathcal{F} \stackrel{\sim}{\longrightarrow} \mathcal{F} \circ q_{\mathbf{C}}^{n}$ for all $n \in \mathbb{Z}$. A graded functor induces a functor $\hat{\mathcal{F}}: \hat{\mathbf{C}} \rightarrow \hat{\mathbf{D}}$ on extended categories, which is equal to $\mathcal{F}$ on objects, and on morphisms it is given by compositions

$$
\hat{\mathcal{F}} f:=q_{\mathbf{D}}^{n} \mathcal{F} C \stackrel{\sim}{\longrightarrow} \mathcal{F} q_{\mathbf{C}}^{n} C \stackrel{\mathcal{F} f}{\longrightarrow} \mathcal{F} C^{\prime} \quad\left(f \in \operatorname{Hom}_{\mathbf{C}}\left(C, C^{\prime}\right)_{n}\right) .
$$


Graded categories $\mathbf{C}$ and $\mathbf{D}$ are graded equivalent, if there exists a graded equivalence between them, i.e. a graded functor, which is an equivalence of categories in the usual sense.

\section{Noetherian Laurentian CATEgories}

3.1. First properties. From now on $\mathbf{C}$ will be a graded abelian $F$-linear category. If there is no confusion, we drop the index and write hom instead of hom $\mathbf{C}$, $\mathrm{Ext}^{i}$ instead of $\mathrm{Ext}_{\mathbf{C}}^{i}$, etc. We assume that $\mathbf{C}$ has a (not necessarily finite) set

$$
\{L(\pi) \mid \pi \in \Pi\}
$$

of simple objects, which is complete and irredundant up to isomorphism and degree shift, i.e. each simple object in $\mathbf{C}$ is isomorphic to $q^{n} L(\pi)$ for exactly one pair $(n, \pi) \in \mathbb{Z} \times \Pi$. In particular,

$$
L(\pi) ¥ q^{n} L(\pi)
$$

for all $\pi \in \Pi$ and $n \neq 0$. Then $\mathbf{C}$ is called a Noetherian Laurentian category if, in addition, the following properties hold:

(NLC1) Every object $C$ in $\mathbf{C}$ is Noetherian and has a chain of subobjects $C \supseteq$ $C_{1} \supseteq C_{2} \supseteq \ldots$ such that $C / C_{m}$ is finite length and $\bigcap_{m>0} C_{m}=0$;

(NLC2) For every $\pi \in \Pi$, the simple object $L(\pi)$ has a projective cover $\varphi_{\pi}$ : $P(\pi) \rightarrow L(\pi)$

(NLC3) For all $\pi, \sigma \in \Pi$, the graded vector space $\operatorname{Hom}(P(\pi), P(\sigma))$ is Laurentian.

We denote

$$
M(\pi):=\operatorname{ker} \varphi_{\pi} \quad(\pi \in \Pi) .
$$

If $C$ is an object of $\mathbf{C}$, and $X$ is any family of morphisms from objects of $\mathbf{C}$ to $C$, we denote

$$
C X:=\sum_{f \in X} \operatorname{Im} f \subseteq C,
$$

which is well-defined since $\Delta(\pi)$ is Noetherian.

The category $H$-mod of finitely generated (graded) modules over a left Noetherian Laurentian algebra $H$ is an example of a Noetherian Laurentian category.

Lemma 3.3. Let $\mathbf{C}$ be a Noetherian Laurentian category, $\pi \in \Pi$, and $C$ be an object of $\mathbf{C}$. Then:

(i) There is an epimorphism $P \rightarrow C$, where $P$ is a finite direct sum of modules of the form $q^{n} P(\sigma)$.

(ii) The graded vector space $\operatorname{Hom}(P(\pi), C)$ is Laurentian.

(iii) Let $C \supseteq C_{1} \supseteq C_{2} \supseteq$.. be a filtration as in (NLC1). For every $m \in \mathbb{Z}$ there exists $N=N(m)$ such that $\operatorname{hom}\left(q^{m} P(\pi), C_{n}\right)=0$ for all $n \geq N$.

(iv) We have that $\operatorname{End}(L(\pi))=\operatorname{end}(L(\pi))$, and $\operatorname{dim} \operatorname{end}(L(\pi))<\infty$.

Proof. (i) It is easy to see that there is a nonzero morphism $f_{\tau}: q^{n} P(\tau) \rightarrow C$ for some $n \in \mathbb{Z}$ and $\tau \in \Pi$. If it is not an epimorphism, then there is a non-zero morphism $\bar{f}_{\sigma}: q^{m} P(\sigma) \rightarrow C / \operatorname{im} f_{\tau}$, which lifts to a morphism $f_{\sigma}: q^{m} P(\sigma) \rightarrow C$ with $\operatorname{im} f_{\tau}+\operatorname{im} f_{\sigma} \supsetneq \operatorname{im} f_{\tau}$. Continuing this way and using the assumption that $C$ is Noetherian, we get a finite family of morphisms $f_{\tau}: q^{n} P(\tau) \rightarrow C, f_{\sigma}$ : $q^{m} P(\sigma) \rightarrow C, \ldots, f_{\kappa}: q^{k} P(\kappa) \rightarrow C$ such that $\operatorname{im} f_{\tau}+\operatorname{im} f_{\sigma}+\cdots+\operatorname{im} f_{\kappa}=C$.

(ii) follows from (i) and (NLC3). 
(iii) By (ii), we have $\operatorname{dim} \operatorname{hom}\left(q^{m} P(\pi), C\right)<\infty$, and the result follows from the fact that the filtration is exhaustive.

(iv) the first statement follows from the assumption $L(\pi) \nRightarrow q^{n} L(\pi)$ for all $n \neq 0$. For the second statement, every endomorphism $L(\pi) \rightarrow L(\pi)$ lifts to a homomorphism $P(\pi) \rightarrow L(\pi)$, but $\operatorname{dim} \operatorname{hom}(P(\pi), L(\pi))$ is finite by (ii).

Corollary 3.4. Let $\mathbf{C}$ be a Noetherian Laurentian category and $C, C^{\prime}$ be object of $\mathbf{C}$. Then:

(i) There a projective resolution $\cdots \rightarrow P_{2} \rightarrow P_{1} \rightarrow P_{0} \rightarrow C$, where each $P_{i}$ is a finite direct sum of modules of the form $q^{n} P(\pi)$.

(ii) The graded vector space $\operatorname{Ext}^{i}\left(C, C^{\prime}\right)$ is Laurentian for all $i \geq 0$.

(iii) Fix $i \geq 0$. Then the vector spaces $\operatorname{Ext}^{i}(C, L(\pi))$ are finite dimensional for all $\pi \in \Pi$, and the set $\left\{\pi \in \Pi \mid \operatorname{Ext}^{i}(C, L(\pi)) \neq 0\right\}$ is finite.

Proof. (i) follows from Lemma 3.3(i), while (ii) and (iii) follow from (i) and Lemma 3.3(ii)

By Lemma 3.3(ii), for any object $C$ in $\mathbf{C}$, the graded dimension

$$
\operatorname{dim}_{q} \operatorname{Hom}(P(\pi), C)=\sum_{n \in \mathbb{Z}}\left(\operatorname{dim} \operatorname{hom}\left(q^{n} P(\pi), M\right)\right) q^{n}
$$

is a Laurent series. We define the graded multiplicity of $L(\pi)$ in $C$ to be the Laurent series

$$
[C: L(\pi)]_{q}:=\left(\operatorname{dim}_{q} \operatorname{Hom}(P(\pi), C)\right) /(\operatorname{dim} \operatorname{end}(L)),
$$

cf. Lemma 3.3(iv). We say that the multiplicity $[C: L(\pi)]_{q}$ is finite if it is a Laurent polynomial. In general, $\operatorname{dim} \operatorname{end}(L(\pi))=\operatorname{dim} \operatorname{hom}(P(\pi), L(\pi))$, so $[C: L(\pi)]_{q} \in \mathbb{Z}((q))$ (Laurent series with integral coefficients). In particular, it makes sense to speak of the composition factors of objects in $\mathbf{C}$.

Consider the $\mathbb{Z}((q))$-module which is a direct product $G(\Pi):=\prod_{\pi \in \Pi} \mathbb{Z}((q))$ of rank one free $\mathbb{Z}((q))$-modules. Write $\sum_{\pi \in \Pi} m_{\pi}[L(\pi)]$ for $\left(m_{\pi}\right)_{\pi \in \Pi} \in G(\Pi)$. For any object $C \in \mathbf{C}$, define the element

$$
[C]:=\sum_{\pi \in \Pi}[C: L(\pi)]_{q}[L(\pi)] \in G(\pi) .
$$

Denote by $[\mathbf{C}]_{q} \subseteq G(\Pi)$ the $\mathbb{Z}\left[q, q^{-1}\right]$-submodule which consists of all such $[C]$ with $C \in \mathbf{C}$. We refer to $[\mathbf{C}]_{q}$ as the graded Grothendieck group of $\mathbf{C}$.

Lemma 3.7. Let $\cdots \rightarrow C_{n+1} \rightarrow C_{n} \rightarrow C_{n-1} \rightarrow \ldots$ be a complex in $\mathbf{C}$. This complex is exact if and only if the induced complexes of vector spaces

$$
\cdots \rightarrow \operatorname{Hom}\left(P(\pi), C_{n+1}\right) \rightarrow \operatorname{Hom}\left(P(\pi), C_{n}\right) \rightarrow \operatorname{Hom}\left(P(\pi), C_{n-1}\right) \rightarrow \ldots
$$

are exact for all $\pi \in \Pi$.

Proof. The 'only-if' direction comes from the projectivity of $P(\pi)$. Conversely,

assume that $C_{n+1} \stackrel{f}{\rightarrow} C_{n} \stackrel{g}{\rightarrow} C_{n-1}$ with $\operatorname{im} f \subsetneq \operatorname{ker} g$. Let $q^{m} L(\pi)$ be a composition factor of the quotient $\operatorname{ker} g / \operatorname{im} f$. Then there is a homomorphism $\varphi \in$ $\operatorname{hom}\left(q^{m} P(\pi), C_{n}\right)$ which is in the kernel of the induced map

$$
g_{*}: \operatorname{hom}\left(q^{m} P(\pi), C_{n}\right) \rightarrow \operatorname{hom}\left(q^{m} P(\pi), C_{n-1}\right)
$$

but not in the image of $f_{*}: \operatorname{hom}\left(q^{m} P(\pi), C_{n+1}\right) \rightarrow \operatorname{hom}\left(q^{m} P(\pi), C_{n}\right)$. 
Lemma 3.8. Let $U, V$ be objects of a Noetheiran Laurentian graded category $\mathbf{C}$, $i \geq 0$ and $\operatorname{ext}^{i}(U, L)=0$ for all composition factors $L$ of $V$. Then $\operatorname{ext}_{\mathbf{C}}^{d}(U, V)=0$.

Proof. The result is proved in exactly the same way as [7, Lemma 1.1].

Theorem 3.9. Assume that $\Pi$ is finite, and set $P:=\bigoplus_{\pi \in \Pi} P(\pi)$. Then $H:=$ $\operatorname{End}(P)^{\text {op }}$ is a left Noetherian Laurentian algebra, and the functor $\operatorname{Hom}(P,-)$ is a graded equivalence of categories between $\mathbf{C}$ and $H$-mod.

Proof. This is a graded version of a standard result, cf. for example [3, Exercise on p. 55]. Note that $H$ is Laurentian by (NLC3) and left Noetherian by (NLC1). Since $P$ is projective the functor $\mathcal{F}:=\operatorname{Hom}(P,-)$ is exact.

To prove that $\mathcal{F}$ is fully faithful, note first that this is true on finite direct sums of objects of the form $q^{n} P(\pi)$. For an arbitrary object $X \in \mathbf{C}$, by Corollary [3.4(i), we have an exact sequence $P_{1}(X) \stackrel{\delta_{X}}{\longrightarrow} P_{0}(X) \stackrel{\varepsilon_{X}}{\longrightarrow} X \rightarrow 0$ in $\mathbf{C}$, with $P_{0}(X)$ and $P_{1}(X)$ finite direct sums of objects of the form $q^{n} P(\pi)$. Under $\mathcal{F}$, this exact sequence goes to a projective presentation of $\mathcal{F}(X)$. Let $f: X \rightarrow Y$ be a morphism with $\mathcal{F}(f)=0$. There exist morphisms $f_{0}, f_{1}$ which make the following diagram commutative:

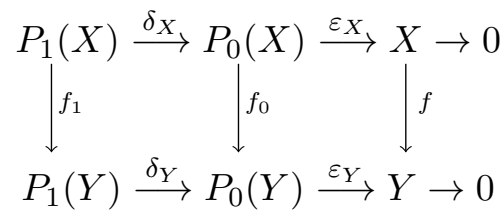

Since $\mathcal{F}(f)=0$, there is a morphism $g^{\prime}: \mathcal{F}\left(P_{0}(X)\right) \rightarrow \mathcal{F}\left(P_{1}(Y)\right)$ such that $\mathcal{F}\left(\delta_{Y}\right) \circ g^{\prime}=\mathcal{F}\left(f_{0}\right)$. However, $g^{\prime}=\mathcal{F}(g)$ for some $g: P_{0}(X) \rightarrow P_{1}(Y)$ such that $f_{0}=\delta_{Y} \circ g$. Hence $f \circ \varepsilon_{X}=\varepsilon_{Y} \circ \delta_{Y} \circ g=0$, and so $f=0$. We have proved that $\mathcal{F}$ is faithful. In order to prove that it is full, let $f^{\prime}: \mathcal{F}(X) \rightarrow \mathcal{F}(Y)$ be a morphism. We then obtain the morphisms $f_{0}^{\prime}$ and $f_{1}^{\prime}$ which make the following diagram commutative

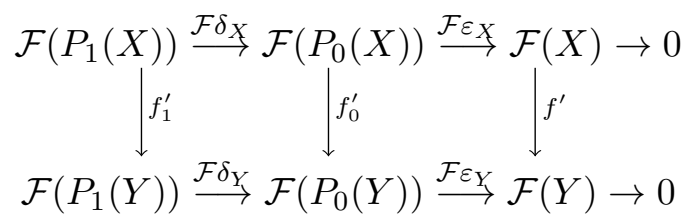

We can write $f_{0}^{\prime}=\mathcal{F}\left(f_{0}\right)$ and $f_{1}^{\prime}=\mathcal{F}\left(f_{1}\right)$, and we have $\delta_{Y} \circ f_{1}=f_{0} \circ \delta_{X}$. Since $\varepsilon_{Y} \circ f_{0} \circ \delta_{X}=0$, there is $f: X \rightarrow Y$ such that $\varepsilon_{Y} \circ f_{0}=f \circ \varepsilon_{X}$. Then

$$
\mathcal{F} f \circ \mathcal{F} \varepsilon_{X}=\mathcal{F} \varepsilon_{Y} \circ \mathcal{F} f_{0}=\mathcal{F} \varepsilon_{Y} \circ f_{0}^{\prime}=f^{\prime} \circ \mathcal{F} \varepsilon_{X} .
$$

Since $\varepsilon_{X}$ is an epimorphism, it follows that $\mathcal{F} f=f^{\prime}$.

It remains to prove that any $M \in H$-mod is isomorphic to a module of the form $\mathcal{F}(X)$ for $X \in \mathbf{C}$. Let $e_{\pi} \in H$ be the projection of $P$ to the summand $P(\pi)$. Then $e_{\pi} \in H$ is a primitive idempotent, and the modules $H e_{\pi}$ are exactly the projective indecomposable modules over $H$ up to isomorphism and degree shift. So we can use these modules to obtain a projective presentation of $M$, and then the corresponding presentation in $\mathbf{C}$ will define an object $X$ with $\mathcal{F}(X) \cong M$. 
3.2. The truncation functor $\mathcal{Q}^{\Sigma}$. We continue to use the notation of the previous subsection. In particular, $\mathbf{C}$ is a graded Noetherian Laurentian category with a complete irredundant set of simple objects $\{L(\pi) \mid \pi \in \Pi\}$ up to isomorphism and degree shift.

Let $\Sigma$ be a subset of $\Pi$. An object $X$ of $\mathbf{C}$ belongs to $\Sigma$ if any of its composition factors is isomorphic to $q^{n} L(\sigma)$ for some $n \in \mathbb{Z}$ and $\sigma \in \Sigma$. Let $\mathbf{C}(\Sigma)$ be the Serre subcategory consisting of all objects which belong to $\Sigma$.

Let $\iota_{\Sigma}: \mathbf{C}(\Sigma) \rightarrow \mathbf{C}$ be the natural inclusion, and write

$$
\mathcal{Q}^{\Sigma}: \mathbf{C} \rightarrow \mathbf{C}(\Sigma)
$$

for the left adjoint functor to $\iota_{\Sigma}$. We call $\mathcal{Q}^{\Sigma}$ a truncation functor. More explicitly, let $V \in$ C. Among all subobjects $U$ of $V$ such that $V / U$ belongs to $\Sigma$, there is a unique minimal one, which we denote by $\mathcal{O}^{\Sigma}(V)$. Using the notation (3.2), we have $\mathcal{O}^{\Sigma}(V)=V X$, where $X=\sqcup_{\pi \in \Pi \backslash \Sigma} \operatorname{Hom}(P(\pi), V)$. Then

$$
\mathcal{Q}^{\Sigma}(V)=V / \mathcal{O}^{\Sigma}(V) \text {. }
$$

Let also $\mathcal{O}_{\Sigma}(V)$ be the unique maximal subobject of $V$ which belongs to $\Sigma$.

Since $\mathcal{Q}^{\Sigma}$ is left adjoint to the exact functor $\iota_{\Sigma}$, it is right exact and sends projectives to projectives. The following is now clear:

Lemma 3.10. We have that $\{L(\sigma) \mid \sigma \in \Sigma\}$ is a complete and irredundant family of simple objects in $\mathbf{C}(\Sigma)$ up to isomorphism and degree shift. Moreover, for each $\sigma \in \Sigma$, we have that $\mathcal{Q}^{\Sigma}(P(\sigma))$ is a projective cover of $L(\sigma)$ in $\mathbf{C}(\Sigma)$. Finally, the category $\mathbf{C}(\Sigma)$ is a graded Noetherian Laurentian category.

Assume that $\Pi$ is finite, set $P:=\bigoplus_{\pi \in \Pi} P(\pi), H:=\operatorname{End}(P)^{\text {op }}$, and let

$$
\mathcal{F}_{\Pi}: \operatorname{Hom}(P,-): \mathbf{C} \rightarrow H-\bmod
$$

be the equivalence of categories from Theorem 3.9. We abuse notation and write $L(\pi)$ for $\mathcal{F}_{\Pi}(L(\pi))$ for $\pi \in \Pi$. Then $H$-mod is a graded Noetherian Laurentian category with a complete irredundant set of irreducible modules $\{L(\pi) \mid \pi \in \Pi\}$, and we can apply the above theory of the truncation functor $\mathcal{Q}^{\Sigma}$ to this situation.

Let $h \in H$ be a homogeneous element, and let $\varphi: H \rightarrow H$ be the right multiplication by $h$. Then, considering $H$ as a left regular $H$-module, we have $\varphi\left(\mathcal{O}^{\Sigma}(H)\right) \subseteq \mathcal{O}^{\Sigma}(H)$, i.e. $\mathcal{O}^{\Sigma}(H) h \subseteq \mathcal{O}^{\Sigma}(H)$, so that $\mathcal{O}^{\Sigma}(H)$ is a (two-sided) ideal of $H$. Set

$$
H(\Sigma):=H / \mathcal{O}^{\Sigma}(H) .
$$

Lemma 3.12. For $V \in H$-mod, we have $\mathcal{O}^{\Sigma}(H) V=\mathcal{O}^{\Sigma}(V)$.

Proof. This holds for $V={ }_{H} H$ and hence for free $H$-modules. Now any $V \in$ $H$-mod is a quotient of a free $H$-module, and so the result follows from the (right) exactness of $\mathcal{O}^{\Sigma}$.

By the lemma, we can regard $\mathcal{Q}^{\Sigma}(V)$ as an $H(\Sigma)$-module. In this way, $\mathcal{Q}^{\Sigma}$ becomes a functor

$$
\mathcal{Q}^{\Sigma}: H-\bmod \rightarrow H(\Sigma)-\bmod .
$$

Lemma 3.13. Let $\Pi$ be finite. With the notation as above, we have

$$
H(\Sigma) \cong \operatorname{End}\left(Q^{\Sigma}(P)\right)^{\mathrm{op}},
$$


and there is an isomorphism of functors $\mathcal{Q}^{\Sigma} \circ \mathcal{F}_{\Pi} \cong \mathcal{F}_{\Sigma} \circ \mathcal{Q}^{\Sigma}$ :

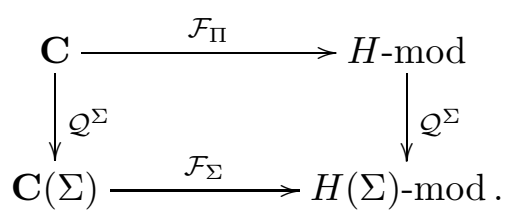

Proof. This follows from definitions and Lemmas 3.10 and 3.12 ,

\section{Standard AND PROPER STANDARD OBJeCts}

Continuing with the notation of the previous section, we now also suppose that there is a fixed surjection with finite fibers

$$
\varrho: \Pi \rightarrow \Xi
$$

for some set $\Xi$ which is endowed with a partial order ' $\leq$ '. We will usually also assume that ' $\leq$ ' is interval-finite, although this does not matter in this section. We have a preorder ' $\leq$ ' on $\Pi$ with $\pi \leq \sigma$ if and only if $\varrho(\pi) \leq \varrho(\sigma)$.

4.1. Definition of standard objects. For $\pi \in \Pi$ and $\xi \in \Xi$ we define

$$
\begin{array}{r}
\Pi_{<\pi}:=\{\sigma \in \Pi \mid \sigma<\pi\}, \Pi_{\leq \pi}:=\{\sigma \in \Pi \mid \sigma \leq \pi\}, \Pi_{\geq \pi}:=\{\sigma \in \Pi \mid \sigma \geq \pi\}, \\
\Pi_{<\xi}:=\{\sigma \in \Pi \mid \varrho(\sigma)<\xi\}, \Pi_{\leq \xi}:=\{\sigma \in \Pi \mid \varrho(\sigma) \leq \xi\},
\end{array}
$$

etc., and write $\mathcal{O}^{\leq \pi}:=\mathcal{O}^{\Pi_{\leq \pi}}, \mathcal{O}^{<\pi}:=\mathcal{O}^{\Pi_{<\pi}}, \mathcal{O}^{<\xi}:=\mathcal{O}^{\Pi_{<\xi}}, \mathcal{O}_{\leq \pi}:=\mathcal{O}_{\Pi_{\leq \pi}}$, $\mathcal{O}_{<\xi}:=\mathcal{O}_{\Pi_{<\xi}}, \mathcal{Q}^{\leq \pi}:=\mathcal{Q}^{\Pi_{\leq \pi}}, \mathbf{C}_{<\xi}:=\mathbf{C}\left(\Pi_{<\xi}\right), \mathbf{C}_{\leq \xi}:=\mathbf{C}\left(\Pi_{\leq \xi}\right)$, etc.

Recalling (3.1), we define for all $\pi \in \Pi$ :

$$
K(\pi):=\mathcal{O}^{\leq \pi}(P(\pi))=\mathcal{O}^{\leq \pi}(M(\pi)), \quad \bar{K}(\pi):=\mathcal{O}^{<\pi}(M(\pi)),
$$

and

$$
\Delta(\pi):=\mathcal{Q}^{\leq \pi}(P(\pi))=P(\pi) / K(\pi), \quad \bar{\Delta}(\pi):=P(\pi) / \bar{K}(\pi) .
$$

Note that $\bar{K}(\pi) \supseteq K(\pi)$, and so $\bar{\Delta}(\pi)$ is naturally a quotient of $\Delta(\pi)$. Moreover, head $\Delta(\pi) \cong$ head $\bar{\Delta}(\pi) \cong L(\pi)$. We call the objects $\Delta(\pi)$ standard and the objects $\bar{\Delta}(\pi)$ proper standard. By Lemma 3.10, $\Delta(\pi)$ is the projective cover of $L(\pi)$ in the category $\mathbf{C}_{\leq \pi}$. From definitions, we get:

Lemma 4.3. If $\pi \not \leq \sigma$, then

$$
\operatorname{Hom}(\Delta(\pi), \Delta(\sigma))=\operatorname{Hom}(\Delta(\pi), \bar{\Delta}(\sigma))=\operatorname{Hom}(\bar{\Delta}(\pi), \bar{\Delta}(\sigma))=0 .
$$

Lemma 4.4. For any $\pi \in \Pi$, we have

$$
\operatorname{End}(L(\pi)) \cong \operatorname{End}(\bar{\Delta}(\pi)) \cong \operatorname{Hom}(\Delta(\pi), \bar{\Delta}(\pi)) .
$$

Proof. We prove the first isomorphism, the proof of the second one is similar. Let $\theta \in \operatorname{End}(\bar{\Delta}(\pi))$. The object $\bar{\Delta}(\pi)=P(\pi) / \bar{K}(\pi)$ has unique maximal subobject $M(\pi) / \bar{K}(\pi)$, and $[M(\pi) / \bar{K}(\pi): L(\pi)]_{q}=0$. So $\theta(M(\pi) / \bar{K}(\pi)) \subseteq M(\pi) / \bar{K}(\pi)$, and $\theta$ induces an endomorphism $\bar{\theta} \in \operatorname{End}(L(\pi))$. The map $\theta \mapsto \bar{\theta}$ is injectiveindeed, if $\bar{\theta}=0$, then $\operatorname{im} \theta \subseteq M(\pi) / \bar{K}(\pi)$, whence $\theta=0$. To show that the map $\theta \mapsto \bar{\theta}$ is surjective, let $\varphi \in \operatorname{End}(L(\pi))$. Then $\varphi$ lifts to a morphism $\hat{\varphi} \in$ $\operatorname{End}(P(\pi))$. Note that $\hat{\varphi}(\bar{K}(\pi)) \subseteq \bar{K}(\pi)$, so $\hat{\varphi}$ induces a morphism $\theta \in \operatorname{End}(\bar{\Delta}(\pi))$ with $\bar{\theta}=\varphi$.

Lemma 4.5. Let $V$ be an object in $\mathbf{C}$ and $\pi \in \Pi$. Then:

(i) If $\operatorname{Ext}^{1}(\Delta(\pi), V) \neq 0$, then $V$ has a subquotient $\simeq L(\sigma)$ with $\sigma \not \leq \pi$. 
(ii) If $\operatorname{Ext}^{1}(\bar{\Delta}(\pi), V) \neq 0$, then $V$ has a subquotient $\simeq L(\sigma)$ with $\sigma \nless \pi$.

Proof. (i) follows from the fact that $\Delta(\pi)$ is the projective cover of $L(\pi)$ in the category $\mathbf{C}\left(\Pi_{\leq \pi}\right)$.

(ii) By Lemma 3.8, we may assume that $V=L(\sigma)$ for some $\sigma \in \Pi$. From the short exact sequence $0 \rightarrow \bar{K}(\pi) \rightarrow P(\pi) \rightarrow \bar{\Delta}(\pi) \rightarrow 0$ we get an exact sequence

$$
\operatorname{Hom}(\bar{K}(\pi), L(\sigma)) \rightarrow \operatorname{Ext}^{1}(\bar{\Delta}(\pi), L(\sigma)) \rightarrow 0 .
$$

If $\operatorname{Ext}^{1}(\bar{\Delta}(\pi), L(\sigma)) \neq 0$, then $\operatorname{Hom}(\bar{K}(\pi), L(\sigma)) \neq 0$, whence there is a submodule $K^{\prime} \subset \bar{K}(\pi)$ such that $\bar{K}(\pi) / K^{\prime} \simeq L(\sigma)$. So if $\sigma<\pi$, then $P(\lambda) / K^{\prime}$ belongs to $\Pi_{<\pi}$, hence $\bar{K}(\pi) \subseteq K^{\prime} \subsetneq \bar{K}(\pi)$, a contradiction.

Corollary 4.6. Let $\pi, \sigma \in \Pi(\alpha)$.

(i) If $\operatorname{Ext}^{1}(\Delta(\pi), \Delta(\sigma)) \neq 0$, then $\pi \nsupseteq \sigma$.

(ii) If $\operatorname{Ext}^{1}(\bar{\Delta}(\pi), \bar{\Delta}(\sigma)) \neq 0$, then $\pi \ngtr \sigma$.

We say that an object $V$ of $\mathbf{C}$ has a $\Delta$-filtration if there exists an exhaustive filtration $V=V_{0} \supseteq V_{1} \supseteq V_{2} \supseteq \ldots$ such that each $V_{n} / V_{n+1}$ is of the form $q^{m} \Delta(\pi)$.

Lemma 4.7. If an object $V \in \mathbf{C}$ has a $\Delta$-filtration $V=V_{0} \supseteq V_{1} \supseteq V_{2} \supseteq \ldots$, then there is an epimorphism $P \rightarrow V$, where $P$ is a finite direct sum of projectives of the form $q^{m} P(\sigma)$ such that $q^{m} \Delta(\sigma) \cong V_{r} / V_{r+1}$ for some $r$.

Proof. If $q^{m} \Delta(\sigma) \cong V_{r} / V_{r+1}$ for some $r$, then there is a non-zero morphism $f: q^{m} P(\sigma) \rightarrow V$. If $\operatorname{im} f=V$, we are done. Otherwise $V / \operatorname{im} f$ has a filtration $V / \operatorname{im} f=V_{0}^{\prime} \supseteq V_{1}^{\prime} \supseteq V_{2}^{\prime} \supseteq \ldots$ with each $V_{r}^{\prime} / V_{r+1}^{\prime}$ being a quotient of $V_{r} / V_{r+1}$. Pick $r$ with $V_{r}^{\prime} / V_{r+1}^{\prime} \neq 0$. If $V_{r} / V_{r+1} \cong q^{n} \Delta(\tau)$ there is a non-zero map $\bar{g}$ : $q^{n} P(\tau) \rightarrow V / \operatorname{im} f$, which lifts to a map $g: q^{n} P(\tau) \rightarrow V$ with $\operatorname{im} f+\operatorname{im} g \supsetneq \operatorname{im} f$. Continuing this way and using the fact that $V$ is noetherian, we get the required result.

4.2. Standardization functor. In this subsection we exploit the ideas of $\mathbf{3 8}$, $\S 2]$. Note that $\mathbf{C}_{<\xi}$ is a Serre subcategory of $\mathbf{C}_{\leq \xi}$, and the quotient category $\mathbf{C}_{\xi}:=\mathbf{C}_{\leq \xi} / \mathbf{C}_{<\xi}$ is a graded abelian $F$-linear category. In fact, $\mathbf{C}_{\xi}$ is equivalent to the full subcategory $\mathbf{C}_{\leq \xi}^{0}$ of $\mathbf{C}_{\leq \xi}$ which consists of all objects $V \in \mathbf{C}_{\leq \xi}$ with $\mathcal{O}_{<\xi}(V)=0$ and $\mathcal{O}^{<\xi}(V)=V$. It is easy to see that $\mathbf{C}_{\xi}$ satisfies the axiom (NLC1). To show that $\mathbf{C}_{\xi}$ is Noetherian Laurentian, note first that $\{L(\pi) \mid$ $\varrho(\pi)=\xi\}$ is a complete family of simple objects in $\mathbf{C}_{\xi}$ up to isomorphism and degree shift.

Lemma 4.8. Let $\varrho(\pi)=\xi$. We have that $P_{\xi}(\pi):=\Delta(\pi) / \mathcal{O}_{<\xi}(\Delta(\pi))$ is the projective cover of $L(\pi)$ in $\mathbf{C}_{\xi}$, and $\operatorname{End}_{\mathbf{C}_{\xi}}\left(P_{\xi}(\pi)\right) \cong \operatorname{End}_{\mathbf{C}_{\leq \xi}}(\Delta(\pi))$. Moreover, $\operatorname{Hom}_{\mathbf{C}_{\xi}}\left(P_{\xi}(\pi), P_{\xi}(\sigma)\right) \cong \operatorname{Hom}_{\mathbf{C}_{\leq \xi}}(\Delta(\pi), \Delta(\sigma))$ for any $\pi, \sigma \in \varrho^{-1}(\xi)$.

Proof. Working in the equivalent category $\mathbf{C}_{\leq \xi}^{0}$, we have to show that for any surjective morphism $f: M \rightarrow N$ and any morphism $g: P_{\xi}(\pi) \rightarrow N$, there is a morphism $h: P_{\xi}(\pi) \rightarrow M$ with $f \circ h=g$. Note that $f$ is surjective as a morphism in $\mathbf{C}_{\leq \xi}$, and $\Delta(\pi)$ is projective in $\mathbf{C}_{\leq \xi}$. So, if $\mathrm{p}: \Delta(\pi) \rightarrow P_{\xi}(\pi)$ is the natural surjection there is a morphism $\hat{h}: \Delta(\pi) \rightarrow M$ in $\mathbf{C}_{\leq \xi}$ such that $f \circ \hat{h}=g \circ \mathrm{p}$. Since $M \in \mathbf{C}_{\leq \xi}^{0}$, the morphism $\hat{h}$ factors to $h: \Delta(\pi) / \mathcal{O}_{<\xi}(\Delta(\pi)) \rightarrow M$.

For the second statement, note that any morphism $\theta: \Delta(\pi) \rightarrow \Delta(\sigma)$ factors to a morphism $\bar{\theta}: P_{\xi}(\pi) \rightarrow P_{\xi}(\sigma)$, and conversely any endomorphism $\bar{\theta}$ as above lifts to a morphism $\theta$ as above using the projectivity of $\Delta(\pi)$ in $\mathbf{C}_{\leq \pi}$. 
For $\xi \in \Xi$, set

$$
P_{\xi}:=\bigoplus_{\pi \in \varrho^{-1}(\xi)} P_{\xi}(\pi) \in \mathbf{C}_{\xi} \quad \Delta_{\xi}:=\bigoplus_{\pi \in \varrho^{-1}(\xi)} \Delta(\pi) \in \mathbf{C}_{\leq \xi}
$$

Corollary 4.9. Let $\xi \in \Xi$. Then $\mathbf{C}_{\xi}$ is a Noetherian Laurentian graded category graded equivalent to $B_{\xi}$-mod, where $B_{\xi}:=\operatorname{End}\left(\Delta_{\xi}\right)^{\mathrm{op}}$.

Proof. Use Lemma 4.8 and Theorem 3.9.

We have a natural exact projection functor $\mathcal{R}_{\xi}: \mathbf{C}_{\leq \xi} \rightarrow \mathbf{C}_{\xi}$. Its left adjoint

$$
\mathcal{E}_{\xi}: \mathbf{C}_{\xi} \rightarrow \mathbf{C}_{\leq \xi}
$$

is called a weak standardization functor. Moreover, a weak standardization functor is called a standardization functor if it is exact.

Lemma 4.11. Let $\varrho(\pi)=\xi$ and suppose that a weak standardization functor $\mathcal{E}_{\xi}$ exists. Then $\Delta(\pi) \cong \mathcal{E}_{\xi}\left(P_{\xi}(\pi)\right)$ and $\bar{\Delta}(\pi) \cong \mathcal{E}_{\xi}(L(\pi))$.

Proof. Since $\mathcal{E}_{\xi}$ is left adjoint to the exact functor $\mathcal{R}_{\xi}$, it sends projectives to projectives. Moreover, it is clear that the head of $\mathcal{E}_{\xi}\left(P_{\xi}(\pi)\right)$ is $L(\pi)$. Since $\Delta(\pi)$ is the projective cover of $L(\pi)$ in $\mathbf{C}_{\leq \xi}$, the first isomorphism follows. For the second isomorphism, note that the head of $\mathcal{E}_{\xi}(L(\pi))$ is $L(\pi),\left[\mathcal{E}_{\xi}(L(\pi)): L(\pi)\right]_{q}=1$, and other composition factors $L(\sigma)$ of $\mathcal{E}_{\xi}(L(\pi))$ are of the form $L(\sigma)$ for $\sigma<\pi$. So $\mathcal{E}_{\xi}(L(\pi))$ is a quotient of $\bar{\Delta}(\pi)$. Now

$$
\operatorname{Hom}_{\mathbf{C}_{\leq \xi}}\left(\mathcal{E}_{\xi}(L(\pi)), \bar{\Delta}(\pi)\right) \cong \operatorname{Hom}_{\mathbf{C}_{\xi}}\left(L(\pi), \mathcal{R}_{\xi}(\bar{\Delta}(\pi))\right)=\operatorname{Hom}_{\mathbf{C}_{\xi}}(L(\pi), L(\pi))
$$

completes the proof of the second isomorphism.

Assume now that $\Pi_{\leq \xi}$ is finite. Then by Theorem 3.9, the category $\mathbf{C}_{\leq \xi}$ is explicitly graded equivalent to a category $H_{\leq \xi}$-mod of finitely generated modules over a left Noetherian Laurentian algebra $H_{\leq \xi}$. So we will not distinguish between the two categories. Similarly we will not distinguish between $\mathbf{C}_{\xi}$ and $B_{\xi}$-mod, where $B_{\xi}=\operatorname{End}\left(\Delta_{\xi}\right)^{\mathrm{op}}$. In these terms the quotient functor $\mathcal{R}_{\xi}$ becomes the functor

$$
\mathcal{R}_{\xi}=\operatorname{Hom}_{H_{\leq \xi}}\left(\Delta_{\xi},-\right): H_{\leq \xi}-\bmod \rightarrow B_{\xi^{-}} \bmod .
$$

It always has left adjoint

$$
\mathcal{E}_{\xi}=\Delta_{\xi} \otimes_{B_{\xi}}-: B_{\xi^{-}} \bmod \rightarrow H_{\leq \xi^{-} \bmod }
$$

To summarize:

Lemma 4.12. If $\Pi_{\leq \xi}$ is finite then a weak standardization functor $\mathcal{E}_{\xi}$ exists. Moreover, $\mathcal{E}_{\xi}$ is a standardization functor if and only if $\Delta_{\xi}$ is flat as a right $B_{\xi}$-module.

\section{5. $\mathscr{B}$-Highest WEIGHT AND $\mathscr{B}$-STRATIFIED CATEGORIES}

We stick with the notation of the previous section. In particular, C is a Noetherian Laurentian category with a complete set of simple objects $\{L(\pi) \mid$ $\pi \in \Pi\}$ up to isomorphism and degree shift, $\Xi$ is a poset, and $\varrho: \Pi \rightarrow \Xi$ is as in (4.1). Recall the notation $\Delta_{\xi}=\bigoplus_{\pi \in \varrho^{-1}(\xi)} \Delta(\pi)$. From now on we assume that the partial order $\leq$ on $\Xi$ is interval finite. We also assume for simplicity that $\mathbf{C}$ is Schurian, i.e. $\operatorname{End}(L(\pi)) \cong F$ for all $\pi$. For (weakly) $\mathscr{B}$-highest weight categories this condition will hold automatically, see Proposition 5.6(i). 
The notions introduced in this section develop (and are strongly influenced by) those introduced by Cline-Parshall-Scott [12, [14] and Dlab [16].

5.1. Definition of $\mathscr{B}$-stratified and $\mathscr{B}$-highest weight categories. Let $B$ be a Noetherian Laurentian graded algebra over $F$. We say that $B$ is connected if $B_{n}=0$ for $n<0$ and $B_{0}=F \cdot 1_{B}$. If $B$ is a connected algebra, then for its Jacobson radical we have $N(B)=\bigoplus_{n>0} B_{n}$. Note that, $N(B) \supseteq N(B)^{2} \supseteq$ $N(B)^{3} \supseteq \ldots$ is an exhaustive filtration with finite dimensional quotients. Now let $V \in B$-mod. The graded dimension of the vector space $V / N V$ over $F=$ $B / N$ is called the (graded) rank of the $B$-module $V$, written $\operatorname{rank}_{q} V_{B}$. Note that if $v_{1}, \ldots, v_{r}$ is a minimal set of homogeneous $B$-generators of $V$, of degrees $d_{1}, \ldots, d_{r}$, respectively, then $\operatorname{rank}_{q} V=q^{d_{1}}+\cdots+q^{d_{r}}$. A graded polynomial algebra is a polynomial algebra $F\left[x_{1}, \ldots, x_{n}\right]$ graded by requiring that $\operatorname{deg}\left(x_{i}\right) \in \mathbb{Z}_{>0}$ for all $i=1, \ldots, n$. A (graded) affine algebra is a quotient of a graded polynomial algebra by some homogeneous ideal.

Let $\mathscr{B}$ be a fixed class of left Noetherian Laurentian algebras over $F$. For example, $\mathscr{B}$ could be the class of all left Noetherian Laurentian algebras over $F$ or a class of (graded) affine algebras, or the class of nilCoxeter algebras, etc.

Definition 5.1. The category $\mathbf{C}$ is called a $\mathscr{B}$-stratified category (with respect to $\varrho: \Pi \rightarrow \Xi)$ if the following properties hold:

(SC1) For every $\pi \in \Pi$, the object $K(\pi)$ has a $\Delta$-filtration with quotients of the form $q^{n} \Delta(\sigma)$ for $\sigma>\pi$.

(SC2) For every $\xi \in \Xi$, the algebra $B_{\xi}:=\operatorname{End}\left(\Delta_{\xi}\right)^{\text {op }}$ belongs to $\mathscr{B}$.

A $\mathscr{B}$-stratified category is called $\mathscr{B}$-properly stratified if the following properties hold:

(FGen) For every $\sigma \in \Pi$ and $\xi \in \Xi$, the right $B_{\xi}$-module $\operatorname{Hom}\left(P(\sigma), \Delta_{\xi}\right)$ is finitely generated.

(PSC) For every $\xi \in \Xi$, there is a standardization functor $\mathcal{E}_{\xi}$.

A $\mathscr{B}$-stratified category is called $\mathscr{B}$-standardly stratified if (FGen) together with the following property (SSC) hold:

(SSC) For every $\xi \in \Xi$, there is a weak standardization functor $\mathcal{E}_{\xi}$.

Definition 5.2. Let $\mathscr{B}$ be a class of connected algebras over $F$, and assume that $\Xi=\Pi$ and $\varrho=$ id. A $\mathscr{B}$-stratified category $\mathbf{C}$ is called $\mathscr{B}$-highest weight if the following property holds for all $\pi, \sigma \in \Pi$ :

(HWC) The (right) $B_{\pi}$-module $\operatorname{Hom}(P(\sigma), \Delta(\pi))$ is free finite rank.

A $\mathscr{B}$-stratified category which satisfies (FGen) is called a weak $\mathscr{B}$-highest weight category.

In the crucial case where $\mathscr{B}$ is the class of all affine algebras, we write affine highest weight instead of $\mathscr{B}$-highest weight, affine stratified instead of $\mathscr{B}$-stratified, etc. When $\mathscr{B}$ is the class of all polynomial algebras, we write polynomial highest weight instead of $\mathscr{B}$-highest weight, etc. If $\mathscr{B}=\{F\}$, then a $\mathscr{B}$-highest weight category is a graded version of the usual highest weight category [12].

Remark 5.3. Let $\xi \in \Xi$ be such that $\Pi_{\leq \xi}$ is finite.

(i) As noted in the end of 4.2 , we can identify the category $\mathbf{C}_{\leq \xi}$ with the category $H_{\leq \xi}$-mod for a left Noetherian Laurentian algebra $H_{\leq \xi}$, the category $\mathbf{C}_{\xi}$ with the category $B_{\xi}$-mod, and the quotient functor $\mathcal{R}_{\xi}$ with the functor 
$\operatorname{Hom}_{H_{\leq \xi}}\left(\Delta_{\xi},-\right): H_{\leq \xi}$-mod $\rightarrow B_{\xi}$-mod, which has left adjoint $\mathcal{E}_{\xi}=\Delta_{\xi} \otimes_{B_{\xi}}-:$ $B_{\xi}$-mod $\rightarrow H_{\leq \xi}$-mod, see Lemma 4.12. So (SSC) holds automatically.

(ii) Decompose the left regular module $H_{\leq \xi}$ as a direct sum of projective indecomposable modules $H_{\leq \xi}=\oplus_{\sigma \in \Pi_{\leq \xi}}\left(\operatorname{dim}_{q} L(\sigma)\right) P_{\leq \xi}(\sigma)$. Note using Lemma 3.10 that $P_{\leq \xi}(\sigma)=\mathcal{Q}^{\leq \xi}(P(\sigma))$, and so

$$
\begin{aligned}
\Delta_{\xi} \cong \operatorname{Hom}_{H_{\leq \xi}}\left(H_{\leq \xi}, \Delta_{\xi}\right) & \cong \bigoplus_{\sigma \in \Pi_{\leq \xi}}\left(\operatorname{dim}_{q} L(\sigma)\right) \operatorname{Hom}_{H_{\leq \xi}}\left(P_{\leq \xi}(\sigma), \Delta_{\xi}\right) \\
& \cong \bigoplus_{\sigma \in \Pi_{\leq \xi}}\left(\operatorname{dim}_{q} L(\sigma)\right) \operatorname{Hom}_{\mathbf{C}}\left(P(\sigma), \Delta_{\xi}\right) .
\end{aligned}
$$

So, if all $\Pi_{\leq \xi}$ are finite, we can restate (HWC) (resp. (FGen) as a requirement that for each $\pi$ (resp. $\xi$ ), the right $B_{\pi}$-module $\Delta_{\pi}\left(\operatorname{resp} B_{\xi}\right.$-module $\left.\Delta(\xi)\right)$ is free finite rank (resp. finitely generated). Similarly, in view of (i), we can restate (PSC) as a requirement that for each $\xi$, the right $B_{\xi}$-module $\Delta(\xi)$ is flat.

Proposition 5.5. Let $\mathbf{C}$ be a Noetherian Laurentian category, $\Xi=\Pi$, $\varrho=\mathrm{id}$, and $\mathscr{B}$ be a class of connected algebras. If $\Pi_{\leq \pi}$ is finite for every $\pi \in \Pi$, then:

(i) $\mathbf{C}$ is $\mathscr{B}$-properly stratified if and only if $\mathbf{C}$ is $\mathscr{B}$-highest weight.

(ii) $\mathbf{C}$ is $\mathscr{B}$-standardly stratified if and only if $\mathbf{C}$ is weakly $\mathscr{B}$-highest weight.

Proof. We use the notation of Remark 5.3. By part (i) of that remark, (SSC) holds automatically, so $\mathbf{C}$ is weakly $\mathscr{B}$-highest weight if and only if $\mathbf{C}$ is standardly stratified. Moreover, we can restate (HWC) as a requirement that for each $\pi \in \Pi$, the right $B_{\pi}$-module $\Delta(\pi)$ is free finite rank. So, if $\mathbf{C}$ is $\mathscr{B}$-highest weight, then the standardization functor $\mathcal{E}_{\pi}=\Delta(\pi) \otimes_{B_{\pi}}$ - is exact since $\Delta(\pi)_{B_{\pi}}$ is free and hence flat. Conversely, let $\mathbf{C}$ be a $\mathscr{B}$-properly stratified category. Then the functor $\mathcal{E}_{\pi}=\Delta(\pi) \otimes_{B_{\pi}}$ - is exact. by (PSC), hence the finitely generated module $\Delta(\pi)_{B_{\pi}}$ is flat, and hence free by a standard result, since $B_{\pi}$ is local.

5.2. Properties of $\mathscr{B}$-highest weight categories. Let $\mathbf{C}$ be a weakly $\mathscr{B}$ highest weight category as in Definition 5.2. For each $\pi \in \Pi$, we denote by $N_{\pi}$ the Jacobson radical $N\left(B_{\pi}\right)$ of $B_{\pi}$. The notation $\Delta(\pi) N_{\pi}$ is understood in the sense of (3.2).

Proposition 5.6. Let $\mathbf{C}$ be a weakly $\mathscr{B}$-highest weight category and $\pi, \sigma \in \Pi$.

(i) $\operatorname{End}(\bar{\Delta}(\pi)) \cong F \cong \operatorname{End}(L(\pi))$.

(ii) $[\Delta(\pi): L(\pi)]_{q}=\operatorname{dim}_{q} B_{\pi}$.

(iii) $\bar{\Delta}(\pi) \cong \Delta(\pi) / \Delta(\pi) N_{\pi}$.

(iv) $\operatorname{dim}_{q} \operatorname{Hom}(P(\sigma), \bar{\Delta}(\pi))=\operatorname{rank}_{q} \operatorname{Hom}(P(\sigma), \Delta(\pi))_{B_{\pi}}$. In particular, the multiplicity $[\bar{\Delta}(\pi): L(\sigma)]_{q}$ is finite.

(v) $\Delta(\pi)$ has an exhaustive filtration $\Delta(\pi) \supset V_{1} \supset V_{2} \supset \ldots$ such that $\Delta(\pi) / V_{1} \cong \bar{\Delta}(\pi)$ and each $V_{i} / V_{i+1}, i=1,2, \ldots$, is a quotient of $q^{n} \bar{\Delta}(\pi)$ for some $n \in \mathbb{Z}_{>0}$.

Proof. (i) Any endomorphism of $L(\pi)$ lifts to an endomorphism of $P(\pi)$, which in turn factors through an endomorphism of $\Delta(\pi)$. The endomorphism algebra of $\Delta(\pi)$ is positively graded and connected. We deduce that so is $\operatorname{End}(L(\pi))$. Since $\operatorname{End}(L(\pi))$ is a division ring, it follows that $\operatorname{End}(L(\pi))=F$. Now use Lemma 4.4 to complete the proof of (i). 
(ii) By (i) and (3.5), we have $[\Delta(\pi): L(\pi)]_{q}=\operatorname{dim}_{q} \operatorname{Hom}(P(\pi), \Delta(\pi))$. But $\operatorname{dim}_{q} \operatorname{Hom}(P(\pi), \Delta(\pi))=\operatorname{dim}_{q} \operatorname{Hom}(\Delta(\pi), \Delta(\pi))=\operatorname{dim}_{q} B_{\pi}$.

(iii) We need to prove that $\Delta(\pi) N_{\pi}$ is the smallest subobject $K$ of $\Delta(\pi)$ with $[\Delta(\pi) / K: L(\pi)]_{q}=1$. By part (ii), $\left[\Delta(\pi) / \Delta(\pi) N_{\pi}: L(\pi)\right]_{q}=1$. On the other hand, since $B_{\pi}$ is Noetherian, we can write $N_{\pi}=B_{\pi} x_{1}+\cdots+B_{\pi} x_{k}$ for some $x_{1}, \ldots, x_{n} \in B_{\pi}$ of positive degrees. So we have $\Delta(\pi) N_{\pi}=\Delta(\pi) x_{1}+\cdots+\Delta(\pi) x_{k}$. Moreover, each $\Delta(\pi) x_{m}$ has simple head $L(\pi)$. It follows that any non-trivial quotient of $\Delta(\pi) N_{\pi}$ has $L(\pi)$ as its quotient. This gives the required minimality property of $\Delta(\pi) N_{\pi}$.

(iv) By (iii), the definition of $\operatorname{rank}_{q}$ and (FGen), it suffices to prove that $\operatorname{Hom}\left(P(\sigma), \Delta(\pi) N_{\pi}\right)=\operatorname{Hom}(P(\sigma), \Delta(\pi)) N_{\pi}$. The containment ' $\supseteq$ ' is clear. On the other hand, let $f: q^{n} P(\sigma) \rightarrow \Delta(\pi) N_{\pi}$. Using the notation from the previous paragraph, we have a commutative diagram



with horizontal morphism being $\left(x_{1}, \ldots, x_{k}\right)$ and where $n_{k}=\operatorname{deg}\left(x_{k}\right)$. The projectivity of $P(\sigma)$ yields the morphism $f^{\prime}=\left(\begin{array}{c}f_{1} \\ \vdots \\ f_{k}\end{array}\right)$ which makes the diagram commutative, i.e. $f=x_{1} \circ f_{1}+\cdots+x_{k} \circ f_{k}=f_{1} x_{1}+\cdots+f_{k} x_{k} \in \operatorname{Hom}(P(\sigma), \Delta(\pi)) N_{\pi}$, as required.

(v) The filtration $\Delta(\pi) \supseteq \Delta(\pi) N_{\pi} \supseteq \Delta(\pi) N_{\pi}^{2} \supseteq \ldots$ is exhaustive by degrees. It remains to observe that for any $m$, the module $\Delta(\pi) N_{\pi}^{m} / \Delta(\pi) N_{\pi}^{m+1}$ has a finite filtration with subquotients isomorphic to quotients of modules of the form $q^{n} \bar{\Delta}(\pi)$ with $n>0$. Using the notation of the proof of (iii) above, we see that each $x_{i} \in B_{\pi}$ induces a morphism $\bar{x}_{i}: \Delta(\pi) N_{\pi}^{m-1} / \Delta(\pi) N_{\pi}^{m} \rightarrow \Delta(\pi) N_{\pi}^{m} / \Delta(\pi) N_{\pi}^{m+1}$, and $\Delta(\pi) N_{\pi}^{m} / \Delta(\pi) N_{\pi}^{m+1}$ is the sum of the images of $\bar{x}_{1}, \ldots, \bar{x}_{k}$. The result follows by induction on $m$.

Proposition 5.6 can be strengthened for $\mathscr{B}$-highest weight categories:

Proposition 5.7. Let $\mathbf{C}$ be a $\mathscr{B}$-highest weight category and $\pi \in \Pi$. Then:

(i) In the Grothendieck group $[\mathbf{C}]_{q}$, we have $[\Delta(\pi)]=\left(\operatorname{dim}_{q} B_{\pi}\right)[\bar{\Delta}(\pi)]$.

(ii) $\Delta(\pi)$ has an exhaustive filtration $\Delta(\pi) \supseteq \Delta(\pi) N_{\pi} \supseteq \Delta(\pi) N_{\pi}^{2} \supseteq \ldots$, and $\Delta(\pi) N_{\pi}^{n} / \Delta(\pi) N_{\pi}^{n+1} \cong\left(\operatorname{dim}_{q} N_{\pi}^{n} / N_{\pi}^{n+1}\right) \bar{\Delta}(\pi)$.

Proof. (i) Apply Proposition 5.6(iv) and the axiom (HWC).

(ii) We consider the exhaustive filtration $\Delta(\pi) \supseteq \Delta(\pi) N_{\pi} \supseteq \Delta(\pi) N_{\pi}^{2} \supseteq \ldots$. We can choose a homogeneous basis of $\left\{y_{a} \mid a \in A\right\}$ of $B_{\pi}$ and a partition $A=\sqcup_{n \geq 0} A_{n}$ in such a way that $\left\{y_{a}+N_{\pi}^{n+1} \mid a \in A_{n}\right\}$ is a basis of $N_{\pi}^{n} / N_{\pi}^{n+1}$ for all $n=0,1, \ldots$. Now, each $y_{a}$ with $a \in A_{n}$ induces a morphism $\bar{y}_{a}: \bar{\Delta}(\pi)=$ $\Delta(\pi) / \Delta(\pi) N_{\pi} \rightarrow \Delta(\pi) N_{\pi}^{n} / \Delta(\pi) N_{\pi}^{n+1}$. We have that

$$
\Delta(\pi) N_{\pi}^{n} / \Delta(\pi) N_{\pi}^{n+1}=\sum_{a \in A_{n}} \operatorname{im} \bar{y}_{a} .
$$


Moreover, each $\operatorname{im} \bar{y}_{a}$ is a quotient of $q^{\operatorname{deg}\left(y_{a}\right)} \bar{\Delta}(\pi)$. Since $\operatorname{dim}_{q} B_{\pi}=\sum_{a \in A} \operatorname{deg}\left(y_{a}\right)$, part (i) implies that each $\operatorname{im} \bar{y}_{a} \cong q^{\operatorname{deg}\left(y_{a}\right)} \bar{\Delta}(\pi)$ and the sum in (5.8) is direct.

Recall the notation of 4.1 . A version of the previous proposition for properly stratified categories is as follows:

Lemma 5.9. If $\mathbf{C}$ is $\mathscr{B}$-properly stratified, then for any $\pi \in \Pi$ the standard object $\Delta(\pi)$ has an exhaustive filtration whose factors are properly stratified modules of the form $q^{m} \bar{\Delta}(\sigma)$ for $m \in \mathbb{Z}$ with $\varrho(\sigma)=\varrho(\pi)$; moreover $q^{m} \bar{\Delta}(\sigma)$ appears the same (finite) amount of times as a composition factor $q^{m} L(\sigma)$ appears in $P_{\xi}(\tau)$.

Proof. Apply the exact functor $\mathcal{E}_{\xi}$ to a composition series of $P_{\xi}(\pi)$.

5.3. Extensions and $\Delta$-filtrations. For $\mathscr{B}$-stratified categories, Lemma 4.5)(i) can be strengthened as follows:

Lemma 5.10. Let $\mathbf{C}$ be a $\mathscr{B}$-stratified category and $X \in \mathbf{C}$.

(i) If $\operatorname{Ext}^{1}(\Delta(\pi), X) \neq 0$ then $[X: L(\sigma)]_{q} \neq 0$ for some $\sigma>\pi$. In particular, $\operatorname{Ext}^{1}(\Delta(\pi), \Delta(\sigma)) \neq 0$ implies $\pi<\sigma$.

(ii) Assume that every $K(\pi)$ has a finite $\Delta$-filtration, and $i \geq 1$. Then $\operatorname{Ext}^{i}(\Delta(\pi), X) \neq 0$ implies that $[X: L(\sigma)]_{q} \neq 0$ for some $\sigma>\pi$. In particular, $\operatorname{Ext}^{i}(\Delta(\pi), \Delta(\sigma)) \neq 0$ implies $\pi<\sigma$.

Proof. Using Lemma 3.8, we may assume that $X \simeq L(\sigma)$. The short exact sequence $0 \rightarrow K(\pi) \rightarrow P(\pi) \rightarrow \Delta(\pi) \rightarrow 0$ gives rise to the exact sequence

$$
0 \rightarrow \operatorname{Ext}^{i-1}(K(\pi), L(\sigma)) \rightarrow \operatorname{Ext}^{i}(\Delta(\pi), L(\sigma)) \rightarrow 0
$$

for all $i \geq 1$. So (i) will follow if we can show that $\operatorname{Hom}(K(\pi), L(\sigma))=0$ unless $\sigma>\pi$. Let $\sigma \ngtr \pi$. By (SC1), $K(\pi)$ has a $\Delta$-filtration $K(\pi)=K_{0} \supseteq K_{1} \supseteq \ldots$ with factors of the form $q^{m} \Delta(\tau)$ with $\tau>\sigma$. So $\operatorname{Hom}\left(K(\pi) / K_{n}, L(\sigma)\right)=0$ for all $n$. Assume for a contradiction that $\operatorname{hom}\left(K(\pi), q^{k} L(\sigma)\right) \neq 0$ for some $k$. As $\operatorname{hom}\left(K(\pi) / K_{n}, q^{k} L(\sigma)\right)=0$ for all $n$, it then follows that $\operatorname{hom}\left(K_{n}, q^{k} L(\sigma)\right) \neq 0$ for all $n$. In particular, $\operatorname{hom}\left(q^{k} P(\sigma), K_{n}\right) \neq 0$ for all $n$. Since the last hom-space is finite dimensional by Lemma 3.3(ii) and the filtration is exhaustive, we get a contradiction.

(ii) follows from (5.11) by induction on $i$.

Lemma 5.12. Let $\mathbf{C}$ be a $\mathscr{B}$-stratified category, $V=V_{0} \supseteq V_{1} \supseteq \ldots$ be a $\Delta$ filtration of an abject $V \in \mathbf{C}$, and $X(V):=\left\{\pi \in \Pi \mid V_{r} / V_{r+1} \simeq \Delta(\pi)\right.$ for some $\left.r\right\}$. Then:

(i) The set $X(V)_{\min }$ of minimal elements of $X(V)$ is finite.

(ii) $X(V) \subseteq \bigcup_{\pi \in X(V)_{\min }} \Pi_{\geq \pi}$; in particular, $X(V)_{\min }$ is non-empty.

(iii) For each $\pi \in \Pi$, there are only finitely many $r$ with $V_{r} / V_{r+1} \simeq \Delta(\pi)$.

Proof. Let $X^{\prime}=\left\{\pi_{1}, \ldots, \pi_{m}\right\}$ be the set of all $\pi$ such that there exists a $\Delta$ filtration of $V$ with the top subquotient $\simeq \Delta(\pi)$. The set $X^{\prime}$ is indeed finite by Lemma 3.3(iii).

(i) If $\pi \in X(V)_{\min }$, then by Lemma 5.10 (i), we have $\operatorname{Ext}^{1}(\Delta(\sigma), \Delta(\pi))=0$ for all $\sigma \in X(V)$. It follows that $\pi \in X^{\prime}$. In particular, $X(V)_{\min }$ is finite.

(ii) We first prove by induction on $n$ that the top $n$ subquotients of an arbitrary $\Delta$-filtration $V=W_{0} \supseteq W_{1} \supseteq \ldots$ are of the form $\simeq \Delta(\pi)$ with $\pi \in Y:=$ $\bigcup_{k=1}^{m} \Pi_{\geq \pi_{k}}$. The induction base $n=1$ is clear from the definitions. Let $n>1$ 
and $W_{n-1} / W_{n} \simeq \Delta(\pi)$. We have to prove that $\pi \in Y$. Let $W_{n-2} / W_{n-1} \simeq$ $\Delta(\sigma)$. By the inductive assumption, $\sigma \in Y$. If $\pi>\sigma$, then $\pi \in Y$. Otherwise, $\operatorname{Ext}^{1}(\Delta(\sigma), \Delta(\pi))=0$ by Lemma 5.10 (i). So we can reorder the levels of the $\Delta$-filtration so that $W_{n-2} / W_{n-1} \simeq \Delta(\pi)$, and $\pi \in Y$ again by the inductive assumption.

We deduce from the previous paragraph that $X(V) \subseteq Y$, which easily implies (ii) since $\Xi$ is interval-finite.

(iii) Let $\pi \in X(V)_{\min }$. If there are infinitely many $r$ with $V_{r} / V_{r+1} \simeq \Delta(\pi)$, then Lemma 5.10(i) allows us to reorder the filtration in such a way that arbitrarily large amount of its top subquotients is $\simeq \Delta(\pi)$, in which case, since $\operatorname{Ext}^{1}(\Delta(\pi), \Delta(\pi))=0$ again by Lemma $5.10(\mathrm{i})$, we have that $\operatorname{Hom}(V, L(\pi))$ is infinite dimensional, which contradicts Corollary 3.4(iii). Thus there are only finitely many $r$ with $V_{r} / V_{r+1} \simeq \Delta(\pi)$, and we can reorder the layers of the filtration to get a subobject $V^{\prime} \subseteq V$ having a $\Delta$-filtration such that $\Delta(\pi)$ does not arise as a subquotient the $\Delta$-filtration of $V^{\prime}$.

Using the assumption that $\Pi$ is interval finite, for any $\sigma \in X(V)$, after finitely many steps as above, we reach a subobject $V^{\prime \prime} \subset V$ with a $\Delta$-filtration $V^{\prime \prime}=$ $V_{0}^{\prime \prime} \supseteq V_{1}^{\prime \prime} \supseteq \ldots$ such that $\left|\left\{r \mid V_{r} / V_{r+1} \simeq \Delta(\sigma)\right\}\right|=\left|\left\{r \mid V_{r}^{\prime \prime} / V_{r+1}^{\prime \prime} \simeq \Delta(\sigma)\right\}\right|$ and $\sigma$ is minimal in $X\left(V^{\prime \prime}\right)$. The finiteness of $\left|\left\{r \mid V_{r}^{\prime \prime} / V_{r+1}^{\prime \prime} \simeq \Delta(\sigma)\right\}\right|$ now follows from the argument in the previous paragraph.

Corollary 5.13. Let $\mathbf{C}$ be a $\mathscr{B}$-stratified category, $V \in \mathbf{C}, V=V_{0} \supseteq V_{1} \supseteq \ldots$ be a $\Delta$-filtration, and $X(V):=\left\{\pi \in \Pi \mid V_{r} / V_{r+1} \simeq \Delta(\pi)\right.$ for some $\left.r\right\}$. Then:

(i) There is a $\Delta$-filtration $V=W_{0} \supseteq W_{1} \supseteq \ldots$ such that $W_{i} / W_{i+1} \simeq \Delta\left(\pi_{i}\right)$ for $i=0,1, \ldots$, and for all $i, j \geq 0$, we have that $\pi_{i}<\pi_{j}$ implies $i<j$.

(ii) If $\pi$ is a minimal element of $X(V)$, then there is a $\Delta$-filtration of $V$ with the top subquotient $\simeq \Delta(\pi)$.

(iii) If $X(V)$ is finite, and $\pi$ is a maximal element of $X(V)$, then there is a $\Delta$-filtration of $V$ with the bottom subquotient $\simeq \Delta(\pi)$.

Proof. Define the sets $X^{1}, X^{2}, \ldots$ recurrently from $X^{1}=X(V)_{\min }$ and $X^{n}=$ $\left(X \backslash\left(X^{1} \cup \cdots \cup X^{n-1}\right)\right)_{\min }$ for $n>0$. By Lemma 5.12 and since $\Pi$ is intervalfinite, we have $X=\sqcup_{n \geq 1} X^{n}$. We construct a filtration $V=V^{0} \supseteq V^{1} \supseteq V_{2} \supseteq \ldots$ recurrently as follows.

By Lemmas 5.12 and 5.10 (i), there is a subobject $V^{1} \subseteq V$ such that $V^{1}$ has a $\Delta$-filtration with subquotients of the form $\Delta(\sigma)$ with $\sigma \in X \backslash X^{1}$ and $V / V^{1}$ has a $\Delta$-filtration with subquotients of the form $\Delta(\sigma)$ with $\sigma \in X^{1}$. By the same argument, we get a subobject $V^{2} \subseteq V^{1}$ such that $V^{2}$ has a $\Delta$-filtration with subquotients of the form $\Delta(\sigma)$ with $\sigma \in X \backslash\left(X^{1} \cup X^{2}\right)$ and $V / V^{2}$ has a $\Delta$-filtration with subquotients of the form $\Delta(\sigma)$ with $\sigma \in X^{1} \cup X^{2}$. Continuing this way, we get an exhaustive $\Delta$-filtration $V=V^{0} \supseteq V^{1} \supseteq V^{2} \supseteq \ldots$ of $V$, where $V^{n-1} / V^{n}$ has $\Delta$-filtration with subquotients $\simeq \Delta(\pi)$ for $\pi \in X^{n}$. By Lemma 5.10 (i), $V^{n} / V^{n+1}$ is isomorphic to a direct sum of these subquotients. Parts (i) and (ii) now follow. Part (iii) is proved similarly, but starting from the maximal elements of $X(V)$.

5.4. Saturated sets. A subset $\Sigma \subseteq \Pi$ is called saturated if $\pi \in \Sigma$ whenever $\pi \leq \sigma$ and $\sigma \in \Sigma$.

Lemma 5.14. Let $\mathbf{C}$ be a $\mathscr{B}$-stratified category, $V=V_{0} \supseteq V_{1} \supseteq \ldots$ be a $\Delta$ filtration of an object $V \in \mathbf{C}$, and $X(V):=\left\{\pi \in \Pi \mid V_{r} / V_{r+1} \simeq \Delta(\pi)\right.$ for some $\left.r\right\}$. 
Let $\Sigma \subseteq \Pi$ be a saturated subset such that $\Sigma \cap X(V)$ is finite. Then there is a $\Delta$-filtration $V=W_{0} \supseteq W_{1} \supseteq \ldots$ such that the following two conditions hold:

(1) $\left|\left\{r \mid V_{r} / V_{r+1} \cong q^{n} \Delta(\pi)\right\}\right|=\left|\left\{r \mid W_{r} / W_{r+1} \cong q^{n} \Delta(\pi)\right\}\right|$ for every $n \in \mathbb{Z}$ and every $\pi \in \Pi$.

(2) there is $t \in \mathbb{Z}_{\geq 0}$ such that $W_{t}=\mathcal{O}^{\Sigma}(V), r<t$ implies $W_{r} / W_{r+1} \simeq \Delta(\sigma)$ for $\sigma \in \Sigma$, and $r \geq t$ implies $W_{r} / W_{r+1} \simeq \Delta(\pi)$ for $\pi \notin \Sigma$.

In particular, $\mathcal{O}^{\Sigma}(V)$ and $\mathcal{Q}^{\Sigma}(V)$ have $\Delta$-filtrations.

Proof. We use the notation of Lemma 5.12. We may assume that $X(V) \cap \Sigma \neq \emptyset$ since otherwise $\mathcal{O}^{\Sigma}(V)=V$. Let $X(V)_{\min }=X_{1} \sqcup X_{1}^{\prime}$, where $X_{1}=X(V)_{\min } \cap \Sigma$. By Lemma 5.12, $X_{1} \neq \emptyset$ since $\Sigma$ is saturated. By Corollary 5.13(ii), there exists a subobject $V^{1} \subseteq V$ such that $V / V^{1}$ has a finite $\Delta$-filtration with subquotients $\simeq \Delta(\pi)$ for $\pi \in X_{1}$ and $V_{1}$ has a $\Delta$-filtration with subquotients $\simeq \Delta(\pi)$ for $\pi \in X(V) \backslash X_{1}$. Note that $O^{\Sigma}(V) \subseteq V_{1}$, hence $\mathcal{O}^{\Sigma}(V)=\mathcal{O}^{\Sigma}\left(V_{1}\right)$. Repeating this procedure finitely many times, we get a chain of submodules $V \supset V^{1} \supset V^{2} \supset$ $\cdots \supseteq V^{l}=\mathcal{O}^{\Sigma}(V)$, and the result follows.

Let $V=V_{0} \supseteq V_{1} \supseteq \ldots$ be a $\Delta$-filtration. Define the corresponding $\Delta$ multiplicities as follows

$$
(V: \Delta(\pi))_{q}:=\sum_{n \in \mathbb{Z}}\left|\left\{r \mid V_{r} / V_{r+1} \cong q^{n} \Delta(\pi)\right\}\right| q^{n} \quad(\pi \in \Pi) .
$$

In view of Lemma 5.12(iii), we have $(V: \Delta(\pi))_{q} \in \mathbb{Z}\left[q, q^{-1}\right]$.

Lemma 5.15. Let $\mathbf{C}$ be a $\mathscr{B}$-stratified category and $V$ be an object with a $\Delta$ filtration. Then for each $\pi \in \Pi$, the multiplicity $(V: \Delta(\pi))_{q}$ does not depend on the choice of a $\Delta$-filtration of $V$.

Proof. Let $X(V):=\left\{\pi \in \Pi \mid V_{r} / V_{r+1} \simeq \Delta(\pi)\right.$ for some $\left.r\right\}$. By Lemma 5.12, $X(V) \subseteq \bigcup_{\pi \in X(V)_{\min }} \Pi_{\geq \pi}$. Fix an arbitrary $\sigma \in \Pi$ and consider the saturated set $\Sigma:=\Pi_{\leq \sigma}$. Since $\Pi$ is interval finite, $X(V) \cap \Sigma$ is finite. By Lemma 5.14, $Q^{\Sigma}(V)$ has a finite $\Delta$-filtration with $\left(Q^{\Sigma}(V): \Delta(\tau)\right)_{q}=(V: \Delta(\tau))_{q}$ for all $\tau \in \Sigma \cap X(V)$. In this way, we see that to check that $(V: \Delta(\pi))_{q}$ does not depend on the choice of a $\Delta$-filtration of $V$ can be reduced to the case where $V$ has a finite $\Delta$-filtration. The finite filtration case follows by a Grothendieck group argument.

Proposition 5.16. Let $\Sigma \subseteq \Pi$ be a finite saturated subset. If the category $\mathbf{C}$ is $\mathscr{B}$-stratified, (resp. standardly $\mathscr{B}$-stratified, properly $\mathscr{B}$-stratified, $\mathscr{B}$-highest weight, weakly $\mathscr{B}$-highest weight) with respect to $\varrho: \Pi \rightarrow \Xi$, then so is $\mathbf{C}(\Sigma)$ with respect to $\left.\varrho\right|_{\Sigma}: \Sigma \rightarrow \varrho(\Sigma)$; the standard objects for $\mathbf{C}(\Sigma)$ are $\{\Delta(\sigma) \mid \sigma \in \Sigma\}$.

Proof. By Lemma 3.10, the category $\mathbf{C}(\Sigma)$ is graded Noetherian Laurentian. Let $\sigma \in \Sigma$. Then the standard object $\Delta(\sigma) \in \mathbf{C}$ belongs to $\mathbf{C}(\Sigma)$. Moreover, by Lemma [5.14, the projective cover $\mathcal{Q}^{\Sigma}(P(\sigma))$ of $L(\sigma)$ in $\mathbf{C}(\Sigma)$ has a $\Delta$-filtration with factors $\simeq \Delta(\pi)$ for $\pi \in \Sigma$ such that $\pi>\sigma$. Now, it is easy to see that $\Delta(\sigma)$ is indeed a standard object in $\mathbf{C}(\Sigma)$, as defined in (4.2). Finally, the properties (SC2), (FGen), (PSC), (SSC), (HWC), (FGen) for $\mathbf{C}(\Sigma)$ get inherited from $\mathbf{C}$.

5.5. Homological dimensions. For a subset $\Sigma \subseteq \Pi$ recall $l(\Sigma) \in \mathbb{Z}_{\geq 0} \cup\{\infty\}$ defined in (1.1).

Lemma 5.17. If $\mathbf{C}$ is a $\mathscr{B}$-stratified category and $\pi \in \Pi$, then proj.dim $\Delta(\pi) \leq$ $l\left(\Pi_{\geq \pi}\right)$. 
Proof. We may assume that $l\left(\Pi_{\geq \pi}\right)$ is finite. Apply induction on $l\left(\Pi_{\geq \pi}\right)$. If $l\left(\Pi_{\geq \pi}\right)=0$ then $\pi$ is a maximal element in $\Pi$, and $\Delta(\pi)$ is projective by (SC1), giving the induction base. If $l\left(\Pi_{\geq \pi}\right)>0$, then by (SC1) and Lemma 5.12 (iii), $K(\pi)$ has a finite filtration with factors $\simeq \Delta(\sigma)$ with $\sigma>\pi$. For such $\sigma$, we have $l\left(\Pi_{\geq \sigma}\right)<l\left(\Pi_{\geq \pi}\right)$, and by the inductive assumption proj. $\operatorname{dim} \Delta(\sigma)<l\left(\Pi_{\geq \pi}\right)$. So proj. $\operatorname{dim} K(\pi)<l\left(\Pi_{\geq \pi}\right)$ and proj. $\operatorname{dim} \Delta(\pi) \leq$ proj. $\operatorname{dim} K(\pi)+1 \leq l\left(\Pi_{\geq \pi}\right)$.

Let $\xi \in \Xi$. Denote the (possibly infinite) global dimension of $B_{\xi}$ (as a graded algebra) by

$$
d_{\xi}:=\text { gl. } \operatorname{dim} B_{\xi} \quad\left(\xi \in B_{\xi}\right) .
$$

Lemma 5.18. Let $\mathbf{C}$ be a properly $\mathscr{B}$-stratified category, and $\pi \in \Pi$ with $\varrho(\pi)=$ $\xi$. Then there exists an exact sequence

$$
\cdots \rightarrow Z_{2} \stackrel{\partial_{2}}{\longrightarrow} Z_{1} \stackrel{\partial_{1}}{\longrightarrow} Z_{0} \stackrel{\partial_{0}}{\longrightarrow} \bar{\Delta}(\pi) \rightarrow 0,
$$

with each $Z_{k}$ being a finite direct sum of modules of the form $q^{m} \Delta(\sigma)$ with $m \in \mathbb{Z}$ and $\varrho(\sigma)=\xi$, such that $Z_{n}=0$ for all $n>d_{\xi}$.

Proof. Apply the standardizetion functor $\mathcal{E}_{\xi}$ to a minimal projective resolution of $L(\pi)$ in $\mathbf{C}_{\xi}$.

A version of the previous lemma for $\mathscr{B}$-highest weight categories (when $\Pi=\Xi$ ) is as follows

Lemma 5.20. Let $\mathbf{C}$ be a $\mathscr{B}$-highest weight category, and $\pi \in \Pi$. Then there exists an exact sequence

$$
\cdots \rightarrow Z_{2} \stackrel{\partial_{2}}{\longrightarrow} Z_{1} \stackrel{\partial_{1}}{\longrightarrow} Z_{0} \stackrel{\partial_{0}}{\longrightarrow} \bar{\Delta}(\pi) \rightarrow 0,
$$

with each $Z_{k}$ being a finite direct sum of modules of the form $q^{m} \Delta(\pi)$, such that $Z_{n}=0$ for all $n>d_{\pi}$.

Proof. We have a free resolution $\mathbf{F}$ of the right $B_{\pi}$-module $B_{\pi} / N_{\pi}$

$$
0 \rightarrow F_{d_{\pi}} \stackrel{\partial_{d_{\pi}}^{\prime}}{\longrightarrow} \cdots \stackrel{\partial_{2}^{\prime}}{\longrightarrow} F_{1} \stackrel{\partial_{1}^{\prime}}{\longrightarrow} F_{0} \stackrel{\partial_{0}^{\prime}}{\longrightarrow} B_{\pi} / N_{\pi} \rightarrow 0
$$

with the free modules $F_{i}$ of the form

$$
F_{i}=q^{m_{i 1}} B_{\pi} \oplus \cdots \oplus q^{m_{i l_{i}}} B_{\pi} \quad\left(i=0,1, \ldots, d_{\pi}\right),
$$

and the boundary maps $\partial_{i}$ given by the left multiplication with the matrices $M^{i}=\left(b_{r s}^{i}\right)_{1 \leq r \leq l_{i-1}, 1 \leq s \leq l_{i}}$ with entries in $B_{\pi}$. Now define

$$
Z_{i}:=q^{m_{i 1}} \Delta(\pi) \oplus \cdots \oplus q^{m_{i l_{i}}} \Delta(\pi) \quad(i=1,2, \ldots),
$$

and let the map $\partial_{i}: Z_{i} \rightarrow Z_{i-1}$ be given by multiplication with the matrix $M^{i}$, which makes sense since each $b_{r s}^{i} \in B_{\pi}=\operatorname{Hom}(\Delta(\pi), \Delta(\pi))$. It is clear that this gives a complex $\mathbf{Z}$ of the form (5.21). To prove that it is exact, we use the criterion of Lemma 3.7. For $\sigma \in \Pi$, the module $X:=\operatorname{Hom}(P(\sigma), \Delta(\pi))_{B_{\pi}}$ is free finite rank by (HWC), and the application of the functor $\operatorname{Hom}(P(\sigma),-)$ to the complex $\mathbf{Z}$ gives a compex isomorphic to $X \otimes_{B_{\pi}} \mathbf{F}$, which is exact.

Lemma 5.22. Let $\mathbf{C}$ be a properly $\mathscr{B}$-stratified or a $\mathscr{B}$-highest weight category, $\pi \in \Pi$, and $\xi=\varrho(\pi)$. Then

$$
\text { proj. } \operatorname{dim} \bar{\Delta}(\pi) \leq l\left(\Pi_{\geq \pi}\right)+d_{\xi} .
$$

Proof. This is a standard consequence of Lemmas 5.18, 5.20 and 5.17, 
Define $d_{\rho}:=\max \left\{d_{\xi} \mid \xi \in \Xi\right\}$.

Proposition 5.23. Let $\mathbf{C}$ be a properly $\mathscr{B}$-stratified or a $\mathscr{B}$-highest weight category, and $\pi, \sigma \in \Pi$. Then $\operatorname{Ext}^{i}(L(\pi), L(\sigma))=0$ for all $i>l\left(\Pi_{\leq \pi}\right)+l(\Pi)+d_{\rho}$. In particular, if $i>2 l(\Pi)+d_{\rho}$, then $\operatorname{Ext}^{i}\left(L, L^{\prime}\right)=0$ for all simple objects $L, L^{\prime}$.

Proof. We may assume that $d_{\rho}$ and $l(\Pi)$ are finite. Then $l\left(\Pi_{\leq \pi}\right)$ is also finite. We apply induction on $l\left(\Pi_{\leq \pi}\right)$. If $l\left(\Pi_{\leq \pi}\right)=0$, then $L(\pi)=\bar{\Delta}(\pi)$, and the result follows from Lemma $\overline{5.22}$. Let $l\left(\Pi_{\leq \pi}\right)>0$. The short exact sequence $0 \rightarrow K \rightarrow \bar{\Delta}(\pi) \rightarrow L(\pi) \rightarrow 0$ yields the exact sequence in cohomology:

$$
\operatorname{Ext}^{i-1}(K, L(\sigma)) \rightarrow \operatorname{Ext}^{i}(L(\pi), L(\sigma)) \rightarrow \operatorname{Ext}^{i}(\bar{\Delta}(\pi), L(\sigma)),
$$

with the last term being zero by Lemma 5.22 again. So it remains to prove that the first term is zero.

All composition factors of $K$ are $\simeq L(\kappa)$ with $\kappa<\pi$. Note $l\left(\Pi_{\leq \pi}\right)-1 \geq l\left(\Pi_{\leq \kappa}\right)$ for all such $\kappa$. Let $i>l\left(\Pi_{\leq \pi}\right)+l(\Pi)+d_{\rho}$. Then $i-1>l\left(\Pi_{\leq \kappa}\right)+l(\Pi)+d_{\rho}$ for all $\kappa<\pi$, and so $\operatorname{Ext}^{i-1}(K, \bar{L}(\sigma))=0$ by the inductive assumption using the fact that $K$ has finite length in view of Proposition 5.6(iv).

Lemma 5.24. If $l(\Pi)<\infty$, then for every object in a $\mathscr{B}$-stratified category $\mathbf{C}$ there are only finitely many $\pi \in \Pi$ with $\operatorname{Hom}(P(\pi), C) \neq 0$.

Proof. By Lemma 3.3(i), we may assume that $C \cong P(\sigma)$. But $P(\sigma)$ has a filtration with factors $\simeq \Delta(\kappa)$ for $\kappa \geq \sigma$, and there are only finitely many such $\kappa$ 's by assumption. Furthermore, $\operatorname{Hom}(P(\pi), \Delta(\kappa)) \neq 0$ implies $\pi \leq \kappa$, and there are only finitely many such $\pi$ 's by assumption.

Recall the notion of the global dimension of an abelian category [41, §VII.6].

Corollary 5.25. If $\mathbf{C}$ is a $\mathscr{B}$-properly stratified or a $\mathscr{B}$-highest weight category, then the global dimension of $\mathbf{C}$ is at most $2 l(\Pi)+d_{\rho}$.

Proof. We follow the ideas of [40, Lemma 4.10 and proof of Theorem 4.6]. Let $i>2 l(\Pi)+d_{\rho}$. By Lemma 3.8, it suffices to prove that $\operatorname{Ext}^{i}(C, L)=0$ for an arbitrary $C \in \mathbf{C}$ and simple $L \in \mathbf{C}$. For this to suffices to prove that $\operatorname{ext}^{i}(C, L)=$ 0 . If $C$ is finite length, the result follows from Proposition 5.23.

In general, let $C \supseteq C_{1} \supseteq C_{2} \supseteq \ldots$ be a filtration as in (NLC2). Since $C / C_{n}$ is finite length, it suffices to prove that $\operatorname{ext}^{i}\left(C_{n}, L\right)=0$ for $n$ sufficiently large. By Corollary 3.4(i), $C_{n}$ has a projective resolution $\cdots \rightarrow P_{1} \rightarrow P_{0}$, with $P_{i}$ being a finite direct sum of modules of the form $q^{m} P(\sigma)$. Moreover, it follows from (NLC3) and Lemmas 3.3(iii), 5.24 that for $n \gg 0$ we also have $m \gg 0$ for all summands $q^{m} P(\sigma)$ of $P_{i}$. So hom $\left(P_{i}, L\right)=0$ and $\operatorname{ext}^{i}\left(C_{n}, L\right)=0$ for $n \gg 0$.

Finally, we strengthen Lemma 4.5(ii):

Lemma 5.26. Let $\mathbf{C}$ be a $\mathscr{B}$-highest weight category and $X \in \mathbf{C}$.

(i) $\operatorname{Ext}^{1}(\bar{\Delta}(\pi), X) \neq 0$ implies that $X$ has a subquotient $\simeq L(\sigma)$ for $\sigma \geq \pi$. In particular, $\operatorname{Ext}^{1}(\bar{\Delta}(\pi), \bar{\Delta}(\sigma)) \neq 0$ implies $\pi \leq \sigma$.

(ii) Assume that every $K(\pi)$ has a finite $\Delta$-filtration, and $i \geq 1$. Then $\operatorname{Ext}^{i}(\bar{\Delta}(\pi), X) \neq 0$ implies that $X$ has a subquotient $\simeq L(\sigma)$ for $\sigma \geq \pi$. In particular, $\operatorname{Ext}^{i}(\bar{\Delta}(\pi), \bar{\Delta}(\sigma)) \neq 0$ implies $\pi \leq \sigma$. 
Proof. (i) Using Lemma 3.8, we may assume that $X \simeq L(\sigma)$. If $\sigma \nsupseteq \pi$, then $\sigma \ngtr \pi$, so $\operatorname{Ext}^{1}(\Delta(\pi), L(\sigma))=0$ by Lemma 5.10)(i). Now use the resolution (5.19) or (5.21) of $\bar{\Delta}(\pi)$ to see that $\operatorname{Ext}^{1}(\bar{\Delta}(\pi), L(\sigma))$. The proof of (ii) is similar, but uses Lemma 5.10 (ii).

\section{6. $\mathscr{B}$-QUASIHEREDITARY AND $\mathscr{B}$-STRATIFIED ALGEBRAS}

Let $\mathbf{C}$ be a graded Noetherian Laurentian category with $|\Pi|<\infty$, i.e. with finitely many simple objects up to isomorphism and degree shift. By Theorem 3.9. the category $\mathbf{C}$ is equivalent to the category $H$-mod of finitely generated graded modules over a left Noetherian Laurentian graded algebra $H$. Let $\mathscr{B}$ be a fixed class of left Noetherian Laurentian algebras. In this section we introduce and study several versions of $\mathscr{B}$-stratified algebras. The main result is that the algebra $H$ is $\mathscr{B}$-stratified (resp. $\mathscr{B}$-properly stratified, resp. $\mathscr{B}$-standardly stratified, resp. $\mathscr{B}$-quasihereditary, weakly $\mathscr{B}$-quasihereditary) if and only if the category $H$-mod is $\mathscr{B}$-stratified (resp. $\mathscr{B}$-properly stratified, resp. $\mathscr{B}$-standardly stratified, resp. $\mathscr{B}$-highest weight, resp. weakly $\mathscr{B}$-highest weight), cf. [12], [14].

6.1. $\mathscr{B}$-stratified algebras. Let $H$ be a left Noetherian Laurentian algebra. We adopt the notation of $₫ 2.2$. In particular, for every $\pi \in \Pi$ we have a projective indecomposable module $P(\pi)$.

Definition 6.1. A two-sided ideal $J \subseteq H$ is called $\mathscr{B}$-stratifying if it satisfies the following conditions:

(SI1) $\operatorname{Hom}_{H}(J, H / J)=0$;

(SI2) As a left module, $J \cong \bigoplus_{\pi \in \xi} m_{\pi}(q) P(\pi)$ for some graded multiplicities $m_{\pi}(q) \in \mathbb{Z}\left[q, q^{-1}\right]$ and some subset $\xi \subseteq \Pi$, such that, setting $P_{\xi}:=$ $\bigoplus_{\pi \in \xi} P(\pi)$, we have $B_{\xi}:=\operatorname{End}_{H}\left(P_{\xi}\right)^{\text {op }} \in \mathscr{B}$.

A $\mathscr{B}$-stratifying ideal is called $\mathscr{B}$-standardly stratifying, if

(SSI) As a right $B_{\xi}$-module, $P_{\xi}$ is finitely generated.

A $\mathscr{B}$-stratifying ideal is called $\mathscr{B}$-properly stratifying, if

(PSI) As a right $B_{\xi}$-module, $P_{\xi}$ is finitely generated and flat.

Definition 6.2. Let $\mathscr{B}$ be a class of connected algebras. An ideal $J \subseteq H$ is called a $\mathscr{B}$-heredity ideal if it is $\mathscr{B}$-properly stratifying as in the definition above with $|\xi|=1$. An ideal $J \subseteq H$ is called a weakly $\mathscr{B}$-heredity ideal if it is $\mathscr{B}$-standardly stratifying as in the definition above with $|\xi|=1$.

Remark 6.3. Using the fact that for a connected algebra, a finitely generated module is flat if and only if it is free, we can restate the definition of a $\mathscr{B}$-heredity ideal (resp. weakly $\mathscr{B}$-heredity ideal) as follows: it is an ideal $J$ which satisfies (SI1) and such that as a left module, $J \cong m(q) P(\pi)$ for some graded multiplicity $m(q) \in \mathbb{Z}\left[q, q^{-1}\right]$ and some $\pi \in \Pi$, such that $B_{\pi}:=\operatorname{End}\left(P(\pi)^{\text {op }} \in \mathscr{B}\right.$ and $P(\pi)$ is free finite rank (resp. finitely generated) as a right $B_{\pi}$-module.

Definition 6.4. The algebra $H$ is called $\mathscr{B}$-stratified (resp. $\mathscr{B}$-standardly stratified, resp. $\mathscr{B}$-properly stratified, resp. (weakly) $\mathscr{B}$-quasihereditary) if there exists a finite chain of ideals

$$
H=J_{0} \supsetneq J_{1} \supsetneq \cdots \supsetneq J_{n}=(0)
$$


with $J_{i} / J_{i+1}$ a $\mathscr{B}$-stratifying (resp. $\mathscr{B}$-standardly stratifying, resp. $\mathscr{B}$-properly stratifying, resp. (weakly) $\mathscr{B}$-heredity) ideal in $H / J_{i+1}$ for all $0 \leq i<n$. Such a chain of ideals is called a $\mathscr{B}$-stratifying (resp. $\mathscr{B}$-standardly stratifying, resp. $\mathscr{B}$-properly stratifying, resp. (weakly) $\mathscr{B}$-heredity) chain.

The following two lemmas are basically known.

Lemma 6.5. Let $J$ be an ideal in $H$ such that the left $H$-module ${ }_{H} J$ is projective. Then the condition (SI1) is equivalent to the condition $J^{2}=J$, which in turn is equivalent to $\mathrm{J}=\mathrm{HeH}$ for an idempotent $e \in H$.

Proof. Use Lemma 2.8 and [17, Statement 2].

Lemma 6.6. Let $J$ be a weakly $\mathscr{B}$-heredity ideal in $H$. Write $J=H e H$ for an idempotent e, according to Lemma 6.5. Then the natural map $\mathrm{He} \otimes_{e \mathrm{He}} \mathrm{eH} \rightarrow J$ is an isomorphism. Moreover, we may choose an idempotent e to be primitive so that, using the notation of Remark 6.3, we have $\mathrm{He} \cong P(\pi)$ and $B_{\pi} \cong e H e$.

Proof. For the first statement see [17, Statement 7]. For the second statement, by Remark 6.3, we know that ${ }_{H} H e H \cong m(q) P(\pi)$. Let $e^{\prime}$ be a primitive idempotent such that $H e^{\prime} \simeq P(\pi)$. Then $H e^{\prime} H=\sum_{f \in \operatorname{Hom}_{H}(P(\pi), H)}$ im $f$. But by (SI1), we have $\sum_{f \in \operatorname{Hom}_{H}(P(\pi), H)} \operatorname{im} f=J$.

6.2. $\mathscr{B}$-analogue of Cline-Parshall-Scott Theorem. The following is a version of the fundamental theorem of Cline-Parshall-Scott [12], [14, (2.2.3)].

Theorem 6.7. Let $H$ be a left Noetherian Laurentian algebra. Then the category $H$-mod of finitely generated graded $H$-modules is a is $\mathscr{B}$-stratified (resp. $\mathscr{B}$ properly stratified, resp. $\mathscr{B}$-standardly stratified, resp. weakly $\mathscr{B}$-highest weight, resp. $\mathscr{B}$-highest weight) category if and only if $H$ is a $\mathscr{B}$-stratified (resp. $\mathscr{B}$ properly stratified, resp. $\mathscr{B}$-standardly stratified, resp. weakly $\mathscr{B}$-quasihereditary, resp. $\mathscr{B}$-quasihereditary) algebra. Moreover:

(i) Suppose that $H$-mod is $\mathscr{B}$-stratified with respect to $\varrho: \Pi \rightarrow \Xi$. Let $\Xi=\left\{\xi_{1}, \ldots, \xi_{n}\right\}$ with $\xi_{i}<\xi_{j}$ only if $i<j$, and define $\Sigma(i):=\varrho^{-1}\left(\xi_{1}\right) \cup$ $\cdots \cup \varrho^{-1}\left(\pi_{i}\right)$ for $1 \leq i \leq n$. Then

$$
H \supsetneq \mathcal{O}^{\Sigma(1)}(H) \supsetneq \mathcal{O}^{\Sigma(2)}(H) \supsetneq \cdots \supsetneq \mathcal{O}^{\Sigma(n)}(H)=0
$$

is a $\mathscr{B}$-stratifying chain of ideals. Moreover, if $H$-mod is $\mathscr{B}$-properly stratified (resp. $\mathscr{B}$-standardly stratified, resp. weakly $\mathscr{B}$-highest weight, resp. $\mathscr{B}$-highest weight), then the chain is $\mathscr{B}$-properly stratifying (resp. $\mathscr{B}$-standardly stratifying, resp. weakly $\mathscr{B}$-heredity, resp. $\mathscr{B}$-heredity).

(ii) Suppose that $H=J_{0} \supsetneq J_{1} \supsetneq \cdots \supsetneq J_{n}=(0)$ is a $\mathscr{B}$-stratifying (resp. $\mathscr{B}$-properly stratifying, resp. $\mathscr{B}$-standardly stratifying, resp. weakly $\mathscr{B}$ heredity, resp. $\mathscr{B}$-heredity) chain. Let $\Xi:=\{1,2, \ldots, n\}$ (standardly ordered), and for $\pi \in \Pi$ define

$$
\varrho(\pi):=\min \left\{i \mid\left[H / J_{i}: L(\pi)\right] \neq 0\right\} .
$$

Then the category $H$-mod is $\mathscr{B}$-stratified (resp. $\mathscr{B}$-properly stratified, resp. $\mathscr{B}$-standardly stratified, resp. weakly $\mathscr{B}$-highest weight, resp. $\mathscr{B}$ highest weight) with respect to $\varrho$. 
Proof. (i) We first show that if $\xi$ is a maximal element of $\Xi$, and $\Sigma:=\Pi \backslash\left\{\rho^{-1}(\xi)\right\}$, then $J:=\mathcal{O}^{\Sigma}(H)$ is a $\mathscr{B}$-stratifying (resp. $\mathscr{B}$-standardly stratifying, resp. $\mathscr{B}$ properly stratifying) ideal in $H$. By (2.5), (SC1), and Lemma 5.12(iii), the regular module ${ }_{H} H$ has a finite $\Delta$-filtration. So by Lemma [5.14, the module ${ }_{H} J$ has a finite filtration with sections $\simeq \Delta(\pi)$ for $\pi \in \rho^{-1}(\xi)$. Hence by Lemma 5.10 (i), we have that ${ }_{H} J$ is a finite direct sum of modules $\simeq \Delta(\pi)$ with $\pi \in \rho^{-1}(\xi)$. On the other hand, by the maximality of $\xi$ and (SC1), we have $P(\pi) \cong \Delta(\pi)$ for all $\pi \in \rho^{-1}(\xi)$, hence we have (SI2). Moreover

$$
\operatorname{dim}_{q} \operatorname{Hom}_{H}(\Delta(\pi), H / J)=\operatorname{dim}_{q} \operatorname{Hom}_{H}(P(\pi), H / J)=[H / J: L(\pi)]_{q}=0
$$

for all $\pi \in \rho^{-1}(\xi)$, hence $\operatorname{Hom}_{H}(J, H / J)=0$, which is (SI1). In view of Remark [5.3(ii), the property (FGen) implies the property (SSI) for $J$, and the property (PSC) implies the property (PSI) for $J$.

Finally, we need to establish that $\mathcal{O}^{\Sigma(i)}(H) / \mathcal{O}^{\Sigma(i+1)}(H)$ is a $\mathscr{B}$-stratifying (resp. $\mathscr{B}$-standardly stratifying, resp. $\mathscr{B}$-properly stratifying) ideal in the algebra $H / \mathcal{O}^{\Sigma(i+1)}(H)=H(\Sigma(i+1))$ for $0 \leq i<n$. Note that

$$
\mathcal{O}^{\Sigma(i)}(H) / \mathcal{O}^{\Sigma(i+1)}(H)=\mathcal{O}^{\Sigma(i)}(H(\Sigma(i+1))),
$$

and the required fact follows by Proposition 5.16 and the previous paragraph.

(ii) We apply induction on $n$ which starts with the trivial case $n=0$ (or with the easy case $n=1)$. Let $n \geq 1$. Set $J:=J_{n-1}$ and $\Sigma:=\{\pi \in \Pi \mid[H / J$ : $\left.L(\pi)]_{q} \neq 0\right\}$. By the inductive assumption, $H / J$-mod is a $\mathscr{B}$-stratified (resp. $\mathscr{B}-$ properly stratified, resp. $\mathscr{B}$-standardly stratified, resp. weakly $\mathscr{B}$-highest weight, resp. $\mathscr{B}$-highest weight) with respect to $\left.\varrho\right|_{\Sigma}: \Sigma \rightarrow\{1, \ldots, n-1\}$.

By (SI2), we have $J \cong \bigoplus_{\pi \in \xi} m_{\pi}(q) P(\pi)$ for some non-zero multiplicities $m_{\pi}(q) \in \mathbb{Z}\left[q, q^{-1}\right]$ and some $\xi \subseteq \Pi$. If there is $\pi \in \Sigma \cap \xi$, then $\operatorname{Hom}_{H}(J, H / J) \neq 0$, which contradicts (SI1). We deduce that $\xi=\Pi \backslash \Sigma$, that $[H / J: L(\pi)]_{q}=0$ for all $\pi \in \xi$, and that $J=\mathcal{O}^{\Sigma}(H)$. In particular, $\varrho(\pi)=n$ for all $\pi \in \xi$, and so all such $\pi$ are maximal. It follows that $P(\pi)=\Delta(\pi)$ for all $\pi \in \xi$ and that $\Delta_{n}$ satisfies (SC2), thanks to (SI2). Moreover, in view of Remark 5.3(ii), (SSI) (resp. (PSI)) implies that $\Delta_{n}$ satisfies (FGen) (resp. (PSC)).

Fix an arbitrary $\sigma \in \Sigma$. Denote $R(\sigma):=\mathcal{O}^{\Sigma}(P(\sigma))=J P(\sigma)$, see Lemma 3.12. By Lemma 3.10, $P_{0}(\sigma):=P(\sigma) / R(\sigma)$ is a projective cover of $L(\sigma)$ as modules over $H(\Sigma)=H / J$, and by definition $\Delta_{0}(\sigma)=\mathcal{Q}^{\leq \sigma}\left(P_{0}(\sigma)\right)$ is the corresponding standard module. Working in the (weakly) $\mathscr{B}$-highest weight category $H(\Sigma)$-mod, let $K_{0}(\sigma)=\mathcal{O}^{\leq \sigma}\left(P_{0}(\Sigma)\right)$. As $J$ is a projective $H$-module and $P(\sigma)$ is a direct summand of $H$, we get that $R(\sigma)=J P(\sigma)$ is also a projective $H$-module. Moreover, $R(\sigma)$ has no quotient belonging to $\Sigma$. Hence $R(\sigma)$ is a direct sum of modules $\simeq P(\pi) \cong \Delta(\pi)$ with $\pi \in \xi$. In particular, $\mathcal{Q}^{\leq \sigma}(R(\sigma))=0$. So the short exact sequence

$$
0 \rightarrow R(\sigma) \rightarrow P(\sigma) \rightarrow P_{0}(\sigma) \rightarrow 0
$$

induces an isomorphism $\Delta(\sigma) \rightarrow \Delta_{0}(\sigma)$. In particular, by the inductive assumption we have that $\Delta(\sigma)$ satisfies the properties (SC2), (FGen), and (PSC) (resp. (HWC)).

Moreover, there is a short exact sequence $0 \rightarrow R(\sigma) \rightarrow K(\sigma) \rightarrow K_{0}(\sigma) \rightarrow 0$. By the inductive assumption, $K_{0}(\sigma)$ has a filtration with sections $\simeq \Delta(\kappa)$ for $\sigma<\kappa \in \Sigma$, and we have shown that $R(\sigma)$ has filtration with sections $\simeq \Delta(\pi)$ for $\pi \in \xi$. Since $\pi>\sigma$ for all $\pi \in \xi$, we get the property (SC1) for $H$-mod. 
Corollary 6.8. Let $\mathbf{C}$ be a $\mathscr{B}$-stratified (resp. $\mathscr{B}$-properly stratified, resp. $\mathscr{B}$ standardly stratified, resp. weakly $\mathscr{B}$-highest weight, resp. $\mathscr{B}$-highest weight) with respect to $\varrho: \Pi \rightarrow \Xi$, and assume that $\Pi$ is finite. Then $\mathbf{C}$ is graded equivalent to $H$-mod for some $\mathscr{B}$-stratified (resp. $\mathscr{B}$-properly stratified, resp. $\mathscr{B}$-standardly stratified, resp. weakly $\mathscr{B}$-quasihereditary, resp. $\mathscr{B}$-quasihereditary) algebra $H$.

\section{Proper costandard modules}

In 97.1 we use $\mathscr{B}$-stratified algebras to construct proper costandard objects in $\mathscr{B}$-stratified categories with finite $\Pi$. In 97.2 , we apply proper costandard modules to deduce usual nice properties of good filtrations. In $\S \$ 7.317 .4$ we deal with more general $\mathscr{B}$-stratified categories.

7.1. Proper costandard modules. Throughout the subsection, $H$ is a $\mathscr{B}$ stratified algebra with a complete set of irreducible modules $\{L(\pi) \mid \pi \in \Pi\}$ up to isomorphism and degree shift. By Theorem 6.7, the category $H$-mod of finitely generated graded $H$-modules is $\mathscr{B}$-stratified with respect to some $\varrho: \Pi \rightarrow \Xi$.

For every $\pi \in \Pi$, let $I(\pi)$ be the injective envelope of $L(\lambda)$ in the category of all graded $H$-modules. In general (and in most interesting cases), the module $I(\pi)$ is not finitely generated and not Laurentian. Consider the short exact sequence

$$
0 \longrightarrow L(\pi) \longrightarrow I(\pi) \stackrel{\mathrm{p}}{\longrightarrow} I(\pi) / L(\pi) \longrightarrow 0 .
$$

As usual, $\varrho$ defines a partial preorder $\leq$ on $\Pi$. Let $A(\pi)$ be the largest submodule of $I(\pi) / L(\pi)$ all of whose irreducible subquotients are of the form $\simeq L(\sigma)$ for $\sigma<\pi$. Define the corresponding proper costandard module:

$$
\bar{\nabla}(\pi)=\mathrm{p}^{-1}(A(\pi)) .
$$

Lemma 7.2. For any $\pi, \sigma \in \Pi$, we have $\operatorname{dim}_{q} \operatorname{Hom}_{H}(\Delta(\sigma), \bar{\nabla}(\pi))=\delta_{\sigma, \pi}$.

Proof. We have head $\Delta(\sigma) \cong L(\sigma)$ and $\operatorname{soc} \bar{\nabla}(\pi) \cong L(\pi)$. So if $\pi \neq \sigma$ and $\operatorname{Hom}_{H}(\Delta(\sigma), \bar{\nabla}(\pi)) \neq 0$, we get $\sigma<\pi$ and $\pi \leq \sigma$, which is a contradiction.

Lemma 7.3. Let $\pi \in \Pi$. Then the module $\bar{\nabla}(\pi)$ has finite length; in particular, $\bar{\nabla}(\pi) \in H$-mod.

Proof. It suffices to prove that $\operatorname{Hom}_{H}(P(\sigma), \bar{\nabla}(\pi))$ is finite dimensional for every $\sigma \in \Pi$. But this follows from Lemma 7.2 and the fact that the multiplicity of $\Delta(\pi)$ in a $\Delta$-filtration of $P(\sigma)$ is finite, see Lemma 5.12 (iii).

Lemma 7.4. For any $\pi, \sigma \in \Pi$ and $i \geq 1$, we have $\operatorname{Ext}_{H}^{i}(\Delta(\sigma), \bar{\nabla}(\tau))=0$.

Proof. We apply induction on $i$. Let $i=1$. From the short exact sequence $0 \rightarrow \bar{\nabla}(\pi) \rightarrow I(\pi) \rightarrow I(\pi) / \bar{\nabla}(\pi) \rightarrow 0$ we get the exact sequence

$$
\begin{aligned}
0 & \rightarrow \operatorname{Hom}_{H}(\Delta(\sigma), \bar{\nabla}(\pi)) \rightarrow \operatorname{Hom}_{H}(\Delta(\sigma), I(\pi)) \rightarrow \operatorname{Hom}_{H}(\Delta(\sigma), I(\pi) / \bar{\nabla}(\pi)) \\
& \rightarrow \operatorname{Ext}_{H}^{1}(\Delta(\sigma), \bar{\nabla}(\pi)) \rightarrow 0 .
\end{aligned}
$$

By Lemma 5.10, $\operatorname{Ext}_{H}^{1}(\Delta(\sigma), L(\pi)) \neq 0$ implies $\pi>\sigma$. So Lemma 7.3 implies that $\operatorname{Ext}_{H}^{1}(\Delta(\sigma), \bar{\nabla}(\pi))=0$, unless $\pi>\sigma$. On the other hand, if $\pi>\sigma$, then by the definition of $\bar{\nabla}(\pi)$ we have $\operatorname{Hom}_{H}(\Delta(\sigma), I(\pi) / \bar{\nabla}(\pi))=0$, and so from the exact sequence above, we get $\operatorname{Ext}_{H}^{1}(\Delta(\sigma), \bar{\nabla}(\pi))=0$ again.

Let $i>1$ and suppose that we have proved that $\operatorname{Ext}_{H}^{i-1}(\Delta(\pi), \bar{\nabla}(\sigma))=0$ for all $\pi$ and $\sigma$. Applying $\operatorname{Hom}_{H}(-, \bar{\nabla}(\sigma))$ to the short exact sequence $0 \rightarrow$ 
$K(\pi) \rightarrow P(\pi) \rightarrow \Delta(\pi) \rightarrow 0$ and using the fact that $K(\pi)$ has a finite $\Delta$-filtration, completes the inductive step.

Lemma 7.5. If $V \in H$-mod has a $\Delta$-filtration, then

$$
(V: \Delta(\pi))_{q}=\operatorname{dim}_{q^{-1}} \operatorname{Hom}_{H}(V, \bar{\nabla}(\pi)) .
$$

Proof. Follows immediately from Lemmas 7.2 and 7.4 .

The following is a version of the Brauer-Humpreys-Bernstein-Gelfand-Gelfand Reciprocity:

Theorem 7.6. (BHBGG-Reciprocity) For any $\pi, \sigma \in \Pi$, we have

$$
(P(\pi): \Delta(\sigma))_{q}=[\bar{\nabla}(\sigma): L(\pi)]_{q^{-1}} .
$$

Proof. By Lemma 7.5, we have

$$
(P(\pi): \Delta(\sigma))_{q}=\operatorname{dim}_{q^{-1}} \operatorname{Hom}_{H}(P(\pi), \bar{\nabla}(\sigma))=[\bar{\nabla}(\sigma): L(\pi)]_{q^{-1}},
$$

as required.

Lemma 7.7. We have that $\operatorname{Ext}_{H}^{1}(L(\sigma), \bar{\nabla}(\pi))=0$ for all $\sigma<\pi$.

Proof. From the short exact sequence $0 \rightarrow \bar{\nabla}(\pi) \rightarrow I(\pi) \rightarrow I(\pi) / \bar{\nabla}(\pi) \rightarrow 0$ we get the exact sequence

$$
\begin{aligned}
0 & \rightarrow \operatorname{Hom}_{H}(L(\sigma), \bar{\nabla}(\pi)) \rightarrow \operatorname{Hom}_{H}(L(\sigma), I(\pi)) \rightarrow \operatorname{Hom}_{H}(L(\sigma), I(\pi) / \bar{\nabla}(\pi)) \\
& \rightarrow \operatorname{Ext}_{H}^{1}(L(\sigma), \bar{\nabla}(\pi)) \rightarrow 0,
\end{aligned}
$$

and the result follows since $\operatorname{Hom}_{H}(L(\sigma), I(\pi) / \bar{\nabla}(\pi))=0$.

7.2. Properties of good filtrations. We keep the notation of the previous subsection. In particular, $H$ is a $\mathscr{B}$-stratified algebra.

Lemma 7.8. Let $V \in H$-mod. Then the following are equivalent:

(i) $V$ has a $\Delta$-filtration;

(ii) $V$ has a finite $\Delta$-filtration;

(iii) $\operatorname{Ext}_{H}^{1}(V, \bar{\nabla}(\pi))=0$ for all $\pi \in \Pi$.

Proof. (i) $\Longleftrightarrow$ (ii) by Theorem 6.7 and Lemma 5.12(iii).

(ii) $\Longrightarrow$ (iii) is clear from Lemma 7.4.

(iii) $\Longrightarrow$ (ii) Let $\sigma$ be minimal with $\operatorname{Hom}_{H}(V, L(\sigma)) \neq 0$.

We claim that $\kappa \leq \sigma$ implies $\operatorname{Ext}_{H}^{1}(V, L(\kappa))=0$. Indeed, using the short exact sequence $0 \rightarrow L(\kappa) \rightarrow \bar{\nabla}(\kappa) \rightarrow \bar{\nabla}(\kappa) / L(\kappa) \rightarrow 0$, we get the exact sequence

$$
\operatorname{Hom}_{H}\left(V, \bar{\nabla}(\kappa) / L(\kappa) \rightarrow \operatorname{Ext}_{H}^{1}(V, L(\kappa)) \rightarrow \operatorname{Ext}_{H}^{1}(V, \bar{\nabla}(\kappa)),\right.
$$

which by assumptions implies the claim.

From $0 \rightarrow Q \rightarrow \Delta(\sigma) \rightarrow L(\sigma) \rightarrow 0$ we get the exact sequence

$$
\operatorname{Hom}_{H}(V, \Delta(\sigma)) \rightarrow \operatorname{Hom}_{H}(V, L(\sigma)) \rightarrow \operatorname{Ext}_{H}^{1}(V, Q) .
$$

By Lemma 3.8 and the claim proved in the previous paragraph, we deduce $\operatorname{Ext}_{H}^{1}(V, Q)=0$. Thus the map $\operatorname{Hom}_{H}(V, \Delta(\sigma)) \rightarrow \operatorname{Hom}_{H}(V, L(\sigma))$ is onto, and we deduce that there exists a surjective homomorphism $V \rightarrow q^{m} \Delta(\sigma)$ for some $m$, since head $\Delta(\sigma)=L(\sigma)$.

Thus we have found a submodule $V_{1} \subseteq V$ with $V / V_{1} \simeq \Delta(\sigma)$. For any $\pi$, we have an the exact sequence

$$
\operatorname{Ext}_{H}^{1}(V, \bar{\nabla}(\pi)) \rightarrow \operatorname{Ext}_{H}^{1}\left(V_{1}, \bar{\nabla}(\pi)\right) \rightarrow \operatorname{Ext}_{H}^{2}(\Delta(\sigma), \bar{\nabla}(\pi)),
$$


with the third term being zero by Lemma 7.4, so $V_{1}$ again satisfies the assumptions of the lemma. Continuing as above yields arbitrarily long filtration $V=V_{0} \supseteq$ $V_{1} \supseteq V_{2} \supseteq \cdots \supseteq V_{n}$ with quotients $\simeq \Delta(\pi)$ for various $\pi$, and $\operatorname{Ext}_{H}^{1}\left(V_{n}, \bar{\nabla}(\sigma)\right)=0$ for all $\sigma$. Now, by Lemma 7.4, for each $\sigma$, we have

$\operatorname{dim}_{q} \operatorname{Hom}_{H}(V, \bar{\nabla}(\sigma))=\sum_{i=0}^{n-1} \operatorname{dim}_{q} \operatorname{Hom}_{H}\left(V_{i} / V_{i+1}, \bar{\nabla}(\sigma)\right)+\operatorname{dim}_{q} \operatorname{Hom}_{H}\left(V_{n}, \bar{\nabla}(\sigma)\right)$.

Since $\Pi$ is finite and $\operatorname{Hom}_{H}(V, \bar{\nabla}(\sigma))$ is finite dimensional for all $\sigma \in \Pi$ by Lemma 7.3, the filtration must reach 0 for some $n$.

Lemma 7.9. Let $V$ be a finite length $H$-module. Then $V$ has a $\bar{\nabla}$-filtration if and only if $\operatorname{Ext}_{H}^{1}(\Delta(\pi), V)=0$ for all $\pi \in \Pi$.

Proof. The 'only-if' part is clear from Lemma 7.4, For the 'if-part', let $\sigma$ be minimal with $\operatorname{Hom}_{H}(L(\sigma), V) \neq 0$.

Let $\sigma \in \Pi$ be minimal with $\operatorname{Hom}_{H}(L(\sigma), V) \neq 0$. We claim that $\kappa<\sigma$ implies $\operatorname{Ext}_{H}^{1}(L(\kappa), V)=0$. Indeed, using $0 \rightarrow N \rightarrow \Delta(\kappa) \rightarrow L(\kappa) \rightarrow 0$, we get the exact sequence

$$
\operatorname{Hom}_{H}(N, V) \rightarrow \operatorname{Ext}_{H}^{1}(L(\kappa), V) \rightarrow \operatorname{Ext}_{H}^{1}(\Delta(\kappa), V),
$$

which by assumptions implies the claim.

From $0 \rightarrow L(\sigma) \rightarrow \bar{\nabla}(\sigma) \rightarrow Q \rightarrow 0$ we get the exact sequence

$$
\operatorname{Hom}_{H}(\bar{\nabla}(\sigma), V) \rightarrow \operatorname{Hom}_{H}(L(\sigma), V) \rightarrow \operatorname{Ext}_{H}^{1}(Q, V) .
$$

Using the claim from the previous paragraph, we deduce $\operatorname{Ext}_{H}^{1}(Q, V)=0$. So the $\operatorname{map} \operatorname{Hom}_{H}(\bar{\nabla}(\sigma), V) \rightarrow \operatorname{Hom}_{H}(L(\sigma), V)$ is onto. Since soc $\bar{\nabla}(\sigma)=L(\sigma)$, there exists an injective homomorphism $q^{m} \bar{\nabla}(\sigma) \rightarrow V$ for some $m \in \mathbb{Z}$.

Thus we have found a submodule $V_{1} \subseteq V$ with $V_{1} \simeq \bar{\nabla}(\sigma)$. For any $\pi$, we have an the exact sequence

$$
\operatorname{Ext}_{H}^{1}(\Delta(\pi), V) \rightarrow \operatorname{Ext}_{H}^{1}\left(\Delta(\pi), V / V_{1}\right) \rightarrow \operatorname{Ext}_{H}^{2}\left(\Delta(\pi), V_{1}\right),
$$

which implies, using Lemma 7.4, that $V / V_{1}$ again satisfies the assumptions of the lemma. Now we can apply induction on the length of $V$.

Corollary 7.10. Let $0 \rightarrow V^{\prime} \rightarrow V \rightarrow V^{\prime \prime} \rightarrow 0$ be a short exact sequence of $H$-modules.

(i) If $V$ and $V^{\prime \prime}$ have $\Delta$-filtrations, then so does $V^{\prime}$.

(ii) If $V$ and $V^{\prime}$ have finite $\bar{\nabla}$-filtrations, then so does $V^{\prime \prime}$.

Proof. Using Lemma 7.4 and long exact sequences in cohomology, (i) and (ii) follow from Lemmas 7.8 and 7.9 respectively.

Corollary 7.11. Let $W$ be a direct summand of an $H$-module $V$.

(i) If $V$ has a $\Delta$-filtration, then so does $W$.

(ii) If $V$ has a finite $\bar{\nabla}$-filtration, then so does $W$.

Proof. (i) and (ii) follow from Lemmas 7.8 and 7.9 respectively. 
7.3. Proper costandard objects in $\mathscr{B}$-highest weight categories. Let $\mathbf{C}$ be a $\mathscr{B}$-stratified category with respect to $(\Pi, \leq)$. Throughout this and the next subsection we make the additional assumption that $\Pi_{\leq \pi}$ is finite for every $\pi \in \Pi$.

Fix $\pi \in \Pi$. There exists a finite saturated set $\Sigma$ containing $\pi$-for example one can take $\Sigma=\Pi_{\leq \pi}$. By Proposition 5.16, the full subcategory $\mathbf{C}(\Sigma)$ in $\mathbf{C}$ is $\mathscr{B}$-stratified, with standard modules $\{\Delta(\sigma) \mid \sigma \in \Sigma\}$. By Corollary 6.8 there is a $\mathscr{B}$-stratified algebra $H_{\Sigma}$ such that the category $H_{\Sigma}$-mod is graded equivalent to $\mathbf{C}(\Sigma)$. Let $\bar{\nabla}_{\Sigma}(\pi)$ be the proper costandard $H_{\Sigma}$-module constructed in (7.1). Via equivalence between $H_{\Sigma}$-mod and $\mathbf{C}(\Sigma)$ we get an object of $\mathbf{C}(\Sigma)$, which we again denote $\bar{\nabla}_{\Sigma}(\pi)$. Since $\mathbf{C}(\Sigma)$ is a subcategory of $\mathbf{C}$, we may consider $\bar{\nabla}_{\Sigma}(\pi)$ as an object of $\mathbf{C}$. The following lemma shows that this construction does not depend on the choice of $\Sigma$ :

Lemma 7.12. If $\Sigma, \Omega \subseteq \Pi$ are finite saturated subsets containing $\pi \in \Pi$, then $\bar{\nabla}_{\Sigma}(\pi) \cong \bar{\nabla}_{\Omega}(\pi)$.

Proof. By passing to $\Sigma \cup \Omega$, we may assume that $\Sigma \subseteq \Omega$. By Corollary 6.8, there is a $\mathscr{B}$-stratified algebra $H_{\Sigma}$ with $H_{\Sigma}$-mod graded equivalent to $\mathbf{C}(\Sigma)$ and a $\mathscr{B}$-stratified algebra $H_{\Omega}$ with $H_{\Omega}$-mod graded equivalent to $\mathbf{C}(\Omega)$. Moreover, $\mathbf{C}(\Sigma)=(\mathbf{C}(\Omega))(\Sigma)$, and, using the notation (3.11), we also have $H_{\Sigma} \cong H_{\Omega}(\Sigma)$.

By definition, the composition factors of the modules $\bar{\nabla}_{\Sigma}(\pi)$ and $\bar{\nabla}_{\Omega}(\pi)$ are of the form $L(\sigma)$ for $\sigma \leq \pi$, in particular $\sigma \in \Sigma$. It follows that $\mathcal{Q}^{\Sigma}\left(\bar{\nabla}_{\Omega}(\pi)\right)=$ $\bar{\nabla}_{\Omega}(\pi)$, and so both $\bar{\nabla}_{\Sigma}(\pi)$ and $\bar{\nabla}_{\Omega}(\pi)$ can be considered as modules over $H_{\Sigma}$. The short exact sequence of $H_{\Sigma}$-modules $0 \rightarrow L(\pi) \rightarrow \bar{\nabla}_{\Omega}(\pi) \rightarrow \bar{\nabla}_{\Omega}(\pi) / L(\pi) \rightarrow 0$ yields the long exact sequence

$$
\begin{aligned}
0 & \rightarrow \operatorname{Hom}_{H_{\Sigma}}\left(\bar{\nabla}_{\Omega}(\pi) / L(\pi), \bar{\nabla}_{\Sigma}(\pi)\right) \rightarrow \operatorname{Hom}_{H_{\Sigma}}\left(\bar{\nabla}_{\Omega}(\pi), \bar{\nabla}_{\Sigma}(\pi)\right) \\
& \rightarrow \operatorname{Hom}_{H_{\Sigma}}\left(L(\pi), \bar{\nabla}_{\Sigma}(\pi)\right) \rightarrow \operatorname{Ext}_{H_{\Sigma}}^{1}\left(\bar{\nabla}_{\Omega}(\pi) / L(\pi), \bar{\nabla}_{\Sigma}(\pi)\right) .
\end{aligned}
$$

Note that $\operatorname{Hom}_{H_{\Sigma}}\left(\bar{\nabla}_{\Omega}(\pi) / L(\pi), \bar{\nabla}_{\Sigma}(\pi)\right)=0$, since all composition factors of $\bar{\nabla}_{\Omega}(\pi) / L(\pi)$ are of the form $L(\sigma)$ for $\sigma<\pi$, and soc $\bar{\nabla}_{\Sigma}(\pi) \cong L(\pi)$. Moreover, $\operatorname{Ext}_{H_{\Sigma}}^{1}\left(\bar{\nabla}_{\Omega}(\pi) / L(\pi), \bar{\nabla}_{\Sigma}(\pi)\right)=0$ by Lemma 7.7. It follows that the embedding soc $\bar{\nabla}_{\Omega}(\pi)=L(\pi) \hookrightarrow \bar{\nabla}_{\Sigma}(\pi)$ lifts to a map $\bar{\nabla}_{\Omega}(\pi) \rightarrow \bar{\nabla}_{\Sigma}(\pi)$, which has to be injective. Similarly, $\bar{\nabla}_{\Sigma}(\pi)$ embeds into $\bar{\nabla}_{\Omega}(\pi)$. As both are finite length modules by Lemma 7.3 , the result follows.

In view of the lemma, we can drop the index $\Sigma$ from the notation $\bar{\nabla}_{\Sigma}(\pi)$ and simply write $\bar{\nabla}(\pi) \in \mathbf{C}$. This is a proper costandard object in $\mathbf{C}$.

Theorem 7.13. Let $\mathbf{C}$ be a $\mathscr{B}$-stratified category. Assume that $\Pi_{\leq \pi}$ is finite for all $\pi \in \Pi$. Fix $\pi, \sigma \in \Pi$. Then:

(i) The object $\bar{\nabla}(\pi) \in \mathbf{C}$ has finite length, soc $\bar{\nabla}(\pi) \cong L(\pi)$, and all composition factors of $\bar{\nabla}(\pi) / \operatorname{soc} \bar{\nabla}(\pi)$ are of the form $L(\kappa)$ for $\kappa<\pi$.

(ii) We have

and

$$
\operatorname{dim}_{q} \operatorname{Hom}_{\mathbf{C}}(\Delta(\sigma), \bar{\nabla}(\pi))=\delta_{\sigma, \pi}
$$

$$
\operatorname{Ext}_{\mathbf{C}}^{1}(\Delta(\sigma), \bar{\nabla}(\pi))=0 .
$$

(iii) If $\sigma<\pi$, then $\operatorname{Ext}_{\mathbf{C}}^{1}(L(\sigma), \bar{\nabla}(\pi))=0$.

(iv) If $V \in \mathbf{C}$ has a $\Delta$-filtration, then $(V: \Delta(\pi))_{q}=\operatorname{dim}_{q^{-1}} \operatorname{Hom}_{\mathbf{C}}(V, \bar{\nabla}(\pi))$.

(v) We have $(P(\pi): \Delta(\sigma))_{q}=[\bar{\nabla}(\sigma): L(\pi)]_{q^{-1}}$. 
Proof. Let $\Sigma$ be a finite saturated set containing $\sigma$ and $\pi$, for example, we can take $\Sigma=\Pi_{\leq \sigma} \cup \Pi_{\leq \pi}$. Then the category $\mathbf{C}(\Sigma)$ is $\mathscr{B}$-stratified, and by Corollary 6.8 , it is graded equivalent to $H_{\Sigma}$-mod for some $\mathscr{B}$-stratified algebra $H_{\Sigma}$. So we have (i)(v) holding in $\mathbf{C}(\Sigma)$ by the corresponding facts in $H_{\Sigma}$-mod proved in $\$ 7.1$. Since $\mathbf{C}(\Sigma)$ is a full subcategory, and in view of Lemma 5.14 and Proposition 5.16, part (i), the first equality in part (ii), and parts (iv) and (v) follow. To prove the facts involving Ext $^{1}$, it now remains to note that any extension in $\mathbf{C}$ of $\bar{\nabla}(\pi)$ by $\Delta(\sigma)$ or by $L(\sigma)$ belongs to $\mathbf{C}(\Sigma)$.

7.4. Good filtrations in $\mathscr{B}$-stratified categories. Throughout the subsection, $\mathbf{C}$ is a $\mathscr{B}$-stratified category such that $\Pi$ is countable and $\Pi_{\leq \pi}$ is finite for every $\pi \in \Pi$. These assumptions are equivalent to the fact that there is a nested family of finite saturated sets

$$
\Sigma_{1} \subseteq \Sigma_{2} \subseteq \ldots \text { with } \quad \cup_{n \geq 1} \Sigma_{n}=\Pi .
$$

Lemma 7.15. Let $0 \rightarrow U \rightarrow V \rightarrow W \rightarrow 0$ be a short exact sequence in $\mathbf{C}$. If $V$ and $W$ have $\Delta$-filtrations, then so does $U$, and $(U: \Delta(\pi))_{q}+(W: \Delta(\pi))_{q}=$ $(V: \Delta(\pi))_{q}$ for all $\pi \in \Pi$.

Proof. For any finite saturated $\Sigma \subseteq \Pi$, we have a short exact sequence

$$
0 \rightarrow U \cap O^{\Sigma}(V) \rightarrow V / O^{\Sigma}(V) \rightarrow W / O^{\Sigma}(W) \rightarrow 0
$$

By Lemmas 5.14 and $5.12\left(\right.$ iii), $V / O^{\Sigma}(V)$ and $W / O^{\Sigma}(W)$ have finite $\Delta$-filtrations. The category $\mathbf{C}(\Sigma)$ is $\mathscr{B}$-stratified, and by Corollary 6.8, it is graded equivalent to $H_{\Sigma}$-mod for some $\mathscr{B}$-stratified algebra $H_{\Sigma}$. So $U \cap O^{\Sigma}(V)$ has a finite $\Delta$-filtration by Corollary 7.10 (i).

Using the family (7.14), we get an exhaustive filtration

$$
U \supseteq U \cap O^{\Sigma_{1}}(V) \supseteq U \cap O^{\Sigma_{2}}(V) \supseteq \ldots
$$

whose subfactors have finite $\Delta$-filtrations by Lemmas 5.14 and 5.12 (iii). It follows that $U$ has a $\Delta$-filtration. Now, the statement about the multiplicities is clear.

Lemma 7.16. Let $V \in \mathbf{C}$ have a $\Delta$-filtration, $M:=\left\{\sigma \in \Pi \mid(V: \Delta(\sigma))_{q} \neq 0\right\}$, and $\Pi_{\geq M}=\{\tau \in \Pi \mid \tau \geq \sigma$ for some $\sigma \in M\}$. Then $V$ has projective resolution $\cdots \rightarrow P_{1} \rightarrow P_{0} \rightarrow V$, where each $P_{i}$ is a finite direct sum of projectives of the form $q^{m} P(\tau)$ with $\tau \in \Pi_{\geq M}$.

Proof. By Lemma 4.7, there is a module $P_{0}$ of the required form and an epimorphism $P_{0} \rightarrow V$. By Lemma 7.15 and (SC1), we have a short exact sequence

$$
0 \rightarrow K \rightarrow P_{0} \rightarrow V \rightarrow 0,
$$

where $K$ has $\Delta$-filtration with subfactors $\simeq \Delta(\tau)$ for $\tau \in \Pi_{\geq M}$. Now, there is a module $P_{1}$ of the required form and an epimorphism $P_{1} \rightarrow \bar{K}$, and so on.

Lemma 7.17. Let $V \in \mathbf{C}$, and $\Sigma_{1} \subset \Sigma_{2} \subset \ldots$ be finite saturated subsets of $\Pi$ with $\Pi=\cup_{n \geq 1} \Sigma_{n}$. If $\mathcal{Q}^{\Sigma_{n}}(V)$ has a $\Delta$-filtration for every $n$, then so does $V$.

Proof. We have the sequence $V \supseteq \mathcal{O}^{\Sigma_{1}}(V) \supseteq \mathcal{O}^{\Sigma_{2}} \supseteq \ldots$ with $\cap_{n \geq 1} \mathcal{O}^{\Sigma_{n}}(V)=$ (0), so it suffice to show that each $\mathcal{O}^{\Sigma_{n}}(V) / \mathcal{O}^{\Sigma_{n+1}}(V)$ has a finite $\Delta$-filtration. By Proposition [5.16, $\mathbf{C}\left(\Sigma_{n+1}\right)$ is a $\mathscr{B}$-stratified category. By assumption and Lemma 5.12 (iii), we have that $V / \mathcal{O}^{\Sigma_{n+1}}(V)=\mathcal{Q}^{\Sigma_{n+1}}(V) \in \mathbf{C}\left(\Sigma_{n+1}\right)$ has a finite $\Delta$-filtration. Now apply Corollary 7.10 (i). 
We denote by $L_{i} \mathcal{Q}^{\Sigma}$ the $i$ th left derived functor of the functor $\mathcal{Q}^{\Sigma}: \mathbf{C} \rightarrow \mathbf{C}(\Sigma)$.

Lemma 7.18. Let $V \in \mathbf{C}$ have a $\Delta$-filtration, and $\Sigma \subseteq \Pi$ be a finite saturated subset. Then $L_{i} \mathcal{Q}^{\Sigma}(V)=0$ for all $i>0$.

Proof. Let $\pi \in \Pi$. From the exact sequence $0 \rightarrow K(\pi) \rightarrow P(\pi) \rightarrow \Delta(\pi) \rightarrow 0$ we get the exact sequence

$$
0 \rightarrow L_{1} \mathcal{Q}^{\Sigma}(\Delta(\pi)) \rightarrow \mathcal{Q}^{\Sigma}(K(\pi)) \rightarrow \mathcal{Q}^{\Sigma}(P(\pi)) \rightarrow \mathcal{Q}^{\Sigma}(\Delta(\pi)) \rightarrow 0
$$

and the isomorphisms $L_{i+1} \mathcal{Q}^{\Sigma}(\Delta(\pi)) \cong L_{i} \mathcal{Q}^{\Sigma}(K(\pi))$.

If $\pi \in \Sigma$, then $\mathcal{O}^{\Sigma}(K(\pi))=\mathcal{O}^{\Sigma}(P(\pi))$, so the map $\mathcal{Q}^{\Sigma}(K(\pi)) \rightarrow \mathcal{Q}^{\Sigma}(P(\pi))$ is injective, whence $L_{1} \mathcal{Q}^{\Sigma}(\Delta(\pi))=0$. If $\pi \notin \Sigma$, then no $\sigma>\pi$ belongs to $\Sigma$, whence $\mathcal{Q}^{\Sigma}(K(\pi))=0$ in view of (SC1), and again $L_{1} \mathcal{Q}^{\Sigma}(\Delta(\pi))=0$. We have proved that $L_{1} \mathcal{Q}^{\Sigma}(\Delta(\pi))=0$ for all $\pi$, hence $L_{1} \mathcal{Q}^{\Sigma}(V)=0$ for any $V$, which has a finite $\Delta$-filtration, by the long exact sequence argument.

To deal with an arbitrary $\Delta$-filtration, note by Lemma 5.14 that $\mathcal{O}^{\Sigma}(V)$ has a $\Delta$-filtration with subfactors $\simeq \Delta(\tau)$ for $\tau \in \Pi \backslash \Sigma$. So by Lemma 17.16, there is a projective resolution $\cdots \rightarrow P_{1} \rightarrow P_{0} \rightarrow \mathcal{O}^{\Sigma}(V)$, where each $P_{i}$ is a finite direct sum of projectives of the form $q^{m} P(\tau)$ with $\tau \in \Pi \backslash \Sigma$. Hence $\mathcal{Q}^{\Sigma}\left(P_{i}\right)=0$ for all $i>0$, and we have $L_{i} \mathcal{Q}^{\Sigma}\left(\mathcal{O}^{\Sigma}(V)\right)=0$ for all $i>0$. So, since $\mathcal{Q}^{\Sigma}(V)$ has a finite $\Delta$-filtration, the long exact sequence corresponding to $0 \rightarrow \mathcal{O}^{\Sigma}(V) \rightarrow V \rightarrow$ $\mathcal{Q}^{\Sigma}(V) \rightarrow 0$ gives $L_{i} \mathcal{Q}^{\Sigma}(V)=0$.

Now, by the first paragraph, $L_{2} \mathcal{Q}^{\Sigma}(\Delta(\pi)) \cong L_{1} \mathcal{Q}^{\Sigma}(K(\pi))=0$. Therefore $L_{2} \mathcal{Q}^{\Sigma}(V)=0$ for all modules $V$ with finite $\Delta$-filtration, and then as in the second paragraph one shows that $L_{2} \mathcal{Q}^{\Sigma}(V)=0$ for modules $V$ with an arbitrary $\Delta$-filtration. Continuing like this we prove $L_{i} \mathcal{Q}^{\Sigma}(V)=0$ for all $i>0$.

Lemma 7.19. If $\Sigma \subseteq \Pi$ is a finite saturated subset and $V \in \mathbf{C}(\Sigma)$, then $L_{i} \mathcal{Q}^{\Sigma}(V)=0$ for all $i>0$.

Proof. Note that $V$ is a quotient of a module $P$ which is a finite direct sum of modules $\simeq P(\sigma)$ with $\sigma \in \Sigma$. So $V$ is also a quotient of $\mathcal{Q}^{\Sigma}(P)$ which has a $\Delta$-filtration by Lemma 5.14. Hence $L_{i} \mathcal{Q}^{\Sigma}\left(\mathcal{Q}^{\Sigma}(P)\right)=0$ by Lemma 7.18. So the short exact sequence $0 \rightarrow N \rightarrow \mathcal{Q}^{\Sigma}(P) \rightarrow V \rightarrow 0$ yields an exact sequence

$$
0 \rightarrow L_{1} \mathcal{Q}^{\Sigma}(V) \rightarrow N \rightarrow \mathcal{Q}^{\Sigma}(P) \rightarrow V \rightarrow 0
$$

and isomorphisms $L_{i+1} \mathcal{Q}^{\Sigma}(V) \cong L_{i} \mathcal{Q}^{\Sigma}(N)$ for all $i>0$. The exact sequence implies that $L_{1} \mathcal{Q}^{\Sigma}(V)=0$ for all $\pi \in \Sigma$. Since $V$ is an arbitrary object in $\mathbf{C}(\Sigma)$, we now deduce that $L_{1} \mathcal{Q}^{\Sigma}(N)$. Then $L_{2} \mathcal{Q}^{\Sigma}(V)=0$, and so on by induction.

Proposition 7.20. Let $\Sigma \subseteq \Pi$ be a finite saturated subset. If $V, W \in \mathbf{C}(\Sigma)$, then $\operatorname{Ext}_{\mathbf{C}(\Sigma)}^{i}(V, W) \cong \operatorname{Ext}_{\mathbf{C}}^{i}(V, W)$ for all $i \geq 0$.

Proof. The functor $\mathcal{Q}^{\Sigma}$ takes projectives to acyclics, so we have the Grothendieck spectral sequence [44, Theorem 10.48]:

$$
\operatorname{Ext}_{\mathbf{C}(\Sigma)}^{i}\left(L_{j} \mathcal{Q}^{\Sigma}(W), V\right) \Longrightarrow \operatorname{Ext}_{\mathbf{C}}^{i+j}(W, V) .
$$

But $L_{j} \mathcal{Q}^{\Sigma}(W)=0$ for $j>0$ by Lemma 7.19, so the spectral sequence degenerates, and we get the required isomorphism.

Theorem 7.21. Let $\mathbf{C}$ be a $\mathscr{B}$-stratified category with countable $\Pi$ such that $\Pi_{\leq \pi}$ is finite for every $\pi \in \Pi$. Then $V \in \mathbf{C}$ has a $\Delta$-filtration if and only 
if $\operatorname{Ext}_{\mathbf{C}}^{1}(V, \bar{\nabla}(\pi))=0$ for all $\pi \in \Pi$. Moreover, if $V$ has a $\Delta$-filtration then $\operatorname{Ext}_{\mathbf{C}}^{i}(V, \bar{\nabla}(\pi))=0$ for all $\pi \in \Pi$ and $i>0$.

Proof. Let $\Sigma_{n}$ be as in (7.14). For each $n$, the category $\mathbf{C}\left(\Sigma_{n}\right)$ is $\mathscr{B}$-stratified, so by Corollary 6.8, it is graded equivalent to $H_{\Sigma_{n}}$-mod for some $\mathscr{B}$-stratified algebra $H_{\Sigma_{n}}$. Hence the statement of the theorem holds in each $\mathbf{C}\left(\Sigma_{n}\right)$ by Lemmas 7.8 and 7.4 .

Assume that $\operatorname{Ext}_{\mathbf{C}}^{1}(V, \bar{\nabla}(\pi))=0$ for all $\pi$. Chose $n$ with $\pi \in \Sigma_{n}$. From the exact sequence $0 \rightarrow \mathcal{O}^{\Sigma_{n}}(V) \rightarrow V \rightarrow \mathcal{Q}^{\Sigma_{n}}(V) \rightarrow 0$ we get the exact sequence

$$
\begin{aligned}
0 & \rightarrow \operatorname{Hom}_{\mathbf{C}}\left(\mathcal{Q}^{\Sigma_{n}}(V), \bar{\nabla}(\pi)\right) \rightarrow \operatorname{Hom}_{\mathbf{C}}(V, \bar{\nabla}(\pi)) \rightarrow \operatorname{Hom}_{\mathbf{C}}\left(\mathcal{O}^{\Sigma_{n}}(V), \bar{\nabla}(\pi)\right) \\
& \rightarrow \operatorname{Ext}_{\mathbf{C}}^{1}\left(\mathcal{Q}^{\Sigma_{n}}(V), \bar{\nabla}(\pi)\right) \rightarrow 0 .
\end{aligned}
$$

Note that $\operatorname{Hom}_{\mathbf{C}}\left(\mathcal{O}^{\Sigma_{n}}(V), \bar{\nabla}(\pi)\right)=0$, and so $\operatorname{Ext}_{\mathbf{C}}^{1}\left(\mathcal{Q}^{\Sigma_{n}}(V), \bar{\nabla}(\pi)\right)=0$, whence $\operatorname{Ext}_{\mathbf{C}\left(\Sigma_{n}\right)}^{1}\left(\mathcal{Q}^{\Sigma_{n}}(V), \bar{\nabla}(\pi)\right)=0$. By the first paragraph, $\mathcal{Q}^{\Sigma_{n}}(V)$ has a finite $\Delta$ filtration. Now apply Lemma 7.17 to deduce that $V$ has a $\Delta$-filtration.

Conversely, assume that $V$ has a $\Delta$-filtration. Fix $\pi$ and pick $n$ so that $\pi \in \Sigma_{n}$. By Lemma 5.14 that $\mathcal{O}^{\Sigma_{n}}(V)$ and $\mathcal{Q}^{\Sigma_{n}}(V)$ have $\Delta$-filtrations. By Proposition 7.20, Lemma 7.4, and the long exact sequence in cohomology, we have $\operatorname{Ext}_{\mathbf{C}}^{i}\left(\mathcal{Q}^{\Sigma_{n}}(V), \bar{\nabla}(\pi)\right)=0$ for all $i>0$. On the other hand, by Lemma 7.16. $\mathcal{O}^{\Sigma_{n}}(V)$ has a projective resolution of the form $\ldots P_{1} \rightarrow P_{0} \rightarrow \mathcal{O}^{\Sigma_{n}}(V)$, where each $P_{i}$ is a finite direct sum of projectives of the form $q^{m} P(\tau)$ with $\tau \notin \Sigma_{n}$. So $\operatorname{Hom}_{\mathbf{C}}\left(P_{i}, \bar{\nabla}(\pi)\right)=0$, and in particular, $\operatorname{Ext}_{\mathbf{C}}^{i}\left(\mathcal{O}^{\Sigma_{n}}(V), \bar{\nabla}(\pi)\right)=0$. From the long exact sequence in cohomology, we now deduce $\operatorname{Ext}_{\mathbf{C}}^{i}(V, \bar{\nabla}(\pi))=0$.

Lemma 7.22. A finite length object $V \in \mathbf{C}$ has a $\bar{\nabla}$-filtration if and only if $\operatorname{Ext}_{\mathbf{C}}^{1}(\Delta(\pi), V)=0$ for all $\pi \in \Pi$.

Proof. The 'only-if' part is clear from Theorem 7.13(ii). For the 'if-part', work in $\mathbf{C}(\Sigma)$ for sufficiently large finite saturated subset $\Sigma \subset \Pi$ and apply Lemma 7.9 and Proposition 7.20 .

Corollary 7.23. Let $0 \rightarrow V^{\prime} \rightarrow V \rightarrow V^{\prime \prime} \rightarrow 0$ be a short exact sequence in $\mathbf{C}$.

(i) If $V$ and $V^{\prime \prime}$ have $\Delta$-filtrations, then so does $V^{\prime}$.

(ii) If $V$ and $V^{\prime}$ have finite $\bar{\nabla}$-filtrations, then so does $V^{\prime \prime}$.

Proof. Similar to the proof of Corollary 7.10 .

Corollary 7.24. Let $W$ be a direct summand of $V \in \mathbf{C}$.

(i) If $V$ has a $\Delta$-filtration, then so does $W$.

(ii) If $V$ has a finite $\bar{\nabla}$-filtration, then so does $W$.

Proof. Similar to the proof of Corollary 7.11 .

\section{8. $\mathscr{B}$-analogue of the Dlab-Ringel Standardization Theorem}

In this section, we generalize results of [18, §3]. Throughout the section, $\mathbf{C}$ is a graded abelian $F$-linear category, and $\mathscr{B}$ is a fixed class of connected algebras.

8.1. Standardizing families. Let $\Theta=\{\Theta(\pi) \mid \pi \in \Pi\}$ be a family of objects of $\mathbf{C}$ labeled by a finite partially ordered set $\Pi$. The family $\Theta$ is called weakly $\mathscr{B}$-standardizing if the following conditions are satisfied:

(End) For each $\pi \in \Pi$, we have that $B_{\pi}:=\operatorname{End}(\Theta(\pi))^{\text {op }}$ belongs to $\mathscr{B}$. 
(FG) For each $\pi, \sigma \in \Pi$, we have that the $B_{\sigma}$-modules $\operatorname{Hom}(\Theta(\pi), \Theta(\sigma))$ and $\operatorname{Ext}^{1}(\Theta(\pi), \Theta(\sigma))$ are finitely generated.

(Dir) $\operatorname{Hom}(\Theta(\pi), \Theta(\sigma)) \neq 0$ implies $\pi \leq \sigma$, and $\operatorname{Ext}^{1}(\Theta(\pi), \Theta(\sigma)) \neq 0$ implies $\pi<\sigma$.

Let $\Theta$ be a weakly $\mathscr{B}$-standardizing family. The condition (End) implies that the objects $\Theta(\pi)$ are indecomposable. Denote by $\operatorname{Fil}(\Theta)$ the full (graded) subcategory of $\mathbf{C}$ of all objects in $\mathbf{C}$ having finite $\Theta$-filtrations, i.e. a finite filtration with subquotients $\simeq \Theta(\pi)$ for $\pi \in \Pi$.

If $V \in \operatorname{Fil}(\Theta)$ and $\sigma \in \Pi$. We denote by $(V: \Theta(\sigma))_{q}$ the Laurent polynomial $m_{\sigma}(q)=\sum_{n \in \mathbb{Z}} m_{n} q^{n}$, where $m_{n}$ is the number of times $q^{n} \Theta(\sigma)$ appears as a subquotient in some $\Theta$-filtration of $V$. This number does not depend on the choice of the $\Theta$-filtration. Indeed, let $V=V_{N} \supset \cdots \supset V_{1} \supset V_{0}=(0)$ be a $\Theta$-filtration, and $\pi$ be a maximal element such that $m_{\pi}(q) \neq 0$. The Ext-condition from (Dir) shows that we can choose another filtration $V=V_{M}^{\prime} \supset \cdots \supset V_{1}^{\prime} \supset V_{0}^{\prime}=(0)$ such that $V_{1}^{\prime} \cong m_{\pi}(q) \Theta(\pi)$, and all other subquotient are of the form $q^{n} \Theta(\sigma)$ for $\sigma \neq \pi$ appearing with the same multiplicities as in the original filtration. Now, we can recover $m_{\pi}(q)$ as the (graded) rank of the free $B_{\pi}$-module $\operatorname{Hom}(\Theta(\pi), V)$. Then we pass to $V / V_{1}^{\prime}$ and repeat.

The main example of a weakly $\mathscr{B}$-standardizing family is as follows. Let $\mathbf{C}$ be a weakly $\mathscr{B}$-highest weight category with poset $\Sigma$, and $\Pi \subseteq \Sigma$ be a finite subset with partial order induced from that on $\Sigma$. Then $\Delta:=\{\Delta(\pi) \mid \pi \in \Pi\}$ is a weakly $\mathscr{B}$-standardizing family. Indeed, the property (End) holds by definition, and the property (Dir) comes from Lemmas 4.3 and 5.10(ii). Finally, the property (FG) is contained in the following:

Lemma 8.1. If $\mathbf{C}$ is a weakly $\mathscr{B}$-highest weight category, $V \in \mathbf{C}$, and $\pi \in \Pi$, then the $B_{\pi}$-modules $\operatorname{Ext}^{i}(V, \Delta(\pi))$ are finitely generated for all $i \geq 0$.

Proof. This comes from Corollary 3.4(i) and (FGen).

8.2. Standardization Theorem. In the previous subsection we have noted that a finite family $\Delta:=\{\Delta(\pi) \mid \pi \in \Pi\}$ of standard modules in a weakly $\mathscr{B}$-highest weight category is a weakly $\mathscr{B}$-standardizing family. The goal of this subsection is to prove a converse statement in some sense, see Theorem 8.3 below.

Lemma 8.2. Let $\Theta=\{\Theta(\pi) \mid \pi \in \Pi\}$ be a weakly $\mathscr{B}$-standardizing family in a graded abelian F-linear category $\mathbf{C}$. Then:

(i) for each $\pi \in \Pi$, there exists an indecomposable object $P_{\Theta}(\pi) \in \mathrm{Fil}(\Theta)$ and an epimorphism $P_{\Theta}(\pi) \rightarrow \Theta(\pi)$ with kernel in $\mathrm{Fil}(\Theta)$ and such that $\operatorname{Ext}^{1}\left(P_{\Theta}(\pi), V\right)=0$ for all $V \in \mathrm{Fil}(\Theta)$.

(ii) For any object $X \in \mathrm{Fil}(\Theta)$, there exists an exact sequence

$$
0 \rightarrow X^{\prime} \rightarrow P_{0}(X) \rightarrow X \rightarrow 0
$$

where $P_{0}(X)$ is a finite direct sum of objects $\simeq P_{\Theta}(\pi)$, and $X^{\prime} \in \mathrm{Fil}(\Theta)$.

Proof. (i) If $\pi$ is a maximal element in $\Pi$, we can take $P_{\Theta}(\pi)=\Theta(\pi)$. Otherwise, let $\sigma \in \Pi$ be minimal with $\sigma>\pi$. Let $\xi_{1}, \ldots, \xi_{r}$ be a minimal set of generators of the $B_{\sigma}$-module $\operatorname{Ext}^{1}(\Theta(\pi), \Theta(\sigma))_{B_{\sigma}}$, and $0 \rightarrow \Theta(\sigma) \rightarrow E_{1} \rightarrow \Theta(\pi) \rightarrow 0$ be the 
extension corresponding to $\xi_{1}$. It yields the long exact sequence

$$
\begin{aligned}
0 & \rightarrow \operatorname{Hom}(\Theta(\pi), \Theta(\sigma))_{B_{\sigma}} \rightarrow \operatorname{Hom}\left(E_{1}, \Theta(\sigma)\right)_{B_{\sigma}} \stackrel{\chi}{\longrightarrow} \operatorname{Hom}(\Theta(\sigma), \Theta(\sigma))_{B_{\sigma}} \\
& \stackrel{\varphi}{\longrightarrow} \operatorname{Ext}^{1}(\Theta(\pi), \Theta(\sigma))_{B_{\sigma}} \stackrel{\psi}{\longrightarrow} \operatorname{Ext}^{1}\left(E_{1}, \Theta(\sigma)\right)_{B_{\sigma}} \rightarrow 0 .
\end{aligned}
$$

The connecting homomorphism $\varphi$ maps the identity map $\operatorname{id}_{\Theta(\sigma)}$ to $\xi_{1}$. It follows that $\operatorname{Ext}^{1}\left(E_{1}, \Theta(\sigma)\right)_{B_{\sigma}}$ is generated by $\bar{\xi}_{2}:=\psi\left(\xi_{2}\right), \ldots, \bar{\xi}_{r}:=\psi\left(\xi_{r}\right)$ as a (right) $B_{\sigma}$-module. In fact, note that $\operatorname{Ext}^{1}\left(E_{1}, \Theta(\sigma)\right)_{B_{\sigma}} \simeq \operatorname{Ext}^{1}(\Theta(\pi), \Theta(\sigma))_{B_{\sigma}} /\left(\xi_{1} \cdot B_{\sigma}\right)$. Finally, $E_{1}$ is indecomposable since $\varphi \neq 0$, and so $\chi$ is not onto.

Let $0 \rightarrow \Theta(\sigma) \rightarrow E_{2} \rightarrow E_{1} \rightarrow 0$ be the extension corresponding to $\bar{\xi}_{2} \in$ $\operatorname{Ext}^{1}\left(E_{1}, \Theta(\sigma)\right)$. It yields the long exact sequence

$$
\begin{aligned}
0 & \rightarrow \operatorname{Hom}\left(E_{1}, \Theta(\sigma)\right)_{B_{\sigma}} \rightarrow \operatorname{Hom}\left(E_{2}, \Theta(\sigma)\right)_{B_{\sigma}} \stackrel{\chi}{\longrightarrow} \operatorname{Hom}(\Theta(\sigma), \Theta(\sigma))_{B_{\sigma}} \\
& \stackrel{\varphi}{\longrightarrow} \operatorname{Ext}^{1}\left(E_{1}, \Theta(\sigma)\right)_{B_{\sigma}} \stackrel{\psi}{\longrightarrow} \operatorname{Ext}^{1}\left(E_{2}, \Theta(\sigma)\right)_{B_{\sigma}} \rightarrow 0 .
\end{aligned}
$$

The connecting homomorphism $\varphi$ maps the identity map $\operatorname{id}_{\Theta(\sigma)}$ to $\bar{\xi}_{2}$. It follows that $\operatorname{Ext}^{1}\left(E_{2}, \Theta(\sigma)\right)_{B_{\sigma}}$ is generated by $\psi\left(\bar{\xi}_{3}\right), \ldots, \psi\left(\bar{\xi}_{r}\right)$ as a $B_{\sigma}$-module. In fact, $\operatorname{Ext}^{1}\left(E_{2}, \Theta(\sigma)\right)_{B_{\sigma}} \simeq \operatorname{Ext}^{1}(\Theta(\pi), \Theta(\sigma))_{B_{\sigma}} /\left(\xi_{1} \cdot B_{\sigma}+\xi_{2} \cdot B_{\sigma}\right)$. Finally, $E_{2}$ is an indecomposable object in $\mathrm{Fil}(\Theta)$ since $\varphi$ is non-zero, and so $\chi$ is not onto. Continuing this way with $\xi_{3}, \ldots, \xi_{r}$, we get an indecomposable object $E(\pi, \sigma) \in \mathrm{Fil}(\Theta)$ such that there exists an exact sequence

$$
0 \rightarrow\left(\operatorname{rank}_{q} \operatorname{Ext}^{1}(\Theta(\pi), \Theta(\sigma))_{B_{\sigma}}\right) \cdot \Theta(\sigma) \rightarrow E(\pi, \sigma) \rightarrow \Theta(\pi) \rightarrow 0
$$

and

$$
\operatorname{Ext}^{1}(E(\pi, \sigma), \Theta(\sigma))=\operatorname{Ext}^{1}(E(\pi, \sigma), \Theta(\pi))=0 .
$$

If $\pi$ is a maximal element of $\Pi \backslash\{\sigma\}$, we can take $P_{\Theta}(\pi)=E(\pi, \sigma)$. Otherwise, let $\kappa \in \Pi \backslash\{\sigma\}$ be a minimal with $\kappa>\pi$. As above, we construct an extension $E(\pi, \sigma, \kappa)$ of $E(\pi, \sigma)$ by $\left(\operatorname{rank}_{q} \operatorname{Ext}^{1}(E(\pi, \sigma), \Theta(\kappa))_{B_{\sigma}}\right) \cdot \Theta(\kappa)$ such that

$$
\operatorname{Ext}^{1}(E(\pi, \sigma, \kappa), \Theta(\pi))=\operatorname{Ext}^{1}(E(\pi, \sigma, \kappa), \Theta(\sigma))=\operatorname{Ext}^{1}(E(\pi, \sigma, \kappa), \Theta(\kappa))=0 .
$$

The process will stop after finitely many steps to produce $P_{\Theta}(\pi)$, since $\Pi$ is finite.

(ii) For $X \cong q^{n} \Theta(\pi)$, we take $P_{0}(X)=q^{n} P_{\Theta}(\pi)$. Now proceed by induction on the $\Theta$-filtration length of $X$. We may assume that there exists a short exact sequence $0 \rightarrow U \stackrel{\mathrm{i}}{\longrightarrow} X \stackrel{\mathrm{p}}{\longrightarrow} Y \rightarrow 0$ with non-trivial $U, Y \in \mathrm{Fil}(\Theta)$. By induction, there are epimorphisms $\varepsilon_{U}: P_{0}(U) \rightarrow U$ and $\varepsilon_{Y}: P_{0}(Y) \rightarrow Y$ with $P_{0}(U), P_{0}(Y)$ of the required form, and the kernels $U^{\prime}$ of $\varepsilon_{U}$ and $Y^{\prime}$ of $\varepsilon_{Y}$ in Fil $(\Theta)$. Since $\operatorname{Ext}^{1}\left(P_{0}(Y), U\right)=0$, there is $\alpha: P_{0}(Y) \rightarrow X$ with $\alpha \circ \mathrm{p}=\varepsilon_{Y}$. Then [ $\left.\varepsilon_{U} \circ \dot{i}, \alpha\right]:$ $P_{0}(U) \oplus P_{0}(Y) \rightarrow X$ is surjective, and its kernel is an extension of $U^{\prime}$ by $Y^{\prime}$.

A weakly $\mathscr{B}$-standardizing family $\Theta$ is called $\mathscr{B}$-standardizing if the following additional condition holds:

(Fr) For each $\pi, \sigma \in \Pi$, the $B_{\sigma}$-module $\operatorname{Hom}\left(P_{\Theta}(\pi), \Theta(\sigma)\right)$ is free finite rank.

A standard example is as follows. If $\mathbf{C}$ is a $\mathscr{B}$-highest weight category with poset $\Sigma$, and $\Pi \subseteq \Sigma$ is a finite saturated subset, then $\Delta:=\{\Delta(\pi) \mid \pi \in \Pi\}$ is a $\mathscr{B}$-standardizing family. Indeed, we have already observed in 98.1 that $\Delta$ is a weakly $\mathscr{B}$-standardizing family. Now (HWC) in the category $\mathbf{C}(\Pi)$ yields $(\mathrm{Fr})$.

The proof of the next theorem follows that of [18, Theorem 2]. 
Theorem 8.3. Let $\Theta$ be a (weakly) $\mathscr{B}$-standardizing family in a graded abelian $F$-linear category $\mathbf{C}$. Then there exists a (weakly) $\mathscr{B}$-quasihereditary algebra $H$, unique up to a graded Morita equivalence, such that the category $\operatorname{Fil}(\Theta)$ and the category $\mathrm{Fil}(\Delta)$ of graded $H$-modules with finite $\Delta$-filtrations are graded equivalent.

Proof. For each $\pi \in \Pi$, we have an object $P_{\Theta}(\pi) \in$ Fil $(\Theta)$ constructed in Lemma 8.2. Let $P:=\bigoplus_{\pi \in \Pi} P_{\Theta}(\pi)$ and $H:=\operatorname{End}(P)^{\mathrm{op}}$. Note by (End) and (FG) that $H$ is a left Noetherian Laurentian graded algebra. Consider the functor

$$
\mathcal{F}:=\operatorname{Hom}(P,-): \operatorname{Fil}(\Theta) \rightarrow H-\operatorname{Mod} .
$$

As $\operatorname{Ext}^{1}(P, X)=0$ for all $X \in \operatorname{Fil}(\Theta)$, the functor is exact on exact sequences $0 \rightarrow U \rightarrow X \rightarrow V$ with $U, V, X \in \mathrm{Fil}(\Theta)$. Set $P(\pi):=\mathcal{F}\left(P_{\Theta}(\pi)\right)$ and $\Delta(\pi):=$ $\mathcal{F}(\Theta(\pi))$ for all $\pi \in \Pi$. Note that $\mathcal{F}$ maps modules in $\operatorname{Fil}(\Theta)$ to the modules in $\operatorname{Fil}(\Delta)$. From now on we consider $\mathcal{F}$ as a functor from $\operatorname{Fil}(\Theta)$ to $\operatorname{Fil}(\Delta)$.

Claim 1. The functor $\mathcal{F}: \operatorname{Fil}(\Theta) \rightarrow \operatorname{Fil}(\Delta)$ is fully faithful.

To prove the claim, note first that it is true on finite direct sums of objects of the form $q^{n} P_{\Theta}(\pi)$. Let $X$ be an arbitrary object of Fil $(\Theta)$. By Lemma 8.2(ii), there is an exact sequence

$$
P_{1}(X) \stackrel{\delta_{X}}{\longrightarrow} P_{0}(X) \stackrel{\varepsilon_{X}}{\longrightarrow} X \rightarrow 0
$$

in $\mathbf{C}$ such that $X^{\prime}:=\operatorname{im} \delta_{X}$ and $X^{\prime \prime}=\operatorname{ker} \delta_{X}$ belong to Fil $(\Theta)$. Under $\mathcal{F}$, the exact sequence above goes to a projective presentation of $\mathcal{F}(X)$. Now assume that $X, Y \in \operatorname{Fil}(\Theta)$. Let $f: X \rightarrow Y$ be a map with $\mathcal{F}(f)=0$. There exist maps $f_{0}, f_{1}$ which make the following diagram commutative:

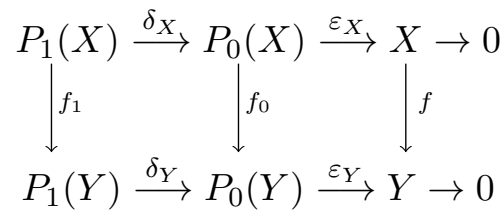

Since $F(f)=0$, there is a map $g^{\prime}: \mathcal{F}\left(P_{0}(X)\right) \rightarrow \mathcal{F}\left(P_{1}(Y)\right)$ such that $\mathcal{F}\left(\delta_{Y}\right) \circ g^{\prime}=$ $\mathcal{F}\left(f_{0}\right)$. However, $g^{\prime}=\mathcal{F}(g)$ for some $g: P_{0}(X) \rightarrow P_{1}(Y)$ such that $f_{0}=\delta_{Y} \circ g$. Hence $f \circ \varepsilon_{X}=\varepsilon_{Y} \circ \delta_{Y} \circ g=0$, and so $f=0$. We have proved that $\mathcal{F}$ is faithful. In order to prove that it is full, let $f^{\prime}: \mathcal{F}(X) \rightarrow \mathcal{F}(Y)$ be a morphism. We then obtain the maps $f_{0}^{\prime}$ and $f_{1}^{\prime}$ which make the following diagram commutative

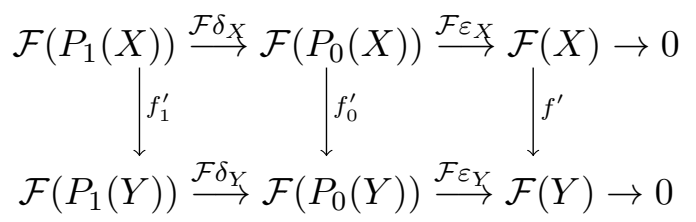

We can write $f_{0}^{\prime}=\mathcal{F}\left(f_{0}\right)$ and $f_{1}^{\prime}=\mathcal{F}\left(f_{1}\right)$, and we have $\delta_{Y} \circ f_{1}=f_{0} \circ \delta_{X}$. Since $\varepsilon_{Y} \circ f_{0} \circ \delta_{X}=0$, there is $f: X \rightarrow Y$ such that $\varepsilon_{Y} \circ f_{0}=f \circ \varepsilon_{X}$. Then

$$
\mathcal{F} f \circ \mathcal{F} \varepsilon_{X}=\mathcal{F} \varepsilon_{Y} \circ \mathcal{F} f_{0}=\mathcal{F} \varepsilon_{Y} \circ f_{0}^{\prime}=f^{\prime} \circ \mathcal{F} \varepsilon_{X} .
$$

As $\varepsilon_{X}$ is an epimorphism, we have $\mathcal{F} f=f^{\prime}$. This completes the proof of Claim 1.

To prove that $\mathcal{F}$ is equivalence, it remains to prove that any $M \in \operatorname{Fil}(\Delta)$ is isomorphic to $\mathcal{F}(X)$ for some $X \in \mathrm{Fil}(\Theta)$. We apply induction on the length 
of a $\Delta$-filtration of $M$, the result being clear for length 1 . Let $U \subseteq M$ be a submodule with $U \cong q^{n} \Delta(\pi)$ for some $n$ and $\pi$ and $M / U \in \operatorname{Fil}(\Delta)$. Let i : $q^{n} \Delta(\pi) \rightarrow M$ be a monomorphism with the image $U$ and $\mathrm{p}: M \rightarrow M / U$ be the projection. By induction $M / U \cong F(Y)$ for some $Y \in$ Fil $(\Theta)$. By Lemma 8.2(ii), there is $P_{0}(Y) \in \mathrm{Fil}(\Theta)$ which is a finite direct sum of modules of the form $q^{m} P_{\Theta}(\pi)$ and an epimorphism $\varepsilon_{Y}: P_{0}(Y) \rightarrow Y$ with $Y^{\prime}:=\operatorname{ker} \varepsilon_{Y} \in \mathrm{Fil}(\Theta)$. Let $\mathrm{u}: Y^{\prime} \rightarrow P_{0}(Y)$ be the inclusion map. Since $\mathcal{F}\left(P_{0}(Y)\right)$ is projective, there is a map a $: \mathcal{F}\left(P_{0}(Y)\right) \rightarrow M$ such that $\pi \circ$ a $=\mathcal{F}\left(\varepsilon_{Y}\right)$. Then

$$
[\mathrm{i}, \mathrm{a}]: q^{n} \Delta(\pi) \oplus \mathcal{F}\left(P_{0}(Y)\right) \rightarrow M
$$

is surjective, and its kernel is isomorphic to $\mathcal{F}\left(Y^{\prime}\right)$ with the kernel map of the form $[\mathrm{f}, \mathcal{F}(\mathrm{u})]: \mathcal{F}\left(Y^{\prime}\right) \rightarrow q^{n} \Delta(\pi) \oplus \mathcal{F}\left(P_{0}(Y)\right)$ for some map $\mathrm{f}: \mathcal{F}\left(Y^{\prime}\right) \rightarrow q^{n} \Delta(\pi)$. Since $\mathcal{F}\left(Y^{\prime}\right)$ and $q^{n}(\Delta(\pi))$ are images under $\mathcal{F}$, and $\mathcal{F}$ is full, there is a map $\mathrm{h}: Y^{\prime} \rightarrow q^{n} \Theta(\pi)$ with $\mathcal{F}(\mathrm{h})=\mathrm{f}$. As $\mathrm{u}$ is am monomorphism, we conclude that $[\mathrm{h}, \mathrm{u}]: Y^{\prime} \rightarrow q^{n} \Theta(\pi) \oplus P_{0}(Y)$ is also a monomorphism. Let $X:=\operatorname{coker}[\mathrm{h}, \mathrm{u}]$. Since $\mathrm{u}=[\mathrm{h}, \mathrm{u}] \circ\left[\begin{array}{c}0 \\ \mathrm{id}\end{array}\right]$, the cockerel $X$ maps onto the cockerel $Y$ of $\mathrm{u}$, say by $\mathrm{e}: X \rightarrow Y$, and ker $\mathrm{e}=q^{n} \Theta(\pi)$. We conclude that $X$ is an extension of $Y$ and $q^{n} \Theta(\pi)$. The exact sequence

$$
0 \rightarrow Y^{\prime} \stackrel{[\mathrm{h}, \mathrm{u}]}{\longrightarrow} q^{n} \Theta(\pi) \oplus P_{0}(Y) \longrightarrow X \rightarrow 0
$$

goes under $\mathcal{F}$ to an exact sequence because $Y^{\prime} \in \mathrm{Fil}(\Theta)$. So $\mathcal{F}(X)$ is isomorphic to the cockerel of $\mathcal{F}([\mathrm{h}, \mathrm{u}])=[\mathrm{f}, \mathcal{F}(\mathrm{u})]$, which is isomorphic to $M$.

We have now proved that $\mathcal{F}: \operatorname{Fil}(\Theta) \rightarrow \operatorname{Fil}(\Delta)$ is an equivalence. Claim 1 implies that $H$-mod satisfies the axioms (SC1), (SC2), and (HWC) (resp. (FGen) if we start with weakly $\mathscr{B}$-standardizing family). By Theorem 6.7, we conclude that $H$ is (weakly) $\mathscr{B}$-quasihereditary with standard modules $\Delta(\pi)$.

\section{9. $\mathscr{B}$-QUASIHEREDITARY ALGEBRAS WITH INVOLUTION}

9.1. Balanced involution. Let $H$ be a left Noetherian Laurentian algebra. Suppose that $H$ has a homogeneous antiinvolution $\tau: h \mapsto h^{\prime}$. This allows us to consider, for any left $H$-module $V$, the right $H$-module $V^{\tau}$ with $v h:=h^{\prime} v$ for all $v \in V, h \in H$. Moreover, given an $H$-module $V$ with finite dimensional graded components $V_{n}$ we define its graded dual $V^{\circledast}$ as a left $H$-module, which as a graded vector space has $V_{n}^{\circledast}:=V_{-n}^{*}$ for all $n \in \mathbb{Z}$, and the action $h f(v)=f\left(h^{\prime} v\right)$ for $f \in V^{\circledast}, v \in V$ and $h \in H$. Note that $\left(q^{n} V\right)^{\circledast} \cong q^{-n} V^{\circledast}$ and $\operatorname{dim}_{q} V^{\circledast}=\operatorname{dim}_{q^{-1}} V$.

Recall that we have chosen a complete irredundant set $\{L(\pi) \mid \pi \in \Pi\}$ of irreducible $H$-modules up to isomorphism and degree shift. The homogeneous antiinvolution $h \mapsto h^{\prime}$ of $H$ is called balanced if for every $\pi \in \Pi$ we have that $L(\pi)^{\circledast} \cong q^{n} L(\pi)$ for some even integer $n$. In that case, we can redefine $L(\pi):=$ $q^{n / 2} L(\pi)$ to achieve that

$$
L(\pi)^{\circledast} \cong L(\pi) \quad(\pi \in \Pi) .
$$

Throughout the section, we assume that the algebra $H$ possesses a balanced antiinvolution $\tau$, and the irreducible modules $L(\pi)$ are chosen so that (9.1) holds. Then $P(\pi)^{\circledast}$ is the injective hull of $L(\pi)$, and for a $\mathscr{B}$-stratified $H$, it follows from definitions of $₫ 4.1$ and 97.1 that $\bar{\Delta}(\pi)^{\circledast} \cong \bar{\nabla}(\pi)$ for all $\pi \in \Pi$. In particular, 
Theorem 7.6 implies

$$
(P(\pi): \Delta(\sigma))_{q}=[\bar{\Delta}(\sigma): L(\pi)]_{q} .
$$

9.2. Affine heredity and affine cell ideals. Koenig and Xi [35] have studied the notion of an affine cellular algebra. We now define its graded version and show, by analogy with a well-known classical fact, that affine quasihereditary algebras with involution are graded affine cellular.

Let, as usual, $H$ be a left Noetherian Laurentian algebra, and $\tau$ be a homogeneous antiinvolution on $H$. In the following definition, which is a graded version of [35. Definition 2.1], we do not need to assume that $\tau$ is balanced. Recall that we assume that all algebras, ideals, vector spaces, and so on are assumed graded.

Definition 9.3. An ideal $J$ of $H$ is (graded) affine cell if the following data are given and the following conditions are satisfied:

(Inv) $\tau(J)=J$.

(Stand) There exist a finite dimensional vector space $V$, an affine algebra $B$ with a homogeneous involution $\iota$, and an $(H, B)$-bimodule structure on $\Delta:=V \otimes B$, where the right $B$-module structure is induced by that of the regular module $B_{B}$.

(Sym) Let $\Delta^{\prime}:=B \otimes V$ be the $(B, H)$-bimodule with the left $B$-module structure induced by that of the regular module ${ }_{B} B$ and right $H$-module structure defined by

$$
(b \otimes v) a=\mathbf{s}(\tau(a)(v \otimes b)),
$$

where $\mathrm{s}: V \otimes B \rightarrow B \otimes V, v \otimes b \mapsto b \otimes v$; then there is an $(H, H)$ bimodule isomorphism $\mu: J \rightarrow \Delta \otimes_{B} \Delta^{\prime}$, such that the following diagram commutes:

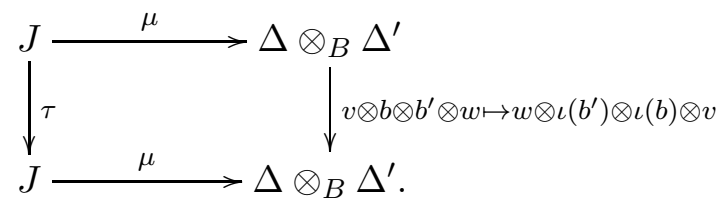

The algebra $H$ is called (graded) affine cellular if there is a vector space decomposition $H=J_{1}^{\prime} \oplus J_{2}^{\prime} \oplus \cdots \oplus J_{n}^{\prime}$ with $\tau\left(J_{l}^{\prime}\right)=J_{l}^{\prime}$ for all $1 \leq l \leq n$, such that, setting $J_{m}:=\bigoplus_{l=1}^{m} J_{l}^{\prime}$, we obtain an ideal filtration

$$
0=J_{0} \subset J_{1} \subset J_{2} \subset \cdots \subset J_{n}=H,
$$

and each $J_{m} / J_{m-1}$ is an affine cell ideal of $H / J_{m-1}$.

Remark 9.6. If the characteristic $p$ of the ground field is not 2 , then the final condition in the definition above can be relaxed. We could just require that there is an ideal filtration $0=J_{0} \subset J_{1} \subset J_{2} \subset \cdots \subset J_{n}=H$, such that for each $m=1, \ldots, n$, we have $\tau\left(J_{m}\right)=J_{m}$ and $J_{m} / J_{m-1}$ is an affine cell ideal of $H / J_{m-1}$. Indeed, if $p \neq 2$ then $\tau$ is a semisimple linear transformation. So, given a $\tau$-invariant subideal $I_{m-1}$ in a $\tau$-invariant ideal $I_{m}$, we can always find a $\tau$-invariant subspace $I_{m}^{\prime}$ inside $I_{m}$ such that $I_{m-1} \oplus I_{m}^{\prime}=I_{m}$.

The following lemma is inspired by [13, (2.2)]. Recall that 'affine quasihereditary' means ' $\mathscr{B}$-quaihereditary' for the case where $\mathscr{B}$ is the class of affine algebras. 
Lemma 9.7. Let $H$ be an affine quasihereditary algebra with a balanced involution $\tau: H \rightarrow H, h \mapsto h^{\prime}$ and affine heredity chain $0=J_{0} \subset J_{1} \subset J_{2} \subset \cdots \subset$ $J_{n}=H$. Then the ideals $J_{m}$ are $\tau$-invariant for all $m=1, \ldots, n$.

Proof. It suffices to prove that $J_{1}$ is $\tau$-invariant. By Lemma 6.5, we have that $J_{1}=H e H$ for some idempotent $e \in H$. For any $\pi \in \Pi$, we have $L(\pi)^{\circledast} \cong L(\pi)$, whence $e L(\pi) \neq 0$ if and only if $e^{\prime} L(\pi) \neq 0$. Hence the projective module $H e$ has the projective indecomposable module $P(\pi)$ as a summand if and only if $H e^{\prime}$ does. It now follows that $H e H=H e^{\prime} H$.

In the situation of the lemma, we say that the balanced involution $\tau$ is split if there is a vector space decomposition $H=J_{1}^{\prime} \oplus J_{2}^{\prime} \oplus \cdots \oplus J_{n}^{\prime}$ with $\tau\left(J_{l}^{\prime}\right)=J_{l}^{\prime}$ for $1 \leq l \leq n$, and such that $J_{m}:=\bigoplus_{l=1}^{m} J_{l}^{\prime}$. The argument of Remark 9.6 shows that every balanced involution is automatically split unless the characteristic of the ground field is 2 .

Proposition 9.8. Let $H$ be an affine quasihereditary algebra with a balanced split involution $\tau$. Then $H$ is an affine cellular algebra.

Proof. Let $J$ be an affine heredity ideal in $H$. It suffices to show that it is an affine cell ideal. By Lemma 6.5, we have that $J=H e H$ for some idempotent $e \in H$. By Lemma 9.7, we know that $J$ is $\tau$-invariant.

To check the property (Stand), recall from Theorem 6.7(ii), that there exists $m(q) \in \mathbb{Z}\left[q, q^{-1}\right]$ and a maximal element $\pi \in \Pi$ such that $J \cong m(q) P(\pi)$ and $P(\pi)=\Delta(\pi)$. Moreover, by Lemma 6.6, we may assume that $H e \simeq P(\pi)$, and $B_{\pi} \cong e H e$. Now, $P(\pi)=\Delta(\pi)$ is a free right module over $B_{\pi}$ by the property (HI2). Now (Stand) with $V=\bar{\Delta}(\pi)$ and $B=B_{\pi}$ follows from Proposition 5.7 .

To check (Sym), note by Lemma 6.6, that the natural map $H e \otimes_{e H e} e H \rightarrow J$ is an isomorphism. In the previous paragraph, we have identified $H e$ with $\Delta=$ $V \otimes B$ in (Stand), where $V \cong \bar{\Delta}(\pi), B=B_{\pi}=e H e$. This can be restated as follows: there exist linearly independent elements $v_{1} e, \ldots, v_{n} e \in H e$ such that every element he $\in H e$ can be written in the from

$$
h e=\sum_{i} v_{i} e \beta_{i}(h) e \quad\left(\beta_{i}(h) \in H\right),
$$

and, denoting $V=\operatorname{span}\left(v_{1} e, \ldots, v_{n} e\right)$, the map

$$
H e \rightarrow V \otimes e H e=\Delta, h e \mapsto \sum_{i} v_{i} e \otimes e \beta_{i}(h) e
$$

is an isomorphism of vector spaces.

Now, we have that $P(\pi) \simeq H e \simeq H e^{\prime}$. Hence there exist $u_{1}, u_{2} \in H$ with $e u_{1} e^{\prime} u_{2} e=e$ and $e^{\prime} u_{2} e u_{1} e^{\prime}=e^{\prime}$, such that the isomorphism $H e \stackrel{\sim}{\longrightarrow} H e^{\prime}$ is given by the right multiplication with $e u_{1} e^{\prime}$ and the inverse isomorphism is given by right multiplication with $e^{\prime} u_{2} e$. Now, the left multiplication with $e^{\prime} u_{2} e$ also gives an isomorphism $e H \stackrel{\sim}{\longrightarrow} e^{\prime} H$ as right $H$-modules. Moreover, considering $e^{\prime} H$ as a left $e H e$-module via the action

$$
(e \beta e) \cdot e^{\prime} h:=e^{\prime} u_{2} e \beta e u_{1} e^{\prime} h \quad(\beta, h \in H),
$$

the isomorphism above is an isomorphism of $(e H e, H)$-bimodules. It is easy to see that the $(e H e, H)$-bimodule $e^{\prime} H$ we have just defined is isomorphic to the $(e H e, H)$-bimodule $\Delta^{\prime}=e H e \otimes V$ defined from $\Delta=H e=V \otimes e H e$ using the rule (9.4). 
Composing the bimodule isomorphisms $e H \stackrel{\sim}{\longrightarrow} e H^{\prime} \stackrel{\sim}{\longrightarrow} \Delta^{\prime}$ described in the previous paragraph, we get an isomorphism

$$
\begin{aligned}
\mu: J= & H e \otimes_{e H e} e H \stackrel{\sim}{\longrightarrow} \Delta \otimes_{e H e} \Delta^{\prime}, \\
& h_{1} e \otimes e h_{2} \mapsto \sum_{i, j} v_{i} e \otimes e \beta_{i}\left(h_{1}\right) e \otimes e \beta_{j}\left(h_{2}^{\prime} e^{\prime} u_{2}^{\prime}\right) e \otimes v_{j} e,
\end{aligned}
$$

using the notation of (9.9).

Finally, we check the commutativity of (9.5) with $\iota=$ id, i.e. that

$$
\mu\left(\tau\left(h_{1} e h_{2}\right)\right)=\sum_{i, j} v_{j} e \otimes e \beta_{j}\left(h_{2}^{\prime} e^{\prime} u_{2}^{\prime}\right) e \otimes e \beta_{i}\left(h_{1}\right) e \otimes v_{i} e
$$

for $h_{1}, h_{2} \in H$. Note that

$$
\mu\left(\tau\left(h_{1} e h_{2}\right)\right)=\mu\left(h_{2}^{\prime} e^{\prime} h_{1}^{\prime}\right)=\mu\left(h_{2}^{\prime} e^{\prime} u_{2} e u_{1} e^{\prime} h_{1}^{\prime}\right),
$$

and so (9.10) follows from the definition of $\mu$, since $h_{1} e u_{1}^{\prime} e^{\prime} u_{2}^{\prime} e=h_{1} e$.

\section{EXAmples}

Let $\mathscr{P}$ be the class of graded polynomial algebras, cf. $\$ 5.2$.

10.1. KLR algebras. Khovanov-Lauda [27, 28] and Rouquier [45] have introduced a family of algebras $R_{\alpha}(\mathrm{C})$ labeled by a generalized Cartan matrix $\mathrm{C}$ and an element $\alpha$ of the non-negative part of the corresponding root lattice. These algebras proved to be of fundamental importance.

Assume that $\mathrm{C}$ is of finite type, i.e. $\mathrm{C}$ is of types $\mathrm{A}_{1}, \mathrm{~B}_{1}, \ldots, \mathrm{E}_{8}$. It is proved in [7] using homological and representation theoretic methods that $R_{\alpha}(\mathrm{C})$-mod is a polynomial highest weight category, i.e. $\mathscr{P}$-highest weight category. On the other hand, it is proved in [32, see also [33 for type $\mathrm{A}_{1}$ only, that $R_{\alpha}(\mathrm{C})$ is $\mathscr{P}$-quasihereditary by constructing an explicit $\mathscr{P}$-heredity chain in it. Of course, this illustrates Theorem 6.7. Moreover, in view of Corollary 5.25, the algebras $R_{\alpha}(\mathrm{C})$ have finite global dimension, this result was established in $[\mathbf{2 4}, \mathbf{2 5}, 40$, see also [7, Appendix].

The case where $\mathrm{C}$ is not of finite type is open. It seems that a more general notion of a $\mathscr{B}$-stratified algebra is needed.

10.2. Kato's geometric extension algebras. Given a connected algebraic group $G$ acting on a variety $X$ over $\mathbb{C}$ with finitely many orbits $\left\{\mathcal{O}_{\lambda}\right\}_{\lambda \in \Lambda}$ and assuming three further natural geometric conditions, S. Kato [24] defines the corresponding geometric extension algebra

$$
A=A_{(G, X)}:=\bigoplus_{\lambda, \mu} \operatorname{Ext}_{D_{G}^{b}(X)}^{\bullet}\left(L_{\lambda} \otimes \mathrm{IC}_{\lambda}\left[\operatorname{dim} \mathcal{O}_{\lambda}\right], L_{\mu} \otimes \mathrm{IC}_{\mu}\left[\operatorname{dim} \mathcal{O}_{\lambda}\right]\right),
$$

where $L_{\lambda}$ is a self-dual non-zero graded vector space for each $\lambda \in \Lambda$. As explained in [24, geometric extension algebras arising from affine Hecke algebras of types $A$ and $B C$, the Khovanov-Lauda-Rouquier algebras (over $\mathbb{C}$ ) of finite ADE types, the quiver Schur algebras, and the algebra which governs the BGG category all fit into this class.

It is proved in 24 that the category $A$-mod is a $\mathscr{P}$-highest weight category, see [24, Theorem C, Lemma 1.3, proof of Corollary 3.3]. So $A$ is $\mathscr{P}$-quaihereditary. 
10.3. Graded representations of current algebras. Here we follow [10], see also [1], 5], 6], 9] and references therein. Let $\mathfrak{C} \mathfrak{g}$ be the current algebra corresponding to the arbitrary indecomposable affine Lie algebra $\mathfrak{g}$, see [10, (2.3)]. As explained in [10, Remark 2.1], outside of the type $A_{2 \ell}^{(2)}$, this is the usual (possibly twisted) current Lie algebra of a finite dimensional simple Lie algebra. This Lie algebra has a natural $\mathbb{Z}_{\geq 0}$-grading [10, $\left.\S 2.1\right]$. Let $\mathbf{C}$ be the category of finitely generated graded $\mathfrak{C} \mathfrak{g}$-modules. It is easy to check, using the description of the projective modules in [10], that $\mathbf{C}$ is a graded Noetherian Laurentian category, with $q$ being the degree shift by $a_{0}$ (we have $a_{0}=1$, unless we are in type $A_{2 \ell}^{(2)}$, in which case $a_{0}=2$ ).

Note that the category $\mathbf{C}$ is smaller than the category $\mathfrak{c} \mathfrak{g} \mathfrak{F}^{\mathbb{Z}}$ considered in [10, §2.3], but the following important classes of modules considered in [10] all lie in this smaller category. The irreducible modules in $\mathbf{C}$ are $q^{k} L(\lambda)$ denoted $\pi^{*} \stackrel{\circ}{V}(\lambda+k \delta)$ in [10, $\left.\S 2.4\right]$, and labeled by the dominant weights $\lambda \in \Pi:=\stackrel{\circ}{P}$ of the corresponding finite dimensional simple Lie algebra and $k \in \mathbb{Z}$. We also have the projective covers $q^{k} P(\lambda)$ of $q^{k} L(\lambda)$, denoted $P(\lambda+k \delta)$ in [10, $\left.\S 2.5\right]$ and the Weyl modules $W(\lambda+k \delta)$.

Using the standard dominance order on the set $\Pi$ of dominant weights, we have, in view of [10, Proposition 2.4(i)], that $W(\lambda+k \delta)=q^{k} \Delta(\lambda)$. Moreover, it follows from [10, Theorem 2.5] that $B_{\lambda}:=\operatorname{End}_{\mathfrak{C g}}(\Delta(\lambda))^{\mathrm{op}}$ is a graded polynomial algebra (denoted $\mathbf{A}_{\lambda}$ in $[\mathbf{1 0}]$ ), and $\Delta(\lambda)$ is a finitely rank free $B_{\lambda}$-module, cf. [10, Corollary 2.10]. Finally, it follows from [10, Theorem 4.8] that $K(\lambda)$ has a $\Delta$-filtration with factors $\simeq \Delta(\mu)$ for $\mu>\lambda$, cf. the property (SC1) in Definition 5.2. It follows that $\mathbf{C}$ is a $\mathscr{P}$-highest weight category.

The local Weyl modules $W_{l o c}(\lambda+k \delta)$ are defined in [10, $\left.\$ 2.8\right]$. Comparing this definition with Proposition 5.6(iii), we conclude that $W_{l o c}(\lambda+k \delta) \cong q^{k} \bar{\Delta}(\lambda)$. Moreover, using the duality '*' from [10, $\S 2.3]$, it is easy to see that $\bar{\nabla}(\lambda)$ has the same composition multiplicities as $\bar{\Delta}(\lambda)$. Now [10, Theorem 4.8] is a special case of Theorem 7.6 .

In view of Theorem 6.7, choosing a finite saturated subset of weights $\Sigma \subset \Pi$, we get the subcategory $\mathbf{C}(\Sigma)$ is equivalent to the category of finitely generated graded modules over some $\mathscr{P}$-quasihereditary algebra $H_{\Sigma}$. It would be interesting to find a more explicit description of this algebra and its connection to the $\mathscr{P}$ quasihereditary algebras described in other examples of this section.

It would also be interesting to try to incorporate representation theory of the more general equivariant map algebras in place of (twisted) current algebras, cf. [21].

Question. Let $\mathfrak{g}$ be a positively graded Lie algebra with reductive zero degree component $\mathfrak{g}_{0}$. When is the category of graded finitely generated $\mathfrak{g}$-modules a (weakly) $\mathscr{B}$-highest weight category.

This seems to be especially interesting for the Lie algebras of formal vector fields on $\mathbb{C}^{n}$ vanishing at the origin and the Lie subalgebra of Hamiltonian vector fields with trivial constant and linear terms, cf. [26. Examples 1.2.4, 1.2.5] and [26. Conjecture 4.86]. It is also interesting to consider the modular Lie algebras of Cartan type. Some relevant results have already been obtained in [36, 37.

10.4. Graded representation theory of $\mathbb{C} W \ltimes \mathbb{C}\left[\mathfrak{h}^{*}\right]$. Let $W$ be a real reflection group and $\mathfrak{h}$ be its reflection representation. Then the algebra $A_{W}:=$ 
$\mathbb{C} W \ltimes \mathbb{C}\left[\mathfrak{h}^{*}\right]$ is graded so that $\operatorname{deg}(w)=0$ for all $w \in W$ and $\operatorname{deg}(x)=2$ for $x \in \mathfrak{h}^{*}$. It is essentially shown in $\left[23\right.$ that the category $A_{W}$-mod of finitey generated graded $A_{W}$-modules is $\mathscr{P}$-highest weight. In particular, by Theorem 6.7. the algebra $A_{W}$ is $\mathscr{P}$-quasihereditary. The role of proper standard modules is played by the elements of so-called Kostka system introduced in [23.

10.5. Other possible examples. We conjecture that the (graded) affine Schur algebras are $\mathscr{P}_{\text {-quasihereditary. A partial confirmation can be found in 47. }}$.

The categories of Khovanov and Sazdanovich [29] used to category Hermite polynomials seem to be $\mathscr{B}$-highest weight for $\mathscr{B}$ the class of nilCoxeter algebras.

The odd nilHecke algebras [19] and quiver Hecke(-Clifford) superalgebras of 22 should satisfy the (super analogue of) the axioms of $\mathscr{B}$-quasihereditary for a class $\mathscr{B}$ of algebras which are built out of polynomial and Clifford algebras.

Finally, according to [4, Verma modules over Cherednik algebras exhibit some features, which make them candidates for the standard modules in an appropriate $\mathscr{B}$-highest weight category.

\section{REFERENCES}

[1] H.H. Andersen, J.C. Jantzen and W. Soergel, Representations of quantum groups at a $p$-th root of unity and of semisimple groups in characteristic $p$ : independence of $p$, Astérisque 220(1994).

[2] K.S. Brown, Homological criteria for finiteness, Comment. Math. Helv. 50(1975), 129135 .

[3] H. Bass, Algebraic K-Theory, W.A. Benjamin, Inc., New York, 1968.

[4] G. Bellamy, Endomorphisms of Verma modules for rational Cherednik algebras, arXiv: 1312.7524 .

[5] M. Bennett, V. Chari, and N. Manning, BGG reciprocity for current algebras, Adv. Math. 231 (2012), 276-305.

[6] M. Bennet, A. Berenstein, V. Chari, A. Khoroshkin, and S. Loktev, Macdonald polynomials and BGG reciprocity for current algebras, Selecta Math. (to appear); arXiv: 1207. 2446 .

[7] J. Brundan, A. Kleshchev, and P.J. McNamara, Homological properties of finite type Khovanov-Lauda-Rouquier algebras, Duke Math. J. 163 (2014), 1353-1404; arXiv: 1210.6900 .

[8] J. Brundan, I. Losev and B. Webster, Tensor product categorifications and the super Kazhdan-Lusztig conjecture, arXiv:1310.0349.

[9] V. Chari, G. Fourier, and T. Khandai, A categorical approach to Weyl modules, Transform. Groups 15 (2010), 517-549.

[10] V. Chari and B. Ion, BGG reciprocity for current algebras, arXiv: 1307.1440.

[11] V. Chari and A. Pressley, Weyl modules for classical and quantum affine algebras, Represent. Theory 5 (2001), 191-223.

[12] E. Cline, B. Parshall and L. Scott, Finite dimensional algebras and highest weight categories, J. Reine Angew. Math. 391 (1988), 85-99.

[13] E. Cline, B. Parshall and L. Scott, Duality in highest weight categories, pp. 7-22 in Classical Groups and Related Topics (Beijing, 1987), Contemp. Math. 82, Amer. Math. Soc., Providence, RI, 1989.

[14] E. Cline, B. Parshall and L. Scott, Stratifying endomorphism Algebras, Memoirs AMS 124 (1996), Vol. 124.

[15] S. Dăscălescu, Graded semiperfect rings Bull. Math. de le Soc. Math. Roum. 36 (1992), 247-255.

[16] V. Dlab, Properly stratified algebras, C. R. Acad. Sci. Paris, 331 (2000), Sér. I, 191-196.

[17] V. Dlab and C.M. Ringel, Quasi-hereditary algebras, Illinois J. Math. 33 (1989), 280291. 
[18] V. Dlab and C.M. Ringel, The module theoretic approach to quasi-hereditary algebras, pp. 200-224 in Representations of Algebras and Related Topics, H. Tachikawa and S. Brenner editors, LMS Lecture Notes Series 168, CUP, 1992.

[19] A. Ellis, M. Khovanov, and A. Lauda, The odd nilHecke algebra and its diagrammatics, arXiv: 1111.1320.

[20] S. Donkin, The q-Schur algebra, CUP, 1998.

[21] G. Fourier, N. Manning, and A. Savage, Global Weyl modules for equivariant map algebras, arXiv: 1303.4437.

[22] S.-J. Kang, M. Kashiwara, S. Tsuchioka, Quiver Hecke superalgebras, arXiv:1107.1039.

[23] S. Kato, A homological study of Green polynomials, ArXiv:1111.4640.

[24] S. Kato, An algebraic study of extension algebras, ArXiv: 1207.4640v4.

[25] S. Kato, PBW bases and KLR algebras, Duke Math. J. 163 (2014), 619-663.

[26] A. Khoroshkin, Highest weight categories and Macdonald polynomials, ArXiv: 1312.7053.

[27] M. Khovanov and A. Lauda, A diagrammatic approach to categorification of quantum groups I, Represent. Theory 13 (2009), 309-347.

[28] M. Khovanov and A. Lauda, A diagrammatic approach to categorification of quantum groups II, Trans. Amer. Math. Soc., 363 (2011), 2685-2700.

[29] M. Khovanov and R. Sazdanovich, Categorification of the orthogonal polynomials, work in progress.

[30] A. Kleshchev, Cuspidal systems for affine Khovanov-Lauda-Rouquier algebras, Math. Z., 276 (2014), 691-726.

[31] A. Kleshchev, Representation theory and cohomology of Khovanov-Lauda-Rouquier algebras, Conference Proceedings of the program 'Modular Representation Theory of Finite and p-adic Groups' at the National University of Singapore, to appear; arXiv:1401.6151.

[32] A. Kleshchev and J. Loubert, Affine Cellularity of Khovanov-Lauda-Rouquier algebras of finite type, arXiv: 1310.4467.

[33] A. Kleshchev, J. Loubert, and V. Miemietz, Affine Cellularity of Khovanov-LaudaRouquier algebras in type A, J. Lond. Math. Soc., to appear; arXiv:1210.6542.

[34] A. Kleshchev and R. Muth, Imaginary Schur-Weyl duality, arXiv:1312.6104.

[35] S. Koenig, and C. Xi, Affine cellular algebras, Adv. Math. 229 (2012), 139-182.

[36] Z. Lin and D. Nakano, Representations of Hopf algebras arising from Lie algebras of Cartan type, J. Algebra 189 (1997) 529-567.

[37] Z. Lin and D. Nakano, Good filtrations for representations of Lie algebras of Cartan type, J. Pure Appl. Alg. 127 (1998) 231-256.

[38] I. Losev and B. Webster, On uniqueness of tensor products of irreducible categorifications, arXiv: 1303.4617v2.

[39] V. Mazorchuk, Koszul duality for stratified algebras II. Standardly stratified algebras, J. Aust. Math. Soc. 89 (2010), 23-49.

[40] P. McNamara, Finite dimensional representations of Khovanov-Lauda-Rouquier algebras I: finite type, J. Reine Angew. Math., to appear; arXiv:1207.5860.

[41] B. Mitchell, Theory of Categories, Academic Press, 1965.

[42] M. Mori, A cellular approach to the Hecke-Clifford superalgebra, arXiv: 1401.1722.

[43] C. Năstăsescu and F. Van Oystaeyen, Methods of Graded Rings, Springer, 2004.

[44] J.J. Rotman, An Introduction to Homological Algebra, Second Edition, Springer, 2009.

[45] R. Rouquier, 2-Kac-Moody algebras; arXiv:0812.5023.

[46] P. Tingley and B. Webster, Mirkovic-Vilonen polytopes and Khovanov-Lauda-Rouquier algebras, arXiv:1210.6921.

[47] G. Yang, Affine cellularity of $S_{\Delta}(2,2)$; arXiv:1402.2715.

Department of Mathematics, University of Oregon, Eugene, OR 97403, USA

E-mail address: klesh@uoregon.edu 